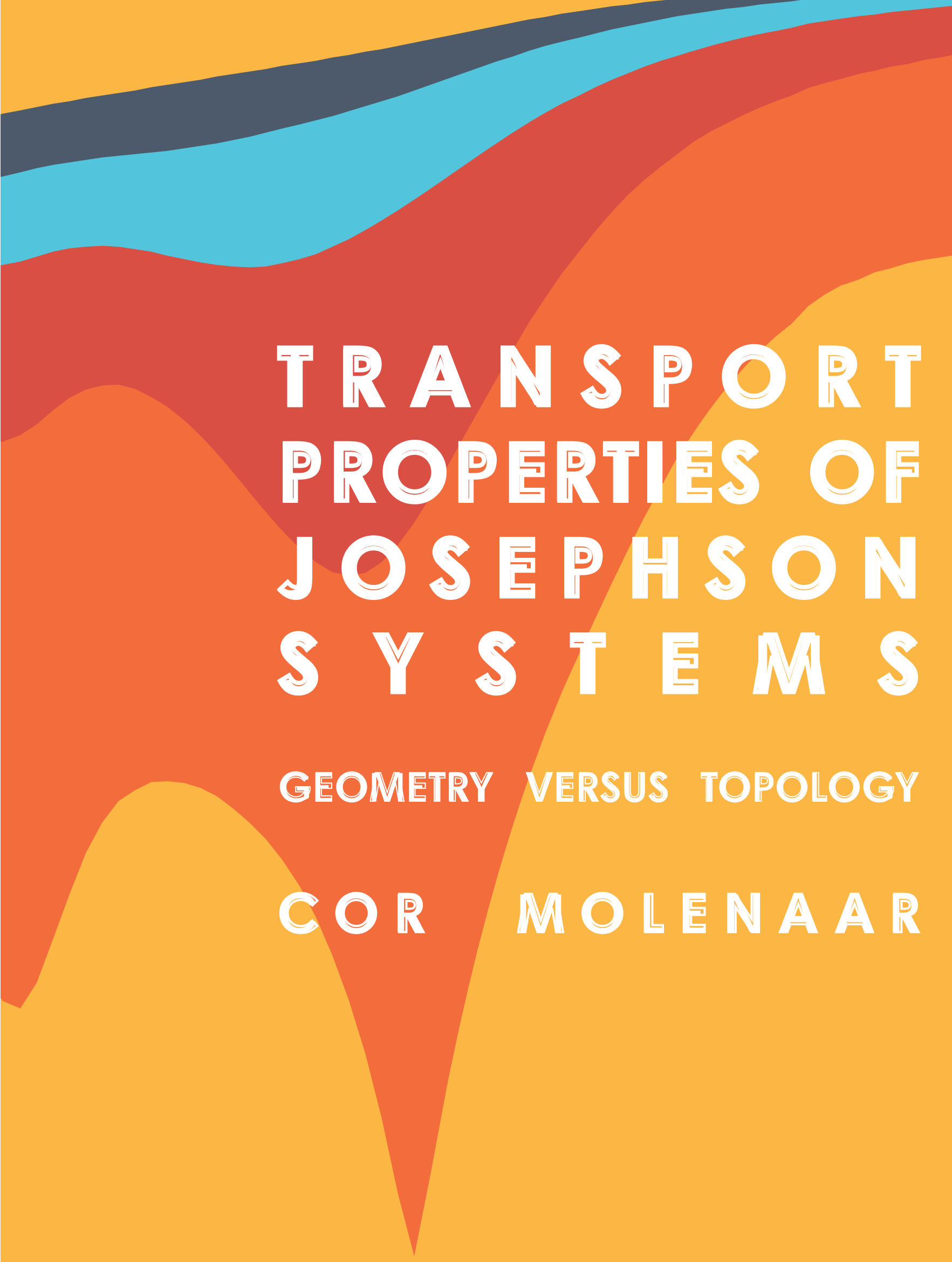




\section{TRANSPORT PROPERTIES OF JOSEPHSON SYSTEMS GEOMETRY VERSUS TOPOLOGY}




\section{Ph.D. committee}

Chairman

prof. dr. ir. H. Hilgenkamp University of Twente

Secretary

prof. dr. ir. H. Hilgenkamp University of Twente

Supervisors

prof. dr. ir. A. Brinkman University of Twente

prof. dr. ir. H. Hilgenkamp University of Twente

Members

prof. dr. E. Hankiewicz

prof. dr. ir. T. M. Klapwijk

University of Würzburg

dr. A. de Visser

Delft University of Technology

dr. ir. B. ten Haken

University of Amsterdam

prof. dr. ir. W. G. van der Wiel

University of Twente

University of Twente

\section{Cover}

The cover illustrates the magnetoresistance of an array of niobium islands coupled via a gold substrate, described in chapter 7 . Cover design by Nelliene Molenaar.

The research described in this thesis was performed in the Faculty of Science and Technology and the MESA ${ }^{+}$Institute for Nanotechnology at the University of Twente. The work was financially supported by the Netherlands Organization for Scientific Research (NWO) and the Dutch Foundation for Fundamental Research on Matter (FOM).

Transport properties of Josephson systems, Geometry versus Topology

Ph.D. Thesis, University of Twente

Printed by: Gildeprint

ISBN 978-90-365-3703-2

(C) C. G. Molenaar, 2014 


\section{TRANSPORT PROPERTIES OF JOSEPHSON SYSTEMS \\ GEOMETRY VERSUS TOPOLOGY}

\section{PROEFSCHRIFT}

ter verkrijging van

de graad van doctor aan de Universiteit Twente, op gezag van de rector magnificus, prof. dr. H. Brinksma

volgens besluit van het College voor Promoties in het openbaar te verdedigen

op vrijdag 5 september 2014 om 14:45 uur

door

Cornelis Germent Molenaar

geboren op 26 februari 1985

te Leiden 
Dit proefschrift is goedgekeurd door de promotors:

prof. dr. ir. A. Brinkman

prof. dr. ir. H. Hilgenkamp 


\section{CONTENTS}

I INTRODUCTION I

1.1 Superconductivity I

1.2 Topological insulators 2

1.3 Josephson junction arrays 3

2 INTRODUCTION TO BISMUTH BASED TOPOLOGICAL INSULATOR - SUPERCONDUCTOR SYSTEMS 7

2.1 Introduction 7

2.2 Topological insulators with proximity induced superconductivity 9

2.2.1 Supercurrents through TI structures 9

2.2.2 Current-phase relationship 11

2.2.3 dc SQUIDs with unconventional current phase relationships $\quad 14$

3 GEOMETRIC DEPENDENCE OF NB-BI $2 \mathrm{TE}_{3}-\mathrm{NB}$ TOPOLOGICAL JOSEPHSON JUNCTION TRANSPORT PARAMETERS 23

3.1 Introduction 23

3.2 Expected Majorana related modifications of the critical current modulation by magnetic field and microwaves 24

3.3 Sample layout and fabrication 25

3.4 Measured scaling of transport parameters 27

3.4.1 Junction overview 27

3.4.2 Scaling of $I_{C}$ and $R_{N} \quad 28$

3.4.3 Periodicity in $\Phi_{0} \quad 31$

3.5 Conclusion 33

4 JOSEPHSON SUPERCURRENT IN A TOPOLOGICAL INSULATOR WITHOUT A BULK SHUNT 37

4.1 Transport properties of exfoliated $\mathrm{Bi}_{1.5} \mathrm{Sb}_{0.5} \mathrm{Te}_{1.7^{-}}$ $\mathrm{Se}_{1.3}$ flakes 38

4.2 Junction fabrication 40

4.3 Results 42

4.3.I Temperature dependence of the critical current 43

4.3.2 Critical current as a function of magnetic field 45

4.4 Discussion 47

5 EXPERIMENTAL REALISATION OF TOPOLOGICAL INSULATOR SQUIDS 53 
5.1 Introduction 53

5.2 Device fabrication 55

$5 \cdot 3$ Characterisation 55

5.4 Analysis 60

5.5 Conclusions 60

6 OPTIMISING THE MAJORANA CHARACTER OF SQUIDS WITH TOPOLOGICALLY NON-TRIVIAL BARRIERS 63

6.1 Introduction 63

6.2 Model 64

6.2.1 Fluxoid quantisation in topologically (non)trivial rings 64

6.2.2 SQUID characteristics in the superconducting and voltage state 67

6.3 Results 67

6.3.1 dc SQUIDs composed of trivial and nontrivial elements $\quad 68$

6.3.2 Topologically non-trivial SQUIDs 69

6.3.3 The voltage state 73

6.4 Applications to topologically non-trivial systems 73

6.5 Conclusions 74

7 CRITICAL BEHAVIOUR AT THE DYNAMIC MOTT TRANSITION 77

7.1 Introduction 77

7.2 Mott phenomena and transition $\quad 78$

7.3 Sample design and fabrication $\quad 79$

7.4 Device characterisation 81

$7 \cdot 5$ Scaling analysis 84

7.6 Conclusion 88

8 DISORDERED PROXIMITY COUPLED ARRAYS 93

8.1 Background 93

8.2 Superconducting transition temperature 95

8.3 Behaviour in magnetic field 99

8.4 Cluster sizes in random arrays 100

8.5 Conclusion IOI

SUMMARY 104

SAMENVATTING 108

DANKWOORD 112 


\section{INTRODUCTION}

This chapter introduces the key concepts of the research presented in this thesis: the interplay between superconductivity and topological insulators, and between superconductivity and constraints due to patterned structures. Special attention is paid to the influence of geometry and the search for topological effects in experiments. A short overview of the subsequent chapters is also given.

\section{I.I SUPERCONDUCTIVITY}

Superconductivity is the result of the collective behaviour of electrons, distinguishing it from the behaviour of electrons in metals. This collective behaviour results in a macroscopic quantum state, and is the result of an attractive force between electrons $[1,2]$. These electrons would otherwise experience a Coulomb repulsion, but an attractive force allows two electrons to form a bosonic Cooper pair [3]. The formation of these bosons results in a new ground state, where all Cooper pairs occupy the same energy level. So, all Cooper pairs in a material occupy the same macroscopic quantum ground state which can be described using a wave function with a complex phase, $\Psi$ $\Delta \exp (i \varphi)$.

This new ground state manifests itself in several ways. Historically the disappearance of DC electrical resistivity was observed first [4]. As long as a material is superconducting, a current in the material will continue to flow. Secondly, superconductors behave subtly different in a magnetic field than one would expect for a material without electrical resistance. Instead of a perfect diamagnet an external field is shielded from the interior of a superconductor by surface currents. If a material is cooled through the critical temperature $T_{c}$ in a static background field, surface currents will flow spontaneously to cancel this field in the interior. This is distinct from Lenz's law, where currents will only flow in reaction to a dynamic field, and this effect is known as the Meissner-Ochsenfeld effect [5]. 
For the experiments presented in this thesis, perhaps the most used aspects of superconducting systems are flux quantisation and the Josephson effect due to the proximity effect.

Flux quantisation is the result of the macroscopic quantum state in a superconductor. The wave function describing the collective behaviour of the Cooper pairs is single-valued around any contour, which results in flux quantisation with $\Phi_{0}=\mathrm{h} / 2 \mathrm{e}$ [6]. This quantisation can be probed in several ways, such as by measuring the magnetisation $[7,8]$, the resistance or the transition temperature [9] of a superconducting ring. Flux quantisation is an example of a topological quantum number, insensitive to disturbances such as the geometry of the ring [10].

On the interface between a superconductor and non-superconducting material, the wave function does not diminish abruptly but extends for a distance characterised by the coherence length $\xi_{N}[11,12]$. When a non-superconducting material is wedged between two superconductors such that these wave functions interfere, Josephson predicted that the two could become phase-coherent [13-16]. The behaviour of this contact is now dependent on the phase difference between the two superconductors. The DC Josephson effect states that the sine of the phase difference between the two superconductors modulates the supercurrent, or maximum current without a voltage. The modulation is periodic in $2 \pi$ with the phase difference. The time derivative of the phase difference leads to a voltage difference, inversely proportional with $\Phi_{0}$. This is known as the AC Josephson effect. Despite possible defects and irregularities in a Josephson junction, this relationship between the time derivative of the phase and the voltage is exact, and for this reason used as the conventional definition of the volt, $\mathrm{V}_{90}$.

\subsection{TOPOLOGICAL INSULATORS}

Another system where topology plays a defining role in determining the physical behaviour is a topological insulator. Strong spin-momentum locking in these materials, which have a bulk insulating band structure, results in surface states which host helical Dirac fermions [17]. Inducing superconductivity in these states could result in a p-wave order parameter, and Majorana zero modes [18-20]. This latter effect is actively under investigation in a wide variety of systems such as nanowires [21, 22], HgTe systems [23-26] and the surfaces of bismuth based topological insulators (chapter 2). Whereas effects due to topol- 
ogy, such as flux quantisation in a superconductor and helical Dirac fermions in a topological insulator, are robust, combining these two materials does not easily lead to unambiguous results despite a clear prediction for a modified flux quantisation, $\mathrm{h} / \mathrm{e}$. These difficulties are attributed to quasi-particle poisoning, quantum phase slips, non-perpendicular bound states and contributions from non-Majorana modes in the junctions, and there is a continuous search for unambiguous experiments and signatures [27-32].

The connection between bismuth based topological insulators and superconductors is introduced in more detail in chapter 2 . In chapter 3 the interplay between geometric constraints, namely the width of planar Josephson junctions, and the search for signatures of topological superconductivity in $\mathrm{Nb} / \mathrm{Bi}_{2} \mathrm{Te}_{3}$ systems is explored. One of the constraints of the first generation Bi-based topological insulators is the presence of bulk conductivity due to defects. Chapter 4 presents the realisation of Josephson contacts on a topological insulator without a bulk shunt, $\mathrm{Bi}_{2-\chi} \mathrm{Sb}_{\chi} \mathrm{Te}_{3-y} \mathrm{Se}_{y}$. To investigate the phase dependence of $\mathrm{Nb} / \mathrm{Bi}_{2} \mathrm{Te}_{3}$ Josephson junctions, SQUID devices allow for control of the phase of the junctions. By comparing the geometric areas of the SQUID loop and the Josephson junctions the periodicity of the current-phase relationship of the junctions is determined in chapter 5 . In the final chapter concerning superconductor / topological insulator systems, chapter 6, SQUID characteristics of superconductors with different current-phase relationships are modelled.

\subsection{JOSEPHSON JUNCTION ARRAYS}

Another way to exploit the macroscopic wave function present in superconductors is by creating a superconducting lattice or array. Such a superconducting network is relevant both from a technical and fundamental point of view. In the definition of a voltage standard using the Josephson effect, large arrays of coupled Josephson junctions are used for frequency-to-voltage conversion, exploiting the precision in $\Phi_{0}$ mentioned previously. In fundamental physics, superconducting networks can be used as a model for disordered superconductors, investigating phase transitions and as model systems for engineered Hamiltonians [33-35].

The starting point for the experiments presented in chapters 7 and 8 is an array of superconducting islands connected via the 
proximity effect on a metallic substrate. The behaviour of these simple structures, described by the Little-Parks effect or SQUID physics, becomes rich and complex due to vortices, topologically stable excitations, threading the unit cell, or loops, of the arrays. The superconducting array creates an egg-crate potential landscape for vortices, with minima in between islands. In zero field, the interactions between thermally activated vortices and anti-vortices are associated with a Berezinskii-KosterlitzThouless (BKT) transition as a function of temperature. In an applied magnetic field, the vector field places competing requirements on the order parameter of islands which are part of different loops, and the resulting vortices will arrange themselves throughout the system. To minimise the total energy the distance between vortices will be maximised. In a thin superconducting film or strips [36] this is evident by the formation of an Abrikosov lattice [37]. The number of vortices in an array can be controlled by a perpendicular magnetic field. Similarly, when the number of vortices per unit cell is a rational fraction, stable and periodic vortex lattices exist which are insensitive to disturbances. Magnetic fields which lead to such periodic vortex configurations are commensurate with the physical lattice. In chapter 7 , the existence and behaviour of these periodic vortex lattices is investigated and found to behave as a Mott system. In the final chapter the influence of disturbing the lattice by removing islands is measured and analysed, probing the relationship between disorder, commensurability and superconductivity.

BIBLIOGRA PHY

[1] J. Bardeen, L. N. Cooper, and J. R. Schrieffer, Phys. Rev. 106, 162 (1957).

[2] J. Bardeen, L. N. Cooper, and J. R. Schrieffer, Phys. Rev. 108, 1175 (1957).

[3] L. N. Cooper, Phys. Rev. 104, 1189 (1956).

[4] H. Onnes, Commun. Phys. Lab. Univ. Leiden 12, 120 (1911).

[5] W. Meissner and R. Ochsenfeld, Naturwissenschaften 21, 787 (1933).

[6] A. de Waele and R. de Bruyn Ouboter, Physica 41, 225 (1969). 
[7] B. S. Deaver and W. M. Fairbank, Phys. Rev. Lett. 7, 43 (1961).

[8] R. Doll and M. Näbauer, Phys. Rev. Lett. 7, 51 (1961).

[9] W. A. Little and R. D. Parks, Phys. Rev. Lett. 9, 9 (1962).

[10] D. J. Thouless, Topological Quantum Numbers in Nonrelativistic Physics (World Scientific Publishing, 1997).

[11] P. G. de Gennes, Rev. Mod. Phys. 36, 225 (1964).

[12] P. G. de Gennes, Superconductivity of Metals and Alloys (Addison-Wesley Publishing Company, 1966).

[13] B. D. Josephson, Physics Letters 1, 251 (1962).

[14] P. W. Anderson and J. M. Rowell, Phys. Rev. Lett. 10, 230 (1963).

[15] B. D. Josephson, Rev. Mod. Phys. 46, 251 (1974).

[16] K. K. Likharev, Rev. Mod. Phys. 51, 101 (1979).

[17] D. Hsieh, Y. Xia, D. Qian, L. Wray, J. H. Dil, F. Meier, J. Osterwalder, L. Patthey, J. G. Checkelsky, N. P. Ong, A. V. Fedorov, et al., Nature 460, 1101 (2009).

[18] A. Y. Kitaev, Physics-Uspekhi 44, 131 (2001).

[19] L. Fu and C. L. Kane, Phys. Rev. Lett. 100, 096407 (2008).

[20] M. Z. Hasan and C. L. Kane, Rev. Mod. Phys. 82, 3045 (2010).

[21] V. Mourik, K. Zuo, S. M. Frolov, S. R. Plissard, E. P. A. M. Bakkers, and L. P. Kouwenhoven, Science 336, 1003 (2012).

[22] L. P. Rokhinson, X. Liu, and J. K. Furdyna, Nat Phys 8, 795 (2012).

[23] L. Maier, J. B. Oostinga, D. Knott, C. Brüne, P. Virtanen, G. Tkachov, E. M. Hankiewicz, C. Gould, H. Buhmann, and L. W. Molenkamp, Phys. Rev. Lett. 109, 186806 (2012).

[24] J. Reuther, J. Alicea, and A. Yacoby, Phys. Rev. X 3, 031011 (2013). 
[25] S. Hart, H. Ren, T. Wagner, P. Leubner, M. Mühlbauer, C. Brüne, H. Buhmann, L. W. Molenkamp, and A. Yacoby, ArXiv e-prints p. 1312.2559 (2013).

[26] J. B. Oostinga, L. Maier, P. Schüffelgen, D. Knott, C. Ames, C. Brüne, G. Tkachov, H. Buhmann, and L. W. Molenkamp, Phys. Rev. X 3, 021007 (2013).

[27] D. M. Badiane, M. Houzet, and J. S. Meyer, Phys. Rev. Lett. 107, 177002 (2011).

[28] R. W. Reinthaler, P. Recher, and E. M. Hankiewicz, Phys. Rev. Lett. 110, 226802 (2013).

[29] C. Beenakker, Annual Review of Condensed Matter Physics 4, 113 (2013).

[30] G. Tkachov and E. M. Hankiewicz, physica status solidi (b) 250 (2013).

[31] G. Tkachov and E. M. Hankiewicz, Phys. Rev. B 88, 075401 (2013).

[32] S.-P. Lee, K. Michaeli, J. Alicea, and A. Yacoby, ArXiv eprints p. 1403.2747 (2014).

[33] T. I. Baturina, V. M. Vinokur, A. Y. Mironov, N. M. Chtchelkatchev, D. A. Nasimov, and A. V. Latyshev, EPL (Europhysics Letters) 93, 47002 (2011).

[34] R. Newrock, C. Lobb, U. Geigenmüller, and M. Octavio, Solid State Physics 54, 263 (2000).

[35] P. Martinoli and C. Leemann, Journal of Low Temperature Physics 118, 699 (2000).

[36] K. H. Kuit, J. R. Kirtley, W. van der Veur, C. G. Molenaar, F. J. G. Roesthuis, A. G. P. Troeman, J. R. Clem, H. Hilgenkamp, H. Rogalla, and J. Flokstra, Phys. Rev. B 77, 134504 (2008).

[37] A. Abrikosov, Journal of Physics and Chemistry of Solids 2, 199 (1957). 
INTRODUCTION TO BISMUTH BASED

TOPOLOGICAL INSULATOR -

SUPERCONDUCTOR SYSTEMS

The surface of a $3 \mathrm{D}$ topological insulator is conducting and the topologically non-trivial nature of the surface states is observed in experiments. It is the aim of this introduction to review and analyse experimental observations of the superconducting transport properties of hybrid structures consisting of superconductors and these topological insulators. Superconductivity can be induced in topological superconductors by means of the proximity effect. The induced supercurrents, Josephson effects and currentphase relations will be reviewed.

\subsection{INTRODUCTION}

A three-dimensional (3D) topological insulator (TI) is a semiconductor with two-dimensional (2D) surface states that have an energy dispersion across the entire bulk bandgap. These surface states arise from band inversion, due to strong spinorbit interactions. The band inversion makes the surface states topologically nontrivial, meaning that these states are protected (cannot be removed) and that the states have a helical Diractype dispersion, where spin is tightly coupled to momentum. Several compounds have been theoretically predicted to be $3 \mathrm{D}$ TIs [1]. Strong experimental evidence for the presence of topological surface states exists for the class of Bi-based materials: the alloy $\mathrm{Bi}_{1-\chi} \mathrm{Sb}_{x}$ [2], and the compounds $\mathrm{Bi}_{2} \mathrm{Se}_{3}$ and $\mathrm{Bi}_{2} \mathrm{Te}_{3}$ [3], see Fig. 2.I for the crystal structure. The class of Bi-based topological insulator has been expanded with the fabrication

This chapter is published as part of "Magnetotransport and induced superconductivity in Bi based three-dimensional topological insulators" by M. Veldhorst, M. Snelder, M. Hoek, C. G. Molenaar, D. P. Leusink, A. A. Golubov, H. Hilgenkamp \& A. Brinkman, Phys. Status Solidi RRL 7:26-38 (2013). The author of this PhD thesis has contributed to this publication by contributing in the writing of the sections presented in this chapter. 


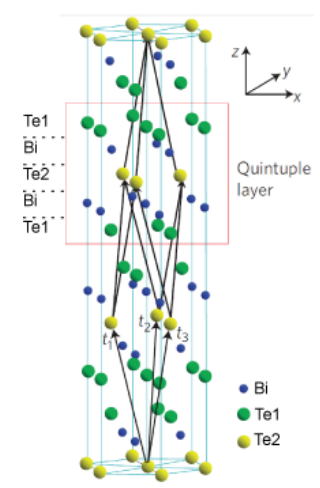

Figure 2.1: The CRYSTAL STRUCtURE OF $\mathrm{BI}_{2} \mathrm{TE}_{3}$ AS A REPRESENTATIVE EXAMPLE OF Bi-based 3 D topological insulators. From [3].

of $\mathrm{TlBiSe}_{2}$ [4-6], $\mathrm{TlBiTe}_{2}$ [6], BiSbPbTe [7], and $\mathrm{PbBiTe}_{4}$ [8]. The bulk of these compounds is not necessarily insulating, due to the presence of defects and impurities. This bulk conductivity might shunt and mask topological transport properties. A successful trend in the research on topological insulators has been to reduce the number of defects and impurities by atom substitutions such as $\mathrm{Bi}_{2} \mathrm{Te}_{2} \mathrm{Se}$ [9-11] and $\mathrm{Bi}_{2-\chi} \mathrm{Sb}_{\chi} \mathrm{Te}_{3-y} \mathrm{Se}_{y}$ [12] which have higher bulk resistivities. Simultaneously, progress has been made in disentangling topological surface state transport properties from bulk transport contributions.

The topologically non-trivial surface state is an attractive medium to study unconventional superconductivity. When superconductivity exists in a system with a Dirac-type dispersion and helical spin-momentum locking, exotic features could be realised such as $p$-wave order parameter symmetry [13] and Majorana type bound states [14]. Josephson hybrid structures have been designed theoretically already to mimic non-abelian particle statistics [14]. Parallel to the trend of doping and alloying TIs to render them superconducting $[15,16]$ runs the successful approach of inducing superconductivity from a standard superconductor into a TI by means of the proximity effect. Here, we will review the current status of the experimental efforts in the latter direction. Evidence for supercurrents through topological surface states will be analysed, also in the presence of bulk conducting channels. The prospects for future Superconducting quantum interference device (SQUID) based experiments to detect topological effects in the superconductivity will be discussed. 


\subsection{TOPOLOGICAL INSULATORS WITH PROXIMITY INDUCED SUPERCONDUCTIVITY}

The combination of superconductivity with spin-orbit coupling opens many new exciting research directions, including the realisation of a new emergent particle: the Majorana bound state. This particle, which is its own antiparticle, emerges from the electron-hole symmetry of superconducting condensation and the spin-momentum locking in the topological insulator [14, 17]. The high potential of this particle in quantum computation led to many new proposals in various material systems, such as topological insulator/superconductor structures [14, 18-22], spin-triplet p-wave superconductors $[13,23-26]$, helical superconductors $[27,28]$, topological superconductors [15, 16, 29-31], semiconductor/metals with strong orbit coupling in combination with superconductors or superconductors with strong spin orbit coupling [32-39]. In the latter category the most wellknown device is inducing superconductivity in nanowires structures with strong Zeeman and Rashba fields [37-56]. First signatures of the Majorana fermion are revealed [40-42, 57-62] but in order to test all its peculiar properties the realisation of superconducting interference devices will be a prerequisite $[52,63]$.

\subsubsection{Supercurrents through TI structures}

Shortly after the discovery of the Bi-compound topological insulators, observations of supercurrents in superconductor - topological insulator structures have been reported [64-70], as well as the coexistence of superconductivity and topological surface states [71]. Several fabrication techniques have been used such as epitaxial film growth and mechanical exfoliation for the topological insulator structure. Superconducting electrodes are deposited on top resulting in lateral junctions. Sandwich-type junctions have been realised by Qu et al. [66] using a clever fabrication method based on exfoliated flakes. Using an oxidised Si substrate, Sacépé et al. [64] demonstrated gate tunable supercurrents. The change in the $I_{c} R_{N}$ product is of the order of $15 \%$ by applying a gate voltage between $-80 \mathrm{~V}$ and $+50 \mathrm{~V}$. Structures based on exfoliation are therefore flexible and have high potential. High quality topological insulators with small thicknesses and smooth surfaces can be realised that allow the construction of multiple devices on a single flake [65]. Orlyanchik et al. [72] demonstrated a gate tunable supercurrent with an abrupt 
change at the band-inversion point. Kurter et al. [73] find that at this band-inversion point, the modulation depth of dc SQUIDs also changes, without a change in the periodicity.

The characteristic $I_{C} R_{N}$ product observed so far is well below the characteristic voltage scale of $\pi \Delta / e$, e.g. $5 \mathrm{mV}$ for superconducting niobium electrodes. Although mechanisms as scattering and electron-hole decoherence due to junction lengths longer than $\xi$ can lower the $I_{c} R_{N}$ product, these junctions often have high interface transparencies and junction lengths comparable to $\xi$. In these systems, an important mechanism lowering the $I_{c} R_{N}$ product is the presence of bulk conduction. Normal state transport is dominated by the bulk states which are spread over the entire sample. As the electrodes are fabricated on top, proximity induced superconductivity will be the strongest on the top, and the bulk is effectively shunting the junction $R_{N}$ significantly, thereby reducing the $I_{c} R_{N}$ product. Veldhorst et al. [65] obtained a bulk mean free path shorter than the junction length while the system is in the ballistic limit and concluded that the discrimination between bulk and surface states can be so strong that the top topological surface state dominates the superconducting transport, while the bulk states dominate the normal state.

It is an intriguing question why this discrimination is so strong. Besides geometrical effects, strong anisotropy of the band structure of Bi-based topological insulators is a possible origin. The ratio of the superconducting coherence length $\xi_{z} / \xi_{x y}$ in the topological insulator depends on the effective mass and the potential energy difference along the $x y$-direction and $z$ direction. From the expression of the coherence-length tensor for anisotropic superconductors as described in Ref. [74], we find for the proximity coherence length in the non-superconducting material

$$
\frac{\xi_{z}}{\xi_{x y}} \propto \frac{m_{x y}}{m_{z}} \frac{k_{z}}{k_{x y}} \propto \frac{m_{x y}}{m_{z}} \frac{a}{c}
$$

where the $x, y$ and $z$ axis are as defined in Fig. 2.1. The superconductivity is induced in the surface states in the $x y$-plane. $a$ and $c$ are the lattice constants of the unit cell along the $x$ (or $y$ ) and $z$ direction, respectively. Using the valence band effective masses $m_{x y}=0.18$ and $m_{z}=0.84$ for $\mathrm{Bi}_{2} \mathrm{Te}_{3}[75]$ in conjunction with the lattice constant differences, we find a ratio $\xi_{z} / \xi_{x y}$ of 0.15 . Hence, the band structure itself causes a strong anisotropic proximity effect. An additional argument for strong 
anisotropy is the observation of the layered quantum Hall effect in $\mathrm{Bi}_{2} \mathrm{Te}_{3}$ [76]. Furthermore, the effective parameter that determines the suppression of $I_{c} R_{N}$ in S-N-S junctions (whether ballistic or diffusive, and including barriers at interfaces) is given by $\gamma_{\text {eff }}=\left(\frac{L}{\xi}\right)^{2} \frac{R_{B}}{R_{N}}$, with the boundary resistance $R_{B}$ and the interlayer resistance $R_{N}$ [77]. From this suppression parameter it can already be seen that a low interlayer resistance with respect to the boundary resistance gives a high $\gamma_{\text {eff }}$. The $I_{c} R_{N}$ is then suppressed more strongly for the bulk than for the surface channel. Finally, topological effects might render the interfaces to the surface states intrinsically transparent. These intriguing options open a new exciting research direction and give the opportunity to have surface states dominating the supercurrent in topological insulators even in the presence of substantial bulk conductivity.

Successive Andreev reflections allow quasiparticles to escape from the superconducting gap in the voltage state. These multiple Andreev reflection (MAR) processes cause structures in the current voltage characteristics at voltages $\mathrm{eV}=\alpha \Delta / \mathrm{n}$, with $\mathrm{n}$ an integer and $\alpha=2$ for standard Cooper pair tunneling and $\alpha=1$ for single electron tunneling mediated by Majorana fermions. Experimentally, Zhang et al. [67] have reported evidences for MAR for junctions that are in the standard $\alpha=2$ regime (as expected for $3 \mathrm{D}$ junctions). Remarkably, in this experiment only steps are observed for even $n$.

\subsubsection{Current-phase relationship}

Topological protection causes interfaces to be highly transparent due to Klein tunneling [14, 17] and absence of backscattering for perpendicular incidence. This protection can change the standard current-phase relationship in Josephson junctions from $\sin (\phi / \alpha)$ with $\alpha=1$ to $\alpha=2$. The systems is then gapless at zero energy, and there exists a Majorana bound state when the phase difference $\phi$ across the junction equals $\pi$. Many theoretical proposals have been put forward to reveal this currentphase relationship. These proposals are based on the AC and DC Josephson effects and superconducting quantum interference devices with topological interlayers.

The AC Josephson effect has been observed by Veldhorst et al. [65], see Fig. 2.2. These Josephson junctions show clear steps when irradiated with microwaves, demonstrating the Josephson nature of the supercurrent and from the spacing between 


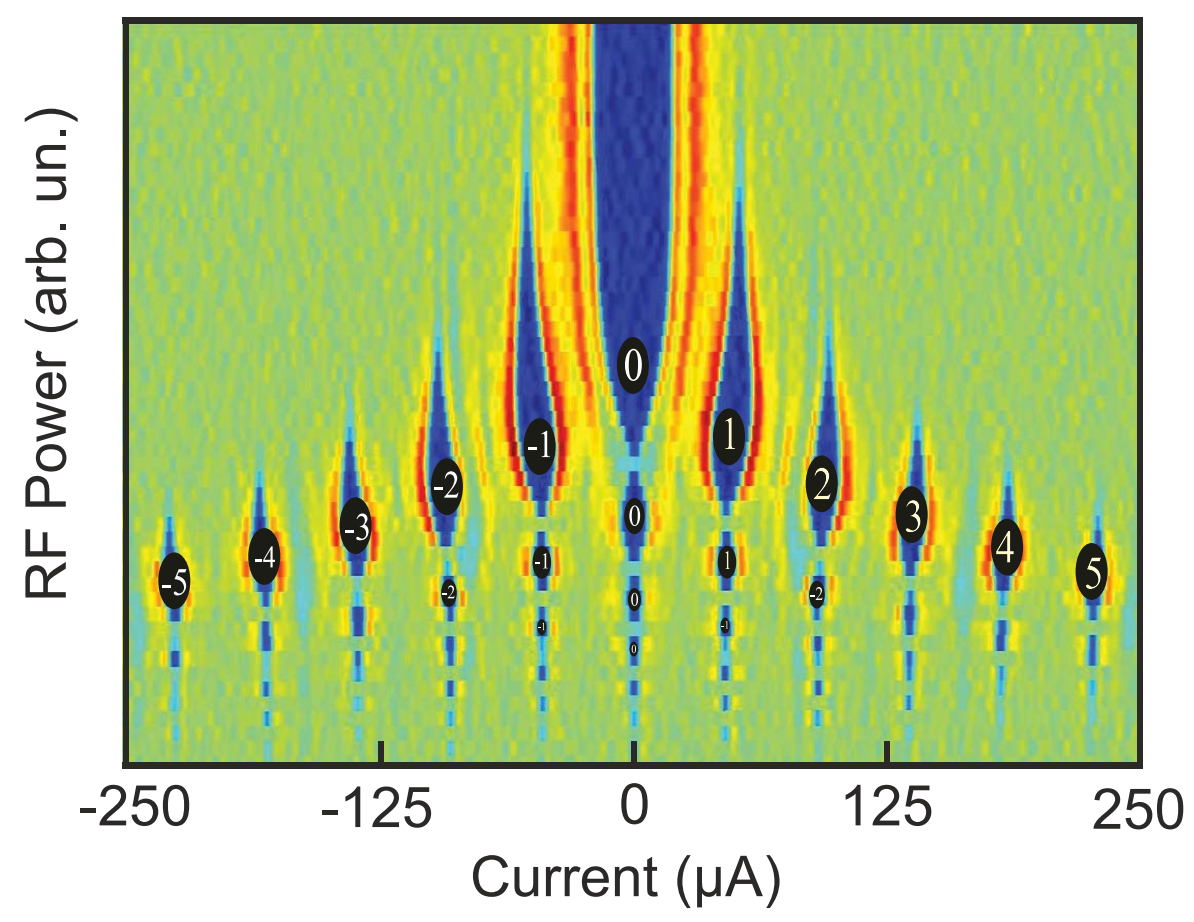

Figure 2.2: BESSEL-PEACOCK COLOR PLOT OF THE CONDUCTANCE AFTER IRRADIATION WITH MiCROWAVES (10.o GHz). From [65].

the steps it can be concluded that these measurements are in the $\alpha=1$ regime. Future strategies to enhance the fraction of $\sin (\phi / 2)$ tunneling involve non-equilibrium measurements [78] and the suppression of non-perpendicular trajectories in the junctions [79].

The DC Josephson effect causes a modulation of the superconducting critical current in an applied magnetic field. In the limit of infinite width and a homogenous current density distribution the magnetic field dependence of the critical current is the Fraunhofer sinc function:

$$
\mathrm{I}_{\mathrm{c}}\left(\Phi_{0}\right)=\mathrm{I}_{\mathrm{c}}(0) \frac{\sin \left(\pi \Phi / \alpha \Phi_{0}\right)}{\pi \Phi / \alpha \Phi_{0}}
$$

Here, $\Phi_{0}$ is the superconducting flux quantum. Fig. 2.3 shows typical critical current modulation patterns observed so far. The Josephson junctions fabricated by Qu et al. [66] are well described by Eq. (2.2) with $\alpha=1$, while Veldhorst et al. [65] and Williams et al. [69] report deviations.

Deviations in the field dependence can result from flux focusing effects and geometrical inhomogeneities, e.g. pinholes result in a slower decay of the side lobes, while lower current densities at the edges result in faster decay of the side lobes. Furthermore, having a finite width changes the field depen- 


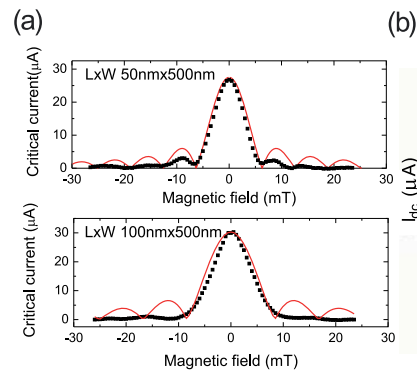

(b)

(c)

Figure 2.3: CRITICAL CURRENT MODUlation BY MAGNETIC FIELD OF JOSEPHSON JUNCTION WITH TOPOLOGICAL INSULATOR INTERlayers. (a) From [65]. (b) From [66]. (c) From [69]. While the Josephson junctions fabricated by Qu et al. [66] (b) have standard Fraunhofer patterns, the junctions from Veldhorst et al. [65] (a) and Williams et al. [69] (c) show deviations. Deviations occur both with respect to the field period (which is smaller than expected from the effective junction area) and in the shape.

dence, which becomes significant when $W \approx L$, the situation for most experiments so far. The ratio between the length $L$ and the width $W$ of the junctions can change both the magnitude and period of the diffraction pattern [8o]. The period increases from $\Phi_{0}$ to $2 \Phi_{0}$ for $\mathrm{L} / \mathrm{W} \rightarrow \infty$ [81] when the junction edges are 'open', as in Fig. 2.4(a). In this scenario, the side lobes will decrease more rapidly. This scenario is applied in chapter 4 .

When specular reflection occurs at the edge of the junction, such as in Fig. 2.4(b), the $2 \Phi_{0}$ crossover occurs at smaller aspect ratios [82]: $L / W \sim 1$. The ratio that is important in this scenario is the distance between the Josephson vortex and the range of nonlocal electrodynamics, determined by the thermal length $\xi_{N}$. As long as the range of nonlocal electrodynamics is smaller than the distance between vortices, the $\Phi_{0}$ period remains. For a larger range or strong nonlocality the period becomes $2 \Phi_{0}$ due to boundary effects. Including a finite tunneling barrier to this geometry mainly causes a sharpening of the Fraunhofer pattern and the side lobs are flattened [83]. This flattening is also characteristic for SNS junctions in the diffusive limit [84].

A third situation occurs when the magnetic field is parallel to the surface but perpendicular to the current as shown in Fig. 2.4(c). This changes the Fraunhofer pattern drastically [85], including irregular periods, periods smaller than the flux quantum and non-zero minima depending on the L/D ratio. The experimentally realised dc SQUIDs, described in chapter 5 , based 
(a)

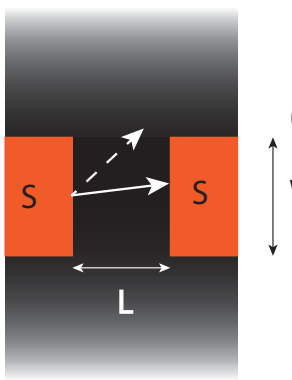

(b)

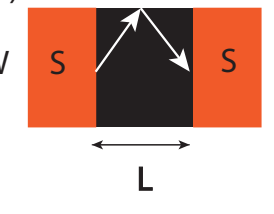

(c)

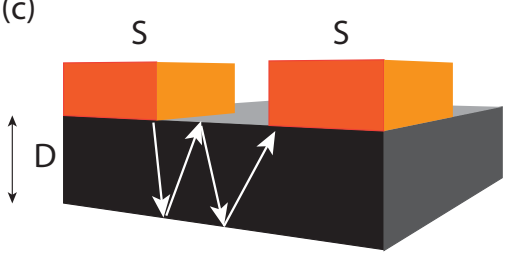

Figure 2.4: GeOMETRIC EFFECTs. (a) Geometry of S-TI-S junctions as used in the calculation of Ref. [81] to determine the critical current modulation by applied magnetic fields. The dotted arrow shows the path of an electron that never goes to the other superconductor but leaves the junction. In the middle of the superconductor the angle for which the electron still reach the other superconductor is larger than at the edges. (b) Geometry as used in the calculation of Ref [82]. At the boundaries the electrons are specular reflected. (c) The geometry as used in the calculation of Ref. [85].

on junctions where the critical current field dependency deviates from the standard Fraunhofer pattern [65] show standard fluxoid quantisation and suggest that the junction deviations are due to geometrical effects.

The observation of Fraunhofer patterns in topological Josephson junctions unambiguously shows the development of the superconducting proximity effect in the topological insulator. The deviations from the standard Fraunhofer form an interesting platform to search for new phenomena in these junctions. However, these junctions are nanosized, are often ballistic, and multiple bands can contribute to the conduction, resulting in complex scenarios. Still, based on these Josephson junctions SQUIDs can be fabricated that can discriminate between inhomogeneities and transport mediated by Majorana fermions.

\subsection{3 dc SQUIDs with unconventional current phase relationships}

Due to the appearance of Majorana fermions single electron tunneling occurs and the resulting current-phase relation of Josephson junctions can become $4 \pi$-periodic. The $4 \pi$-periodic current-phase relationship of topological Josephson junctions has its signatures in superconducting loops [86]. In the absence of relaxation mechanisms, standard fluxoid quantisation is doubled. Unfortunately, mechanisms such as quasiparticle poisoning and quantum phase slips will drive the system easily to 
(a)
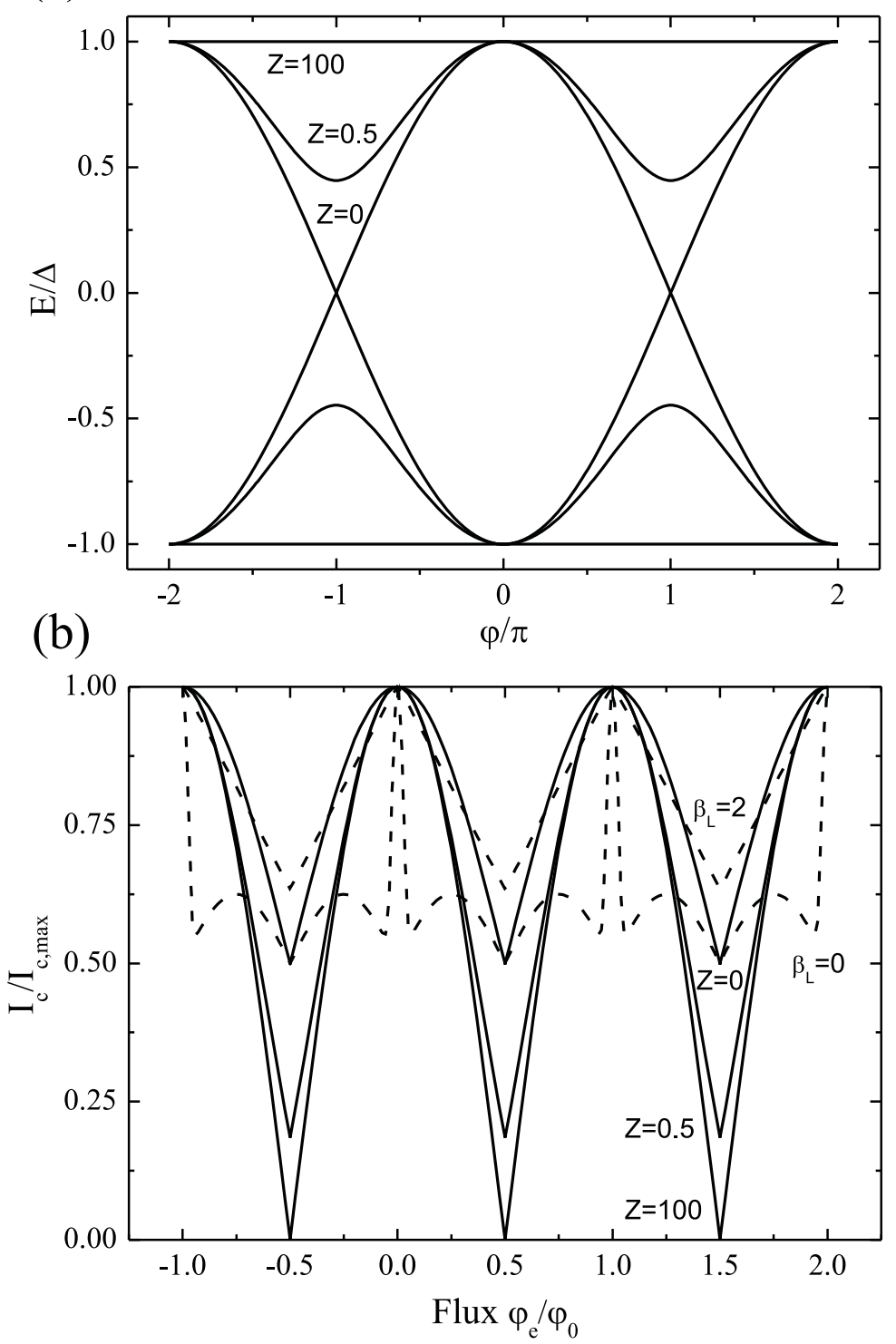

Figure 2.5: SQUIDS INCLUDING TRANSPARANT JUNCTIONS. (a) Currentphase relationship of junctions with different interface transparancies ( $Z=0,0.5$ and 100). (b) Resulting SQUID characteristics. Dashed lines include the peaked current phase relationship as considered in the phenomenological model introduced by Williams et al. [69], with the screening parameter $\beta_{\mathrm{L}}=0$ and 2 . These results hold in the presence of relaxation mechanisms and are independent of junction homogeneities and are thereby strong signatures of unconventional current-phase relationships. 
standard fluxoid quantisation. However, as shown in chapter 6 , even in this regime the unusual current phase relation of the individual junctions alter the standard SQUID modulation characteristics. This model is based on imposing fluxoid quantisation on a superconducting loop interrupted by two Josephson junctions described with the resistivily and capacitively shunted junction (RCSJ) model. To further illustrate this model we show the scenario of full relaxation in Fig. 2.5, where the junctions have been modeled by the Blonder-Tinkham-Klapwijk (BTK) approach [87]. The resulting bound states are described by $E= \pm \sqrt{\frac{\cos (\phi / 2)^{2}+Z^{2}}{1+Z^{2}}}$. Here, $Z$ is the BTK barrier strength of the barriers I. These results also represent bound states of superconductor - topological insulator Josephson junctions, where the factor $Z$ effectively describes the scattering resulting from finite angle incidence with momentum mismatches and the appearance of a magnetic gap. In the standard SQUID scenario, the Josephson junctions have low transparency (corresponding to $Z=100$ in Fig. 2.5) and can be described with a $\sin (\phi)$ current-phase relationship. In that case the dc SQUID has a sinusoidal critical current modulation as a function of magnetic field and standard fluxoid quantisation $\Phi_{0}=\mathrm{h} / 2 e$. Lowering the barrier strength, increasing the interface transparency, results in an incomplete critical current modulation [88]. This incomplete modulation is a strong signature, since it is independent of the junction homogeneity and survives up to the regime of energy relaxation (where doubled fluxoid quantisation is absent [17]).

We also included the current-phase relationship as imposed phenomenologically by Williams et al. [69]. This model assumes a current-phase relation that peaks when the relative phase over the junction equals $\pi$. In the regime of small self-induced flux, described by the screening parameter $\beta_{\mathrm{L}}=2 \pi \mathrm{LI}_{\mathrm{c}} / \Phi_{0}$, the characteristic spikes are also present in the dc SQUID characteristics. Increasing $\beta_{\mathrm{L}}$ smears out the spikes, resulting in a triangular critical current modulation, see Fig. 2.5. We conclude this section by noting that dc SQUIDs are ideal candidates to measure the unusual current-phase relationships of superconductor-topological insulator structures even in the presence of junction inhomogeneities and relaxation mechanisms. These dc SQUIDs can be used to test whether the critical current field dependences of topological junctions are due to intrinsic or extrinsic effects. 
[1] L. Fu, C. L. Kane, and E. J. Mele, Phys. Rev. Lett. 98, 106803 (2007).

[2] L. Fu and C. L. Kane, Phys. Rev. B 76, 045302 (2007).

[3] H. Zhang, C.-X. Liu, X.-L. Qi, X. Dai, Z. Fang, and S.-C. Zhang, Nat Phys 5, 438 (2009).

[4] T. Sato, K. Segawa, H. Guo, K. Sugawara, S. Souma, T. Takahashi, and Y. Ando, Phys. Rev. Lett. 105, 136802 (2010).

[5] K. Kuroda, M. Ye, A. Kimura, S. V. Eremeev, E. E. Krasovskii, E. V. Chulkov, Y. Ueda, K. Miyamoto, T. Okuda, K. Shimada, H. Namatame, et al., Phys. Rev. Lett. 105, 146801 (2010).

[6] Y. L. Chen, Z. K. Liu, J. G. Analytis, J.-H. Chu, H. J. Zhang, B. H. Yan, S.-K. Mo, R. G. Moore, D. H. Lu, I. R. Fisher, S. C. Zhang, et al., Phys. Rev. Lett. 105, 266401 (2010).

[7] S. Souma, K. Eto, M. Nomura, K. Nakayama, T. Sato, T. Takahashi, K. Segawa, and Y. Ando, Phys. Rev. Lett. 108, 116801 (2012).

[8] K. Kuroda, H. Miyahara, M. Ye, S. V. Eremeev, Y. M. Koroteev, E. E. Krasovskii, E. V. Chulkov, S. Hiramoto, C. Moriyoshi, Y. Kuroiwa, K. Miyamoto, et al., Phys. Rev. Lett. 108, 206803 (2012).

[9] S.-Y. Xu, L. A. Wray, Y. Xia, R. Shankar, A. Petersen, A. Fedorov, H. Lin, A. Bansil, Y. S. Hor, D. Grauer, R. J. Cava, et al., ArXiv e-prints 1007.5111 (2010).

[10] Z. Ren, A. A. Taskin, S. Sasaki, K. Segawa, and Y. Ando, Phys. Rev. B 82, 241306 (2010).

[11] J. Xiong, A. Petersen, D. Qu, Y. Hor, R. Cava, and N. Ong, Physica E: Low-dimensional Systems and Nanostructures 44, 917 (2012).

[12] Z. Ren, A. A. Taskin, S. Sasaki, K. Segawa, and Y. Ando, Phys. Rev. B 84, 165311 (2011).

[13] A. Y. Kitaev, Physics-Uspekhi 44, 131 (2001). 
[14] L. Fu and C. L. Kane, Phys. Rev. Lett. 100, 096407 (2008).

[15] S. Sasaki, M. Kriener, K. Segawa, K. Yada, Y. Tanaka, M. Sato, and Y. Ando, Phys. Rev. Lett. 107, 217001 (2011).

[16] E. van Heumen, S. Johnston, J. Kaas, N. de Jong, F. Massee, J. Oen, E. Rienks, A. Varykhalov, J. B. Goedkoop, Y. Huang, and M. S. Golden, ArXiv e-prints 1110.4406 (2011).

[17] L. Fu and C. L. Kane, Phys. Rev. B 79, 161408 (2009).

[18] L. Fu and C. L. Kane, Phys. Rev. Lett. 102, 216403 (2009).

[19] A. R. Akhmerov, J. Nilsson, and C. W. J. Beenakker, Phys. Rev. Lett. 102, 216404 (2009).

[20] Y. Tanaka, T. Yokoyama, and N. Nagaosa, Phys. Rev. Lett. 103, 107002 (2009).

[21] J. Linder, Y. Tanaka, T. Yokoyama, A. Sudbø, and N. Nagaosa, Phys. Rev. Lett. 104, 067001 (2010).

[22] K. T. Law, P. A. Lee, and T. K. Ng, Phys. Rev. Lett. 103, 237001 (2009).

[23] N. Read and D. Green, Phys. Rev. B 61, 10267 (2000).

[24] C. J. Bolech and E. Demler, Phys. Rev. Lett. 98, 237002 (2007).

[25] K. Sengupta, I. Žutić, H.-J. Kwon, V. M. Yakovenko, and S. Das Sarma, Phys. Rev. B 63, 144531 (2001).

[26] Y. E. Kraus, A. Auerbach, H. A. Fertig, and S. H. Simon, Phys. Rev. B 79, 134515 (2009).

[27] M. Sato and S. Fujimoto, Phys. Rev. B 79, 094504 (2009).

[28] Y. Tanaka, T. Yokoyama, A. V. Balatsky, and N. Nagaosa, Phys. Rev. B 79, 060505 (2009).

[29] L. Fu and E. Berg, Phys. Rev. Lett. 105, 097001 (2010).

[30] T. H. Hsieh and L. Fu, Phys. Rev. Lett. 108, 107005 (2012).

[31] A. Yamakage, K. Yada, M. Sato, and Y. Tanaka, Phys. Rev. B 85, 180509 (2012).

[32] M. Sato, Y. Takahashi, and S. Fujimoto, Phys. Rev. Lett. 103, O20401 (2009). 
[33] M. Sato, Y. Takahashi, and S. Fujimoto, Phys. Rev. B 82, 134521 (2010).

[34] A. C. Potter and P. A. Lee, Phys. Rev. Lett. 105, 227003 (2010).

[35] J. Linder and A. Sudbø, Phys. Rev. B 82, o85314 (2010).

[36] A. Yamakage, Y. Tanaka, and N. Nagaosa, Phys. Rev. Lett. 108, 087003 (2012).

[37] J. D. Sau, R. M. Lutchyn, S. Tewari, and S. Das Sarma, Phys. Rev. Lett. 104, 040502 (2010).

[38] T. D. Stanescu, J. D. Sau, R. M. Lutchyn, and S. Das Sarma, Phys. Rev. B 81, 241310 (2010).

[39] J. Alicea, Phys. Rev. B 81, 125318 (2010).

[40] R. M. Lutchyn, J. D. Sau, and S. Das Sarma, Phys. Rev. Lett. 105, 077001 (2010).

[41] J. D. Sau, S. Tewari, R. M. Lutchyn, T. D. Stanescu, and S. Das Sarma, Phys. Rev. B 82, 214509 (2010).

[42] Y. Oreg, G. Refael, and F. von Oppen, Phys. Rev. Lett. 105, 177002 (2010).

[43] J. Klinovaja and D. Loss, Phys. Rev. B 86, o85408 (2012).

[44] S. Tewari, T. D. Stanescu, J. D. Sau, and S. D. Sarma, New Journal of Physics 13, 065004 (2011).

[45] J. D. Sau, S. Tewari, and S. Das Sarma, Phys. Rev. B 85, 064512 (2012).

[46] T. D. Stanescu, R. M. Lutchyn, and S. Das Sarma, Phys. Rev. B 84, 144522 (2011).

[47] R. M. Lutchyn, T. D. Stanescu, and S. Das Sarma, Phys. Rev. Lett. 106, 127001 (2011).

[48] R. M. Lutchyn and M. P. A. Fisher, Phys. Rev. B 84, 214528 (2011).

[49] A. Golub, I. Kuzmenko, and Y. Avishai, Phys. Rev. Lett. 107, 176802 (2011).

[50] K. Flensberg, Phys. Rev. B 82, 180516 (2010). 
[51] A. Romito, J. Alicea, G. Refael, and F. von Oppen, Phys. Rev. B 85, 020502 (2012).

[52] J. Alicea, Reports on Progress in Physics 75, 076501 (2012).

[53] D. Sticlet, C. Bena, and P. Simon, Phys. Rev. Lett. 108, 096802 (2012).

[54] A. C. Potter and P. A. Lee, Phys. Rev. B 83, 184520 (2011).

[55] B. Zhou and S.-Q. Shen, Phys. Rev. B 84, 054532 (2011).

[56] K. T. Law and P. A. Lee, Phys. Rev. B 84, o81304 (2011).

[57] V. Mourik, K. Zuo, S. M. Frolov, S. R. Plissard, E. P. A. M. Bakkers, and L. P. Kouwenhoven, Science 336, 1003 (2012).

[58] E. Reich, Nature 483, 132 (2012).

[59] F. Wilczek, Nature 486, 195 (2012).

[6o] A. Das, Y. Ronen, Y. Most, Y. Oreg, M. Heiblum, and H. Shtrikman, Nat Phys 8, 887 (2012).

[61] L. P. Rokhinson, X. Liu, and J. K. Furdyna, Nat Phys 8, 795 (2012).

[62] M. T. Deng, C. L. Yu, G. Y. Huang, M. Larsson, P. Caroff, and H. Q. Xu, Nano Letters 12, 6414 (2012).

[63] C. Beenakker, Annual Review of Condensed Matter Physics 4, 113 (2013).

[64] B. Sacépé, J. B. Oostinga, J. Li, A. Ubaldini, N. J. Couto, E. Giannini, and A. F. Morpurgo, Nat Communications 2, 575 (2011).

[65] M. Veldhorst, M. Snelder, M. Hoek, T. Gang, V. K. Guduru, X. L. Wang, U. Zeitler, W. G. van der Wiel, A. A. Golubov, H. Hilgenkamp, and A. Brinkman, Nature Materials 11, 417 (2012).

[66] F. Qu, F. Yang, J. Shen, Y. Ding, J. Chen, Z. Ji, G. Liu, J. Fan, X. Jing, C. Yang, and L. Lu, Sci. Rep. 2 (2012).

[67] D. Zhang, J. Wang, A. M. DaSilva, J. S. Lee, H. R. Gutierrez, M. H. W. Chan, J. Jain, and N. Samarth, Phys. Rev. B 84, 165120 (2011). 
[68] M. Veldhorst, C. G. Molenaar, X. L. Wang, H. Hilgenkamp, and A. Brinkman, Applied Physics Letters 100, 072602 (2012).

[69] J. R. Williams, A. J. Bestwick, P. Gallagher, S. S. Hong, Y. Cui, A. S. Bleich, J. G. Analytis, I. R. Fisher, and D. Goldhaber-Gordon, Phys. Rev. Lett. 109, 056803 (2012).

[70] P. Zareapour, A. Hayat, S. Y. F. Zhao, M. Kreshchuk, A. Jain, D. C. Kwok, N. Lee, S.-W. Cheong, Z. Xu, A. Yang, G. Gu, et al., Nat Commun 3, 1056 (2012).

[71] M.-X. Wang, C. Liu, J.-P. Xu, F. Yang, L. Miao, M.-Y. Yao, C. L. Gao, C. Shen, X. Ma, X. Chen, Z.-A. Xu, et al., Science 336,52 (2012).

[72] V. Orlyanchik, M. P. Stehno, C. D. Nugroho, P. Ghaemi, M. Brahlek, N. Koirala, S. Oh, and D. J. Van Harlingen, ArXiv e-prints 1309.0163 (2013).

[73] C. Kurter, A. D. K. Finck, P. Ghaemi, Y. S. Hor, and D. J. Van Harlingen, Phys. Rev. B 9o, 014501 (2014).

[74] H. Meuth, Phys. Rev. B 38, 11923 (1988).

[75] B. Y. Yavorsky, N. F. Hinsche, I. Mertig, and P. Zahn, Phys. Rev. B 84, 165208 (2011).

[76] H. Cao, J. Tian, I. Miotkowski, T. Shen, J. Hu, S. Qiao, and Y. P. Chen, Phys. Rev. Lett. 108, 216803 (2012).

[77] A. A. Golubov, M. Y. Kupriyanov, and E. Il'ichev, Rev. Mod. Phys. 76, 411 (2004).

[78] D. M. Badiane, M. Houzet, and J. S. Meyer, Phys. Rev. Lett. 107, 177002 (2011).

[79] M. Snelder, M. Veldhorst, A. A. Golubov, and A. Brinkman, Phys. Rev. B 87, 104507 (2013).

[8o] J. P. Heida, B. J. van Wees, J. J. Kuipers, T. M. Klapwijk, and G. Borghs, Phys. Rev. B 57, 11911 (1998).

[81] V. Barzykin and A. M. Zagoskin, Superlattices and Microstructures 25, 797 (1999).

[82] U. Ledermann, A. L. Fauchère, and G. Blatter, Phys. Rev. B 59, R9027 (1999). 
[83] D. E. Sheehy and A. M. Zagoskin, Phys. Rev. B 68, 144514 (2003).

[84] F. Bergeret and J. Cuevas, Journal of Low Temperature Physics 153, 304 (2008).

[85] G. Mohammadkhani, M. Zareyan, and Y. M. Blanter, Phys. Rev. B 77, 014520 (2008).

[86] B. van Heck, F. Hassler, A. R. Akhmerov, and C. W. J. Beenakker, Phys. Rev. B 84, 180502 (2011).

[87] G. E. Blonder, M. Tinkham, and T. M. Klapwijk, Phys. Rev. B 25, 4515 (1982).

[88] M. Veldhorst, C. G. Molenaar, C. J. M. Verwijs, H. Hilgenkamp, and A. Brinkman, Phys. Rev. B 86, 024509 (2012). 
GEOMETRIC DEPENDENCE OF NB-BI $\mathrm{TE}_{3}-\mathrm{NB}$

TOPOLOGICAL JOSEPHSON JUNCTION

TRANSPORT PARAMETERS

Superconductor-topological insulator-superconductor Josephson junctions have been fabricated in order to study the width dependence of the critical current, normal state resistance and flux periodicity of the critical current modulation in an external field. Previous literature reports suggest anomalous scaling in topological junctions due to the presence of Majorana bound states. However, for most realised devices, one would expect that trivial $2 \pi$ periodic Andreev levels dominate transport. We also observe anomalous scaling behaviour of junction parameters, but the scaling can be well explained by mere geometric effects, such as the parallel bulk conductivity shunt and flux focusing.

\subsection{INTRODUCTION}

Topological insulator (TI) - superconductor (S) hybrids are potential systems for realizing $\mathrm{p}$-wave superconductivity and hosting Majorana zero-energy states [1-8]. The common singlet swave pairing from a nearby superconductor is predicted to induce a spinless $\mathrm{p}$-wave superconducting order parameter component in a topological insulator [4, 8] because of the spinmomentum locking of the surface states in a topological insulator [9-11]. In a Josephson junction between two s-waves superconductors with a topological insulator barrier (S-TI-S), Majorana Bound States (MBS) can occur with a $\sin (\phi / 2)$ currentphase relationship [4]. Contacts between superconductors and $3 \mathrm{D}$ topological insulators were realised on exfoliated flakes and

This chapter is accepted for publication in Superconductor Science and Technology (2014) as Geometric dependence of $\mathrm{Nb}-\mathrm{Bi}_{2} \mathrm{Te}_{3}-\mathrm{Nb}$ topological Josephson junction transport parameters by C. G. Molenaar, D. P. Leusink, X. L. Wang \& A. Brinkman. 
films of $\mathrm{Bi}_{2} \mathrm{Te}_{3}, \mathrm{Bi}_{2} \mathrm{Se}_{3}$, and strained $\mathrm{HgTe}$ [12-18], and the Josephson behaviour was investigated by measuring Fraunhofer patterns in the presence of an applied magnetic field and Shapiro steps due to microwave radiation $[13-15,17,18]$. Despite the presence of conductivity shunts through bulk TI, the Josephson current was found to be mainly carried by the topological surface states $[13,17,18]$.

Pecularities in the Fraunhofer diffraction patterns have been found for topological Josephson junctions [15, 19], including non-zero minima in the Fraunhofer patterns and periodicities which do not correspond to the junction size. In junctions with a varying width the characteristic energy $I_{C} R_{N}$ was reported to scale inversely with the junction width [15]. This observation has been phenomenologically attributed to the width dependence of the Majorana modes contributing to a highly distorted current-phase relationship [15]. The Majorana modes have also been held responsible for the unexpectedly small flux periodicities in the $I_{\mathcal{c}}(B)$ Fraunhofer pattern of the same junctions [15].

However, only one mode out of many channels is a MBS. For all non-perpendicular trajectories a gap appears in the Andreev Bound state spectra, giving trivial $2 \pi$ periodic bound states [20]. For typical device sizes fabricated so far, the number of channels is estimated to be large (a width of the order of a few 100 $\mathrm{nm}$ and a Fermi wavelength of the order of $k_{\mathrm{F}}^{-1}=1 \mathrm{~nm}$ already gives a few 100 modes). The Majorana signatures are, therefore, expected to be vanishingly small.

To understand the $I_{\mathcal{c}}(B)$ periodicity as well as the scaling of $I_{C} R_{N}$ with width, we have realised S-TI-S topological Josephson junctions with varying width. We also observe a non-trivial scaling of the critical current, normal state resistance and magnetic field modulation periodicity. However, a detailed analysis shows that all scaling effects can be explained by mere geometric effects of trivial modes. The dominance of trivial Andreev modes is supported by the absence of $4 \pi$ periodicity signatures in the Shapiro steps under microwave irradiation.

\subsection{EXPECTED MAJORANA RELATED MODIFICATIONS OF THE CRITICAL CURRENT MODULATION BY MAGNETIC FIELD AND MICROWAVES}

Screening external flux from a superconducting junction results in the characteristic Fraunhofer pattern in Josephson junctions due to the DC Josephson effect. The critical current is modu- 
lated by the magnetic flux with a periodicity of the superconducting flux quantum, $\Phi_{0}=\mathrm{h} / 2 \mathrm{e}$, threading the junction, due to the order-parameter being continuous around a closed contour. If the current-phase relationship is changed from $\sin (\phi)$ to $\sin (\phi / 2)$ in a topologically non-trivial junction the periodicity is expected to become $\mathrm{h} / \mathrm{e}$.

Additionally, for junctions where MBS are present it has been proposed that the minima in the Fraunhofer pattern are nonzero [21]. The current at the minima is predicted to be approximately equal to the supercurrent capacity of a single channel, $\mathrm{I}_{\mathrm{M}} \approx \Delta / \Phi_{0}$.

Applying an AC bias on top of the DC bias will create a frequency to voltage conversion, the AC Josephson effect. In the voltage state of the junction, at DC voltages equal to $k \Phi_{0} f=$ $\mathrm{khf} / 2 e$, with $\mathrm{k}$ an integer and $\mathrm{f}$ the frequency in $\mathrm{Hz}$, there will be a current plateau with zero differential resistivity at fixed finite voltages. The presence of these Shapiro steps in a superconducting junction is one of the hallmarks of the Josephson effect. In contrast to a Fraunhofer pattern, it does not depend on the geometry of the junction, but on the current-phase relationship of the junction. A sum of different current-phase relationships [22-27] I $=A_{1} \sin \phi / 2+A_{2} \sin \phi+A_{3} \sin 2 \phi+\ldots$ will result in current plateaus at $\mathrm{V}_{1, \mathrm{l}}=\mathrm{lhf} / \mathrm{e}, \mathrm{V}_{2, \mathrm{~m}}=\mathrm{mhf} / 2 \mathrm{e}, \mathrm{V}_{3, \mathrm{n}}=\mathrm{nhf} / 4 \mathrm{e}$, etc. For a pure $\sin (\phi / 2)$ relationship one expects steps only at $\mathrm{khf} / \mathrm{e}$. The actual width and the modulation as function of applied RF power of the current plateaus depends on the ratio between the applied RF frequency and the $I_{C} R_{N}$ product of the junctions. This can be numerically obtained by solving the Resistively Shunted Josephson (RSJ) [28] junction model.

\subsection{SAMPLE LAYOUT AND FABRICATION*}

Devices were designed with junctions of constant electrode separation and varying width. The fabrication is similar to the method used by Veldhorst et al. [13], but has been modified to reduce the number of fabrication steps and increase the number of usable devices available on one chip. Exfoliated flakes ${ }^{\dagger}$ are transferred to a $\mathrm{Si} / \mathrm{SiO}_{2}$ substrate. E-beam lithography, with $300 \mathrm{~nm}$ thick PMMA resist, is used to define junctions and contacts in two different write fields, eliminating the photo-lithography step.

${ }^{*}$ Device fabrication by D. P. Leusink.

${ }^{\dagger}$ Crystals grown by X. L. Wang. 


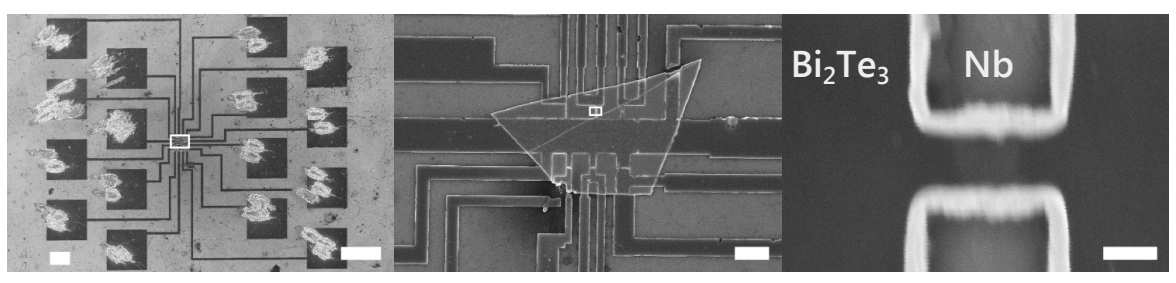

Figure 3.1: SCANNING ELECTRON MICROSCOPE IMAGES OF A TYPICAL DEVICE. The white bar is $200 \mu \mathrm{m}, 5 \mu \mathrm{m}$ and $100 \mathrm{~nm}$ wide in the three consecutive images respectively, and the white rectangles in the two left images mark the location of the image to the right. The first image show the $\mathrm{Nb}$ contact pads (dark grey) written with a large write field. The middle image shows the flake (trapezoid with bright edges and a step edge diagonal across) with leads (dark grey) leading to the junctions. The junctions are visible as faint white breaks in the leads. Along the top-row, left to right, are a 100,250 and $500 \mathrm{~nm}$ junction. On the bottom-row are a 750,1000 and $2000 \mathrm{~nm}$ junction. The $5 \mu \mathrm{m}$ on the right hand side of the flake is overexposed. The rightmost image is a close-up of a $250 \mathrm{~nm}$ by $150 \mathrm{~nm}$ designed junction.

In figure 3.1 the contact pads, written with a coarse write field, and the structure on the $\mathrm{Bi}_{2} \mathrm{Te}_{3}$ flake, written with a smaller and accurate write field, are visible. The smaller write field increases the resolution possible. An overlap of the structures was used in areas where the dose or write field was changed. These overlaps will cause overexposure and are only possible when the resolution is not critical. The $80 \mathrm{~nm}$ niobium superconducting film and a $2.5 \mathrm{~nm}$ capping layer of palladium are sputter deposited. The flake is Ar-ion etched at $50 \mathrm{eV}$ for I min prior to deposition resulting in transparent contacts.

The edge of the flake with the substrate provides a step edge for the $\mathrm{Nb}$ from the substrate to the flake, and it is advisable to keep the thickness of the flake comparable or less than the thickness of the $\mathrm{Nb}$ layer. The thickness of the sputter deposited $\mathrm{Nb}$ is limited by the thickness of the e-beam resist layer. For flakes of usable lateral dimensions, the thickness is generally in the order of $100 \mathrm{~nm}$. The contacts for the voltage and current leads are split on the flake: if a weak link occurs on the lead transition from flake to substrate this will not influence the measured current-voltage (IV) characteristic of the junction.

Structures with a disadvantageous aspect ratio (junction width to electrode separation), such as wider junctions, are prone to overexposure. This increases the risk of the junction ends 


\begin{tabular}{lll}
\hline JUNCTION & CRITICAL & NORMAL STATE \\
WIDTH $(\mathrm{nm})$ & CURRENT $(\mu \mathrm{A})$ & RESistanCE $(\Omega)$ \\
\hline 100 & 0.2 & 1.5 \\
250 & 3.5 & 1.14 \\
500 & 13.5 & 0.84 \\
1000 & 16 & 0.64 \\
\hline
\end{tabular}

Table 3.1: JUNCTION CHARACTERISTICS. The critical current $I_{C}$ and normal state resistance $R_{N}$ are given at $1.6 \mathrm{~K}$. The measured junction separation is $140 \mathrm{~nm}$. The $750 \mathrm{~nm}$ and $5000 \mathrm{~nm}$ wide junctions have shorted junctions due to e-beam overexposure, and the $2000 \mathrm{~nm}$ wide junction had a non-ohmic contact, caused by a break at the edge of the $\mathrm{Bi}_{2} \mathrm{Te}_{3}$ flake.

not being separated. For wider junctions a slightly larger separation has been used. Overexposure will decrease the actual separation. Actual dimensions are verified after fabrication as in figure 3.1.

The junctions are characterised in a pumped He cryostat with mu-metal screening and a superconducting $\mathrm{Nb}$ can surrounding the sample. The current and voltage leads are filtered with a two stage RC filter. A loop antenna for exposure to microwave radiation is pressed to the backside of the printed circuit board (PCB) holding the device. A coil perpendicular to the device surface is used to apply a perpendicular magnetic field. For different values of the applied microwave power or magnetic field current-voltage traces are recorded.

\subsection{MEASURED SCALING OF TRANSPORT PARAMETERS}

\subsubsection{Junction overview}

The devices are characterised by measuring their IV curves at $1.6 \mathrm{~K}$ under different magnetic fields and microwave powers. The microwave frequency of $6 \mathrm{GHz}$ is chosen for maximum coupling as determined by the maximum suppression of the supercurrent at the lowest power. The main measured junction parameters are given in table 3.1.

Both the magnetic and microwave field response has been studied for all junctions. Results for the 250, 500 and $1000 \mathrm{~nm}$ wide junctions are shown in figure 3.2. The supercurrent for 
the $100 \mathrm{~nm}$ wide junction was suppressed in a magnetic and microwave field without further modulation. In the response to the microwave field a sharp feature is visible starting at $200 \mu \mathrm{A}$ and $-10 \mathrm{dBm}$ for the $1000 \mathrm{~nm}$ junction. This is likely the result of an unidentified weak link in one of the leads. The fainter structures in the $250 \mathrm{~nm}$ junction starting at 43 and $60 \mu \mathrm{A}$ and $-40 \mathrm{dBm}$ are reminiscent of an echo structure described by Yang et al. [14] for $\mathrm{Pb}-\mathrm{Bi}_{2} \mathrm{Se}_{3}-\mathrm{Pb}$ Josephson junctions. Measuring the microwave response at the minima of the Fraunhofer pattern [21] yielded no Shapiro features.

\subsubsection{Scaling of $\mathrm{I}_{C}$ and $\mathrm{R}_{N}$}

In general, the normal state resistance, $\mathrm{R}_{\mathrm{N}}$, of a lateral SNS junction $[29,30]$ is expected to scale inversely with junction width, whereas $I_{c}$ is expected to be proportional to the width, such that the $I_{C} R_{N}$ product is constant [28]. Josephson junctions on topological insulators are similar to SNS junctions with an induced proximity effect by superconducting leads into a TI surface state. For junctions on $\mathrm{Bi}_{2} \mathrm{Te}_{3}$ the transport was found to be in the clean limit, with a finite barrier at the interface between the superconductor and the surface states [13]. The supercurrent for ballistic SNS junctions with arbitrary length and barrier transparency is given by [31], which was found to fit the data of Veldhorst et al. well [13]. The normal state resistance in $\mathrm{Bi}_{2} \mathrm{Te}_{3}$ is complicated by the diffusive bulk providing an intrinsic shunt. The leads on the $\mathrm{Bi}_{2} \mathrm{Te}_{3}$ flake leading up to the junction also contribute towards a normal state conductivity shunt without carrying supercurrent. This results in current paths not only directly between the two electrodes but also through and across the whole area of the flake to the left and right of the electrodes.

The scaling of $I_{c}$ and $R_{N}$ with junction width is shown in Figure 3.3. In the junctions with an aspect ratio (width of the junction divided by electrode separation) greater than 5 , the $I_{C} R_{N}$ product is approximately $11 \mu \mathrm{V}$, similar to junctions where the the length was varied instead of the width $[13,32]$. Below this, the $I_{C} R_{N}$ product falls sharply. To verify whether this can be due to Majorana modes we estimate the number of conducting channels in these small junctions. The number of channels in a junction is related to the width of the junction: $M=\frac{k_{F} \times W}{\pi}{ }^{1}$.

\footnotetext{
${ }^{1}$ The wave vector for linear dispersion is given by $k_{\mathrm{F}}=\frac{E_{\mathrm{F}}}{\hbar v_{\mathrm{F}}} \approx$ $2 \times 10^{9} \mathrm{~m}^{-1}$. The Fermi energy is taken as $150 \mathrm{meV}$ and the Fermi velocity is in the order of $1 \times 10^{5} \mathrm{~m} / \mathrm{s}$.
} 


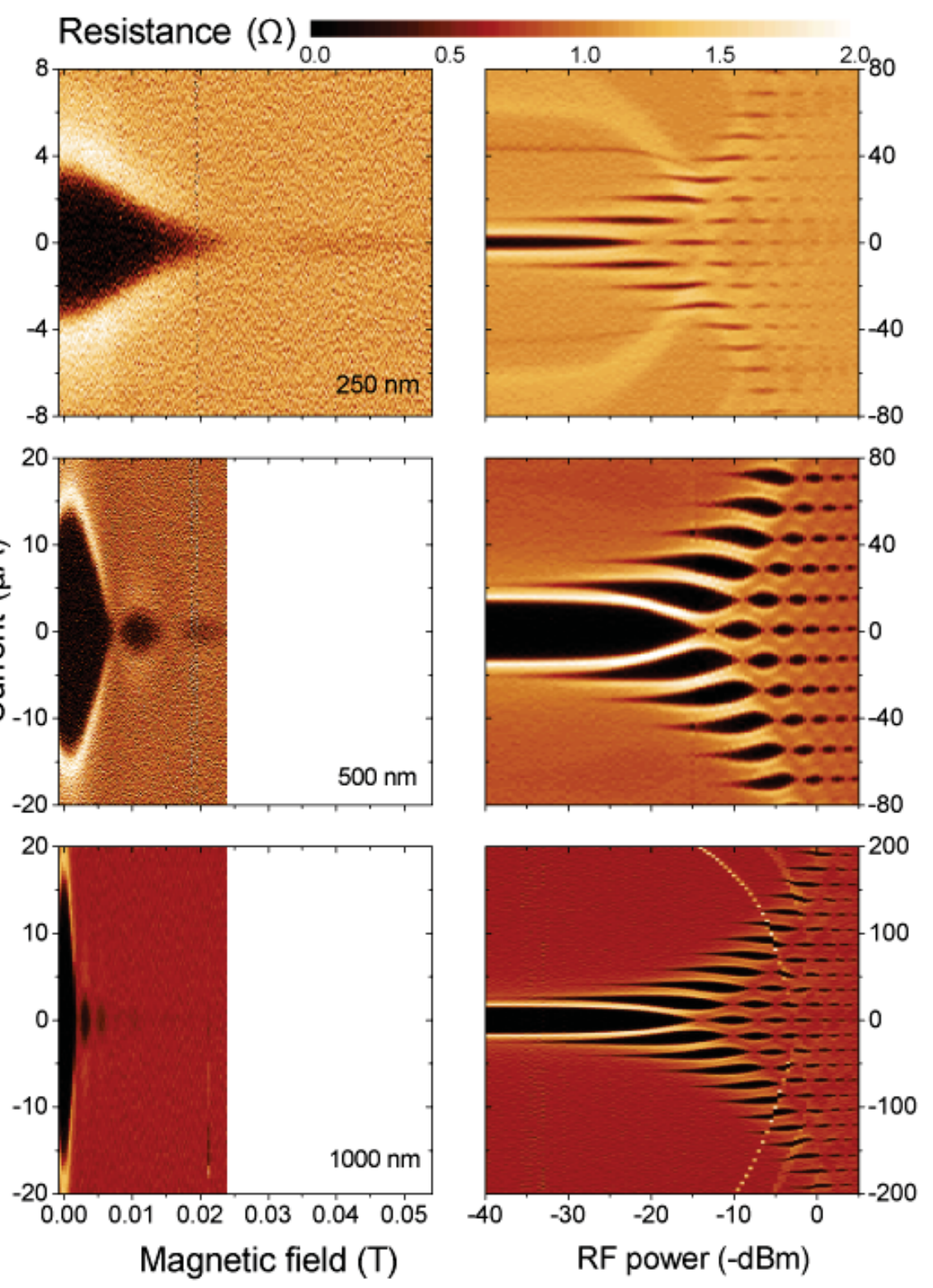

Figure 3.2: MAGNETIC FIELD AND MiCROWAVE POWER DEPENDENCE. The top row figures show the dynamic resistance of the $250 \mathrm{~nm}$ wide junction, the middle row figures correspond to the $500 \mathrm{~nm}$ wide junction and the bottom row figure to the $1000 \mathrm{~nm}$ wide junction. The left column shows the reaction to an applied magnetic field, the right column the reaction to microwave power at $6 \mathrm{GHz}$. The horizontal line at $\sim 19 \mathrm{mT}$ is an artifact of the magnet current source. IV curves for these junctions are shown in figure 3.4. 

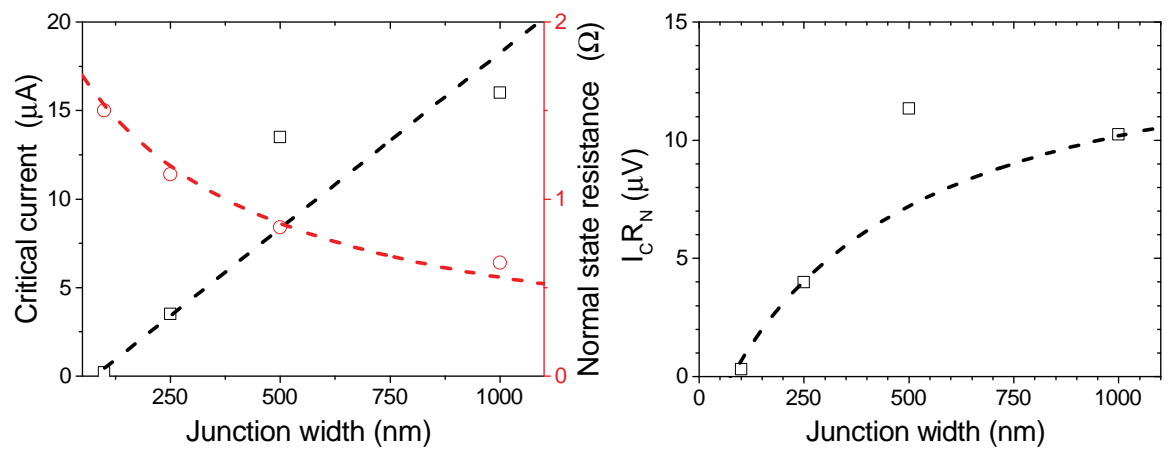

Figure 3.3: SCALING OF THE CRITICAL CURRENT, NORMAL RESISTANCE AND $I_{C} R_{N}$ PRODUCT. In the left panel the measured critical currents (black squares) and normal state resistances (red circles) are plotted. The critical current is assumed to be linear (black dashes). The resistance is modelled as a width resistivity $\rho_{w}=7.9 \times 10^{-7} \Omega \mathrm{m}$ in parallel with a constant shunt resistance $R_{\text {parallel }}=1.9 \Omega$. The $I_{C} R_{N}$ product is plotted in the right panel, with the dashed line as the product of the fits in the left panel. The fit approaches the $\mathrm{I}_{\mathrm{C}} \mathrm{R}_{\mathrm{N}}$ product of 10 to $15 \mu \mathrm{V}$ found in junctions of varying width [13].

For a $100 \mathrm{~nm}$ junction this means that there are more than 60 channels active in the junction, and a MBS will not dominate transport properties.

Rather, due to the open edges of the junctions, the scaling of the normal state resistance is not directly proportional to the junction width, but offset due to the whole flake providing a current shunt. This is similar to an infinite resistor network [33] providing a parallel resistance to the resistance due to the separation of the two leads. Taking this into account, the resistance between the two leads takes the form $R=\left(\rho_{W} \times\right.$ $\left.R_{\text {parallel }}\right) /\left(W \times R_{\text {parallel }}+\rho_{W}\right)$, where $r o_{W} / W$ gives the junction resistance without a current shunt and $R_{\text {parallel }}$ is the resistance due to the current shunt through the flake. This equation does not disentangle the surface and bulk contributions but treats them as scaling the same. In the zero-width limit, the resistance is cut-off and does not diverge to infinity. The resulting $I_{C} R_{N}$ product can then be well explained by the scaling of $R_{N}$ (including the shunt) and the usual scaling of $I_{c}$ with width $\left(I_{c}\right.$ being directly proportional to the number of channels, given by the width of the junctions with respect to the Fermi wavelength). Note, that the expected scaling of $I_{c}$ contrasts previous observations of inverse scaling [15]. 


\subsubsection{Periodicity in $\Phi_{0}$}

The critical current of Josephson junctions oscillate in an applied magnetic field due to a phase difference induced across the junction. The magnetic flux in the junction area is the product of the area of weak superconductivity between the two electrodes and flux density in this area. The area of the junction is given by $W \times\left(l+2 \lambda_{L}\right)$, where $W, l$ and $\lambda_{L}$ are the width, length and London penetration depth respectively. The investigated junctions are smaller than or comparable to the Josephson penetration depth, $\lambda_{J}=\sqrt{\Phi_{0} /\left(2 \pi \mu_{0} d^{\prime} J_{C}\right)}$, where $d^{\prime}$ is the largest dimension (corrected by the London penetration depth) of the junction and $\mathrm{J}_{C}$ has been estimated using the bulk mean free path of $\mathrm{Bi}_{2} \mathrm{Te}_{3}$ crystals, $22 \mathrm{~nm} \mathrm{[13],} \mathrm{which} \mathrm{allows} \mathrm{us} \mathrm{to} \mathrm{ig-}$ nore the field produced by the Josephson current. For the $80 \mathrm{~nm}$ thick $\mathrm{Nb}$ film used we use the bulk London penetration depth, $39 \mathrm{~nm}[34,35]$.

The superconducting leads may be regarded as perfect diamagnets. This leads to flux lines being diverted around the superconducting structure. This causes flux focussing in the junctions, as more flux lines pass through the junctions due to their expulsion from the superconducting bulk. We estimate the amount of flux focussing by considering the shortest distance a flux line has to be diverted to not pass through the superconducting lead. In a long lead, half are passed to the one side and half to the other side. At the end of the lead the flux lines are diverted into the junction area. The flux diverted is $\left(\left(W / 2-\lambda_{L}\right)^{2} \times B\right.$, see also the inset of Figure 3.2. This occurs at both electrodes, and is effectively the same as increasing the junction area by $2 \times\left(\mathrm{W} / 2-\lambda_{\mathrm{L}}\right)^{2}$. Without flux focussing, the expected magnetic field periodicity is given by the dashed line in figure 3.4(a). Correcting for flux focussing and taking $\lambda_{\mathrm{L}}=39 \mathrm{~nm}$ results in the solid line, closely describing the measured periods.

The colour graphs in figure 3.2 show the modulation of the critical current with microwave power. In figure 3.4(b) IV traces for different applied powers are plotted. The steps all occur at multiples of $\Phi_{0} f=12.4 \mu \mathrm{V}$. A $4 \pi$ periodic Josephson effect will result in steps only at even multiples of $\Phi_{0}$ f. Shapiro steps are not geometry dependent: in combination with the previously introduced geometry corrected magnetic field periodicity this illustrates the $2 \pi$ periodic Josephson effect in these junctions. 

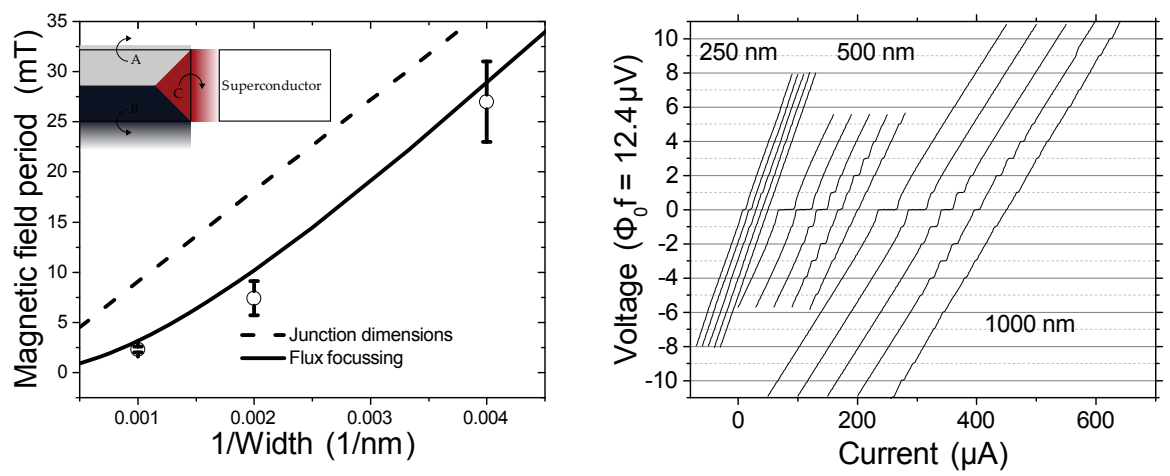

Figure 3.4: BEHAVIOUR OF FrAUNHOFER OSCILLATION FREQUENCY AND SHAPIRO STEPS. In the left panel the modulation period of the Josephson current as a function of the external field is plotted as a function of the inverse junction width. The dashed line is the expected period for a rectangular junction. The solid line takes into account flux focussing, as presented in the inset. The flux incident on the grey areas $A$ and $B$ is diverted to the sides of the junction lead, while the red area $C$ is added to the effective junction area between the two leads. The dimensions of the superconductor have been reduced by the London penetration depth, since flux can penetrate this area. The right panel shows IV characteristics under $6 \mathrm{GHz}$ microwave irradiation. The line graphs are at $-40,-30,-20,-10$ and o dBm powers for the 250, 500 and $1000 \mathrm{~nm}$ wide junctions, and are offset in current for clarity. All current plateaus are at multiples of $\Phi_{0} f=12.4 \mu \mathrm{V}$. 


\section{$3 \cdot 5$ CONCLUSION}

We investigated superconducting junctions, coupling $\mathrm{Nb}$ leads on the surface of a $\mathrm{Bi}_{2} \mathrm{Te}_{3}$ flake, by varying the junction width. The critical current and normal state resistance decrease and increase respectively with reduced junction width. However, the $I_{C} R_{N}$ product is found to be geometry dependent, as the normal state resistance does not diverge for zero width. The decreasing $\mathrm{I}_{\mathrm{C}} \mathrm{R}_{\mathrm{N}}$ product with reduced junction width is understood when taking into account the resistance due to the entire flake surface. The $\mathrm{I}_{C} \mathrm{R}_{\mathrm{N}}$ product becomes of the order of 10 to $15 \mu \mathrm{V}$ for wide junctions, similar to previous junctions [32]. The junctions are found to be periodic with $\Phi_{0}$ in a magnetic field when flux focussing is taken into account. Microwave irradiation results in steps at voltages at $k \Phi_{0} f$, which is to be expected from junctions with ten to hundred conducting channels contributing to the coupling between the superconducting leads.

Using topological insulators with reduced bulk conductivity should result in increased $\mathrm{I}_{\mathrm{C}} \mathrm{R}_{\mathrm{N}}$ products and allow for electrostatic control of the Fermi energy. With similar junction geometries this will allow for reduction and control of the number of superconducting channels. This step will allow the behaviour

of a possible MBS to be uncovered and separated from geometric effects which affect all conducting channels in S-TI-S junctions.

\section{BIBLIOGRAPHY}

[1] N. Read and D. Green, Phys. Rev. B 61, 10267 (2000).

[2] A. Y. Kitaev, Physics-Uspekhi 44, 131 (2001).

[3] D. A. Ivanov, Phys. Rev. Lett. 86, 268 (2001).

[4] L. Fu and C. L. Kane, Phys. Rev. Lett. 100, 096407 (2008).

[5] J. Nilsson, A. R. Akhmerov, and C. W. J. Beenakker, Phys. Rev. Lett. 101, 120403 (2008).

[6] Y. Tanaka, T. Yokoyama, and N. Nagaosa, Phys. Rev. Lett. 103, 107002 (2009).

[7] T. D. Stanescu, J. D. Sau, R. M. Lutchyn, and S. Das Sarma, Phys. Rev. B 81, 241310 (2010).

[8] A. C. Potter and P. A. Lee, Phys. Rev. B 83, 184520 (2011). 
[9] D. Hsieh, Y. Xia, D. Qian, L. Wray, J. H. Dil, F. Meier, J. Osterwalder, L. Patthey, J. G. Checkelsky, N. P. Ong, A. V. Fedorov, et al., Nature 460, 1101 (2009).

[10] Y. L. Chen, J. G. Analytis, J.-H. Chu, Z. K. Liu, S.-K. Mo, X. L. Qi, H. J. Zhang, D. H. Lu, X. Dai, Z. Fang, S. C. Zhang, et al., Science 325, 178 (2009).

[11] M. Z. Hasan and C. L. Kane, Rev. Mod. Phys. 82, 3045 (2010).

[12] B. Sacépé, J. B. Oostinga, J. Li, A. Ubaldini, N. J. Couto, E. Giannini, and A. F. Morpurgo, Nat Communications 2, 575 (2011).

[13] M. Veldhorst, M. Snelder, M. Hoek, T. Gang, V. K. Guduru, X. L. Wang, U. Zeitler, W. G. van der Wiel, A. A. Golubov, H. Hilgenkamp, and A. Brinkman, Nature Materials 11, 417 (2012).

[14] F. Yang, F. Qu, J. Shen, Y. Ding, J. Chen, Z. Ji, G. Liu, J. Fan, C. Yang, L. Fu, and L. Lu, Phys. Rev. B 86, 134504 (2012).

[15] J. R. Williams, A. J. Bestwick, P. Gallagher, S. S. Hong, Y. Cui, A. S. Bleich, J. G. Analytis, I. R. Fisher, and D. Goldhaber-Gordon, Phys. Rev. Lett. 109, 056803 (2012).

[16] J. B. Oostinga, L. Maier, P. Schüffelgen, D. Knott, C. Ames, C. Brüne, G. Tkachov, H. Buhmann, and L. W. Molenkamp, Phys. Rev. X 3, 021007 (2013).

[17] S. Cho, B. Dellabetta, A. Yang, J. Schneeloch, Z. Xu, T. Valla, G. Gu, M. J. Gilbert, and N. Mason, Nat Commun 4, 1689 (2013).

[18] L. Galletti, S. Charpentier, M. Iavarone, P. Lucignano, D. Massarotti, R. Arpaia, Y. Suzuki, K. Kadowaki, T. Bauch, A. Tagliacozzo, F. Tafuri, et al., Phys. Rev. B 89, 134512 (2014).

[19] C. Kurter, A. D. K. Finck, P. Ghaemi, Y. S. Hor, and D. J. Van Harlingen, Phys. Rev. B 90, 014501 (2014).

[20] M. Snelder, M. Veldhorst, A. A. Golubov, and A. Brinkman, Phys. Rev. B 87, 104507 (2013).

[21] A. C. Potter and L. Fu, Phys. Rev. B 88, 121109 (2013). 
[22] H.-J. Kwon, K. Sengupta, and V. Yakovenko, Brazilian Journal of Physics 33, 653 (2003).

[23] H.-J. Kwon, K. Sengupta, and V. M. Yakovenko, The European Physical Journal B-Condensed Matter and Complex Systems 37, 349 (2004).

[24] H.-J. Kwon, V. Yakovenko, and K. Sengupta, Low temperature physics 30, 613_1 (2004).

[25] L. Fu and C. L. Kane, Phys. Rev. B 79, 161408 (2009).

[26] P. A. Ioselevich and M. V. Feigel'man, Phys. Rev. Lett. 106, 077003 (2011).

[27] A. Pal, Z. Barber, J. Robinson, and M. Blamire, Nat Commun 5 , (2014).

[28] M. Tinkham, Introduction to Superconductiviy (Dover Publications, 2004), 2nd ed.

[29] K. K. Likharev, Rev. Mod. Phys. 51, 101 (1979).

[30] A. A. Golubov, M. Y. Kupriyanov, and E. Il'ichev, Rev. Mod. Phys. 76, 411 (2004).

[31] A. V. Galaktionov and A. D. Zaikin, Phys. Rev. B 65, 184507 (2002).

[32] M. Veldhorst, C. G. Molenaar, X. L. Wang, H. Hilgenkamp, and A. Brinkman, Applied Physics Letters 100, 072602 (2012).

[33] J. Cserti, American Journal of Physics 68, 896 (2000).

[34] A. I. Gubin, K. S. Il'in, S. A. Vitusevich, M. Siegel, and N. Klein, Phys. Rev. B 72, 064503 (2005).

[35] B. W. Maxfield and W. L. McLean, Phys. Rev. 139, A1515 (1965). 

A Josephson supercurrent has been induced into the three-dimensional topological insulator $\mathrm{Bi}_{1.5} \mathrm{Sb}_{0.5} \mathrm{Te}_{1.7}$ $\mathrm{Se}_{1.3}$. We show that the transport in $\mathrm{Bi}_{1.5} \mathrm{Sb}_{0.5} \mathrm{Te}_{1.7^{-}}$ $\mathrm{Se}_{1.3}$ exfoliated flakes is dominated by surface states and that the bulk conductivity can be neglected at the temperatures where we study the proximity induced superconductivity. We prepared Josephson junctions with widths in the order of $40 \mathrm{~nm}$ and lengths in the order of 50 to $80 \mathrm{~nm}$ on several $\mathrm{Bi}_{1.5} \mathrm{Sb}_{0.5^{-}}$ $\mathrm{Te}_{1.7} \mathrm{Se}_{1.3}$ flakes and measured down to $30 \mathrm{mK}$. The Fraunhofer patterns unequivocally reveal that the supercurrent is a Josephson supercurrent. The measured critical currents are reproducibly observed on different devices and upon multiple cooldowns, and the critical current dependence on temperature as well as magnetic field can be well explained by diffusive transport models and geometric effects.

Topological insulators (TIs) have conducting surface states with a locking between the electron momentum and its spin [1-9]. Besides bearing promise for high temperature spintronic applications [10-12], TIs are also candidate materials to host exotic superconductivity. For example, $p+i p$ order parameter components [13, 14] and Majorana zero energy states [15-18] have been theoretically predicted. The topological superconductivity can either be intrinsic [19] or proximised by a nearby superconductor [20-22].

This chapter is accepted for publication in Superconductor Science and Technology (2014) as Josephson supercurrent in a topological insulator without a bulk shunt by M. Snelder, C. G. Molenaar, Y. Pan, Y. K. Huang, A. de Visser, A. A. Golubov, W.G. van der Wiel, H. Hilgenkamp, M.S. Golden \& A. Brinkman.

The author of this PhD thesis has contributed to this manuscript by the measurements presented in figures 4.2 and 4.3 , the analysis and interpretation of the data and by contributing in the writing of the manuscript. 
The first generation of topological insulators, Bi-based materials such as $\mathrm{Bi}_{1-\chi} \mathrm{Sb}_{\chi}$ alloys, and later $\mathrm{Bi}_{2} \mathrm{Te}_{3}$ and $\mathrm{Bi}_{2} \mathrm{Se}_{3}$ compounds, exhibit topological surface states but also have an additional shunt from the conducting bulk, mainly due to anti-site defects and vacancies [5, 23, 24]. Josephson junctions [22, 25-35] and SQUIDs $[27,34-37]$ have been realised in these topological surface states, but the practical use of these topological devices is limited by the bulk shunt $[36,38]$. Secondary and ternary compounds have been engineered to increase the bulk resistance and increase the stability of the surface states. The most promising examples of the latest generation three-dimensional TIs are $\mathrm{Bi}_{2-\chi} \mathrm{Sb}_{\chi} \mathrm{Te}_{3-y} \mathrm{Se}_{y}$ [39] and strained $\mathrm{HgTe}$ [40-42].

In this work we report the realisation of a Josephson supercurrent across $50 \mathrm{~nm}$ of the topological insulator $\mathrm{Bi}_{1.5} \mathrm{Sb}_{0.5} \mathrm{Te}_{1.7^{-}}$ $\mathrm{Se}_{1.3}$. We first show that in our $\mathrm{Bi}_{1.5} \mathrm{Sb}_{0.5} \mathrm{Te}_{1.7} \mathrm{Se}_{1.3}$ no bulk conduction is present at low temperatures and that the observed surface states are of a topologically non-trivial nature. We then demonstrate Josephson junction behaviour reproducibly on different flakes and during multiple cooldowns. The width of the superconducting $\mathrm{Nb}$ electrodes is very narrow, of the order of $40 \mathrm{~nm}$, anticipating future work on topological devices with only a few modes [43-45].

\subsection{TRANSPORT PROPERTIES OF EXFOLIATED BI $1.5 \mathrm{SB}_{0.5} \mathrm{TE}_{1.7-}$ SE1.3 FLAKES}

$\mathrm{Bi}_{1.5} \mathrm{Sb}_{0.5} \mathrm{Te}_{1.7} \mathrm{Se}_{1.3}$ single crystals were obtained by melting stoichiometric amounts of the high purity elements Bi (99.999 $\%), \mathrm{Sb}(99.9999 \%)$, Te (99.9999\%) and Se (99.9995\%). The raw materials were sealed in an evacuated quartz tube which was vertically placed in the uniform temperature zone of a box furnace to ensure the homogeneity of the batch. The molten material was kept at $850{ }^{\circ} \mathrm{C}$ for 3 days and then cooled down to $520{ }^{\circ} \mathrm{C}$ with a speed of $3{ }^{\circ} \mathrm{C} / \mathrm{h}$. Next, the batch was annealed at $520{ }^{\circ} \mathrm{C}$ for 3 days, followed by cooling to room temperature at a speed of $10{ }^{\circ} \mathrm{C} / \mathrm{min}[46]$.

Smooth flakes are prepared using mechanical exfoliation on a silicon-on-insulator substrate. To determine the transport characteristics of $\mathrm{Bi}_{1.5} \mathrm{Sb}_{0.5} \mathrm{Te}_{1.7} \mathrm{Se}_{1.3}$, Hall bars are prepared using e-beam lithography and argon ion etching on exfoliated flakes with a thickness ranging from 80 till $200 \mathrm{~nm}$. Au electrodes are

\footnotetext{
*Crystals grown by Y. Pan, Y. K. Huang, A. de Visser \& M. S. Golden
} 
defined by photolithography and lift-off. During all the fabrication steps the Hall bar is covered with either e-beam resist or photoresist protecting the surface from damage or contamination. The Hall bars are $7 \mu \mathrm{m}$ long and $700 \mathrm{~nm}$ wide. Figure 4.I(a) shows a SEM image of such a Hall bar.

A typical temperature dependence of the resistance of the Hall bars is shown in Fig. 4.I(b). At high temperature, the crystal exhibits semiconductor-like thermally activated behaviour. Below $150 \mathrm{~K}$ the resistance stabilises, indicating metallic surface channels. At high temperatures the transport properties are determined by the bulk of the crystal, while at low temperatures the surfaces provide the dominant charge carriers. To verify this, the high temperature part of the curve is modelled as a semiconductor using $R \propto e^{\Delta / k_{B} T}$. The best fit between 200 $\mathrm{K}$ and $300 \mathrm{~K}$ gives $\Delta=18 \mathrm{meV}$. This means that the Fermi energy is positioned $18 \mathrm{meV}$ below the bottom of the conduction band. The entire bulk band gap would be larger than 18 $\mathrm{meV}$. At $300 \mathrm{~K}$, the bulk contribution is dominant and allows for a one-band interpretation of the Hall effect measurement (see Fig. 4.I(c)) at this temperature, giving a bulk carrier density of ${ }_{10}^{17} \mathrm{~cm}^{-3}$. Extrapolating the carrier freeze out to low temperatures we find a negligible bulk conduction at low temperatures. At low temperatures all transport is due to the surface states. Hall and longitudinal resistance measurements at $2 \mathrm{~K}$ are used to determine the electron density and mobility of the surface states. We reproducibly obtain surface electron densities in the range of $10^{12}-10^{13} \mathrm{~cm}^{-2}$ with mobilities between 120 and 450 $\mathrm{cm}^{2} / \mathrm{Vs}$. The resulting mean free path of the order of 10 to 40 $\mathrm{nm}$ is comparable to the mean free path of the electron-like surface states found by Taskin et al. [39].

Figure 4.I (d) shows the change in longitudinal conductance as function of applied perpendicular magnetic field. To verify the topological character of the surface states the HikamiLarkin-Nagaoka theory [47] is used to fit the low-field magnetoconductance in perpendicular field,

$$
\begin{aligned}
& \Delta \mathrm{G}_{\square}\left(\mathrm{B}_{\perp}\right)-\Delta \mathrm{G}_{\square}(0)= \\
& \alpha \frac{e^{2}}{2 \pi^{2} \hbar}\left[\psi\left(\frac{1}{2}+\frac{\hbar}{4 e l_{\phi}^{2} \mathrm{~B}_{\perp}}\right)-\ln \left(\frac{\hbar}{4 e l_{\phi}^{2} \mathrm{~B}_{\perp}}\right)\right],
\end{aligned}
$$

where $G_{\square}=\frac{L}{W R}$ with $L$ being the length and $W$ the width of the Hall bar, $R$ is the measured resistance, $\psi$ is the digamma

\footnotetext{
${ }^{\dagger}$ Bulk characterisation and device fabrication by M. Snelder.
} 
function, $l_{\phi}$ is the inelastic scattering length, $\alpha$ is a parameter indicating the strength of the spin-orbit interaction and $\mathrm{B}_{\perp}$ is the perpendicular magnetic field. If the spin-orbit interaction is weak, a positive value of $\alpha=1$ is expected, as opposed to strong spin-orbit interaction where a negative value of -0.5 is expected [47]. Due to the chiral-spin texture of a topological insulator and the contribution of both top and bottom surfaces in the transport measurements, an $\alpha$ parameter of -1 is expected. In Fig. 4.I(d) the magnetoconductance is fitted with $l_{\phi}=144$ $\mathrm{nm}$ and $\alpha=-1.01$ which would be in good agreement with the presence of just the bottom and top topologically non-trivial surface states.

However, we cannot rule out the presence of additional trivial two-dimensional metallic surface states that could originate from surface band bending. The possibility of the conduction band bending down below the Fermi energy has been observed in $\mathrm{Bi}_{1.5} \mathrm{Sb}_{0.5} \mathrm{Te}_{1.7} \mathrm{Se}_{1.3}$ [39]. Angle resolved photoemission spectroscopy has revealed that the presence of these non-topological surface states is depending on surface absorbents and could vary over time [48].

\subsection{JUNCTION FABRICATION}

Now that we have verified the topological nature of our crystals we turn to fabricating Josephson junctions with $\mathrm{Bi}_{1.5} \mathrm{Sb}_{0.5} \mathrm{Te}_{1.7^{-}}$ $\mathrm{Se}_{1.3}$ as the weak link. Transferred similarly to the devices for determining the transport properties, smooth flakes on $\mathrm{Si} / \mathrm{SiO}_{2}$ substrates are used for devices. E-beam lithography is used to define the junctions and the contact pads. Thereafter we perform a 30 second low voltage etching step to avoid large damage to the surface followed by sputtering in-situ $25 \mathrm{~nm} \mathrm{Nb}$ and $2.5 \mathrm{~nm} \mathrm{Pd}$ to protect the $\mathrm{Nb}$ layer, and lift-off of the excess material. The resulting $\mathrm{Nb} / \mathrm{Bi}_{1.5} \mathrm{Sb}_{0.5} \mathrm{Te}_{1.7} \mathrm{Se}_{1.3} / \mathrm{Nb}$ junction has been covered by photoresist during the entire process and no damage from etching or growth has occurred to the crystal surface between the electrodes. In figure 4.2(a) a junction is visible in a SEM image, and similar junctions have been prepared on different flakes. The width is about $40 \mathrm{~nm}$ and the electrode separation is about $50 \mathrm{~nm}$. The motivation for the junction length is based on our earlier work on $\mathrm{Bi}_{2} \mathrm{Te}_{3}$ with junctions ranging from 50-250 $\mathrm{nm}$ [25]. The mean free path in $\mathrm{Bi}_{1.5} \mathrm{Sb}_{0.5} \mathrm{Te}_{1.7} \mathrm{Se}_{1.3}$ is ten times smaller compared to $\mathrm{Bi}_{2} \mathrm{Te}_{3}$ which implies that the junction length should also be reduced by a factor of ten to 
(a)

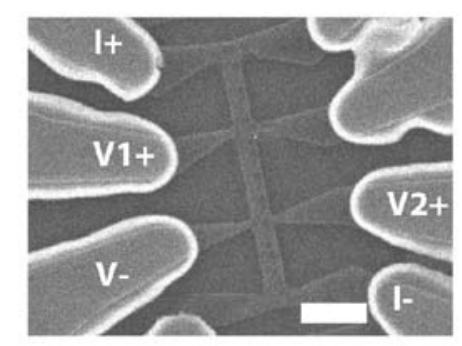

(c)

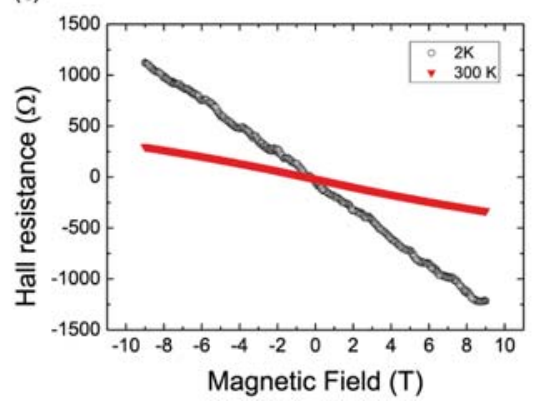

(b)

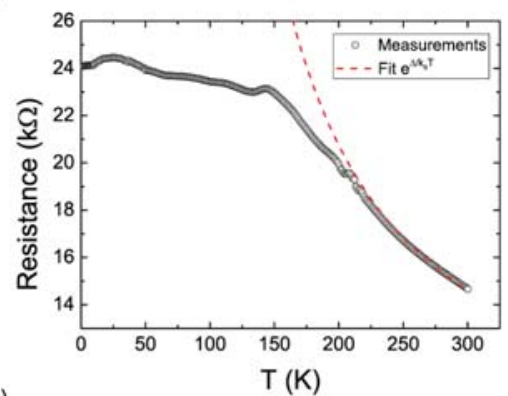

(d)

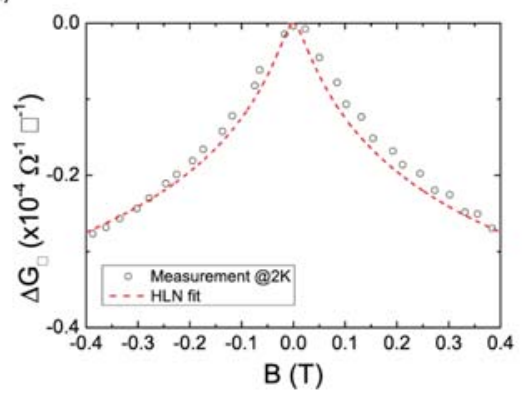

Figure 4.1: FLAKE CHARACTERISTICS. (a) Scanning electron microscopy image of a Hall bar of $\mathrm{Bi}_{1.5} \mathrm{Sb}_{0.5} \mathrm{Te}_{1.7} \mathrm{Se}_{1.3}$. The dimension of this Hall bar is different from the Hall bar of the measurements shown in Fig. 4.I (c-d). The Hall bar of the measurements has a width of $560 \mathrm{~nm}$ and a length of $6.8 \mu \mathrm{m}$. The dark grey part is etched away. The light gray part between the electrodes is the topological insulator. The white scale bar is $5 \mu \mathrm{m}$. The longitudinal resistance is measured between $\mathrm{V}_{1}+$ and $\mathrm{V}_{-}$, and the Hall resistance between $\mathrm{V}$ and $\mathrm{V} 2+$. (b) Typical temperature dependence of the resistance of an exfoliated flake of $\mathrm{Bi}_{1.5} \mathrm{Sb}_{0.5} \mathrm{Te}_{1.7} \mathrm{Se}_{1.3}$, measured in a Hall bar configuration. The dependence is fitted with a function $e^{\Delta / k_{B} T}$.(c) A typical Hall effect measurement at $2 \mathrm{~K}$ (black) and $300 \mathrm{~K}$ (red). The negative slope implies that the charge carriers are electrons. (d) Measured magnetoconductance, together with a fit of the HLN theory to the data. The best fit is obtained for $\alpha=-1.01$ and $l_{\phi}=144 \mathrm{~nm}$. 
(a)

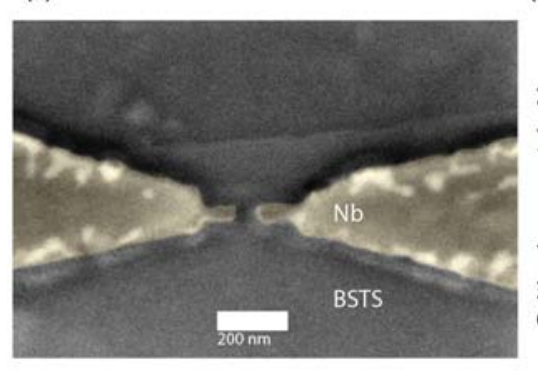

(b)

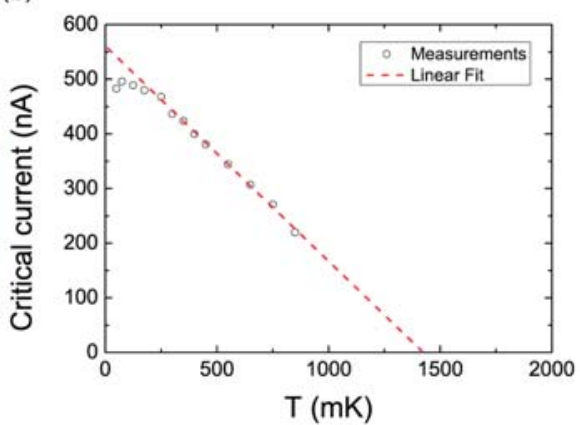

Figure 4.2: JUNCTION AND NANOWIRE. (a) Scanning electron microscopy image of a $\mathrm{Nb} / \mathrm{Bi}_{1.5} \mathrm{Sb}_{0.5} \mathrm{Te}_{1.7} \mathrm{Se}_{1.3} / \mathrm{Nb}$ junction. The $\mathrm{Nb}$ layer has been coloured yellow for clarity. The junction is $57 \mathrm{~nm}$ long and $38 \mathrm{~nm}$ wide. (b) The dependence of the critical current of the $\mathrm{Nb}$ nanowire as function of temperature. From the linear fit to the data points in the range of $300-850 \mathrm{mK}$, the critical temperature is extrapolated to be $1.4 \mathrm{~K}$. Close to the transition temperature, the gap and therefore the critical current can be approached by a linear dependence [49].

realise sufficient coupling between the electrodes. Simultaneously, this design takes a step forward towards experiments for the observation of a quantised supercurrent where only a few modes should be present [43-45]. In order to achieve the presence of a few modes, the junction width should be of the order of the Fermi wave length. For a $40 \mathrm{~nm}$ wide junction and a Dirac velocity of $4.5 \times 10^{5} \mathrm{~m} / \mathrm{s}$ this means that the Fermi level would have to be tuned within $50 \mathrm{meV}$ from the Dirac point by means of gating, which is quite reasonable. To realise these dimensions a thin layer of PMMA $(80 \mathrm{~nm})$ is used which limits the thickness of $\mathrm{Nb}$ that could be grown. The reduction of the dimensions of the $\mathrm{Nb}$ leads reduces the gap of $\mathrm{Nb}$. A $40 \mathrm{~nm}$ wide and $250 \mathrm{~nm}$ long $\mathrm{Nb}$ nanowire is prepared in the same run to determine the properties of the niobium with these dimensions. In figure 4.2(b) the critical current of this nanowire as a function of temperature is presented, and a reduction of the critical temperature from $9 \mathrm{~K}$ of the $\mathrm{Nb}$ of our thin film process to about $1.4 \mathrm{~K}$ is visible.

\subsection{RESULts}

Measurements are performed in a cryogen free dilution refrigerator with low pass filtering of current and voltage signals 
using pi filters, printed circuit board copper powder filters [50], and RC filters. We measured the critical current as function of temperature and the modulation of the critical current by an applied magnetic field at $30 \mathrm{mK}$. We will start this section with a discussion of the temperature dependence followed by an analysis of the Fraunhofer pattern.

\subsubsection{Temperature dependence of the critical current}

At $30 \mathrm{mK}$ we observe a critical current of $14 \mathrm{nA}$ for a junction with $57 \mathrm{~nm}$ electrode separation, visible in figure 4.2(a), and $4.8 \mathrm{nA}$ for a $80 \mathrm{~nm}$ long junction. Using the extracted critical temperature of the $\mathrm{Nb}$ nanowire as the transition temperature for the leads, the normalised temperature dependence of the critical current is plotted in figure 4.3(a) for the $57 \mathrm{~nm}$ junction. The boundary between Josephson coupling and thermal noise $\left(\hbar \mathrm{I}_{0} / e\right) /\left(\mathrm{k}_{\mathrm{B}} \mathrm{T}\right)=1$ is illustrated with the dashed line. In figure $4.3(\mathrm{~b})$ the $\mathrm{dV} / \mathrm{dI}$ characteristics around the thermal limit reveal indeed that a zero resistance state and coherence peaks are only visible below the thermal limit. The coherence peaks are used for determining the critical current below the thermal limit. Above the thermal limit the width of the resistance valley is used as an estimate.

The nature of weak link is determined by fitting the temperature dependence of the supercurrent. For diffusive SNS junctions, i.e. junctions where the junction length $L$ is larger than the electron mean free path, the Usadel equation is used to describe the temperature dependence [52-54],

$$
J=\frac{2 \pi k_{B} T}{e \rho_{N}} \operatorname{Im} \sum_{\omega_{n}>0} \frac{G_{N}^{2}}{\omega_{n}^{2}} \Phi_{N} \frac{d}{d x} \Phi_{N}
$$

where $\rho_{N}$ is the resistivity of the N layer, $\Phi_{N}$ is the self-consistently determinded induced order parameter function in the $\mathrm{N}$ layer with $G_{N}$ the corresponding normal Green function and $\omega_{n}=\pi k_{B} T(2 n+1)$ the Matsubara frequencies, where $n \geqslant 0$ is integer. As there is no analytical expression for arbitrary length and barrier transparency, the expression was solved numerically with three fitting parameters [55], $\gamma=\frac{\rho_{s} \xi_{s}}{\rho_{N} \xi_{N}}, \gamma_{B}=\frac{R_{B}}{\rho_{N} \xi_{N}}$ and $\xi_{N}$. Here, $\gamma$ is the ratio between the resistivities and coherence lengths in the superconducting leads and the topological insulator surface state. The resistivity of $\mathrm{Nb}$ is much smaller than that of $\mathrm{Bi}_{1.5} \mathrm{Sb}_{0.5} \mathrm{Te}_{1.7} \mathrm{Se}_{1.3}$ which gives $\gamma \ll 1 . \gamma_{\mathrm{B}}$ is proportional to the interface resistance per unit area $R_{B}$ between the 
(a)
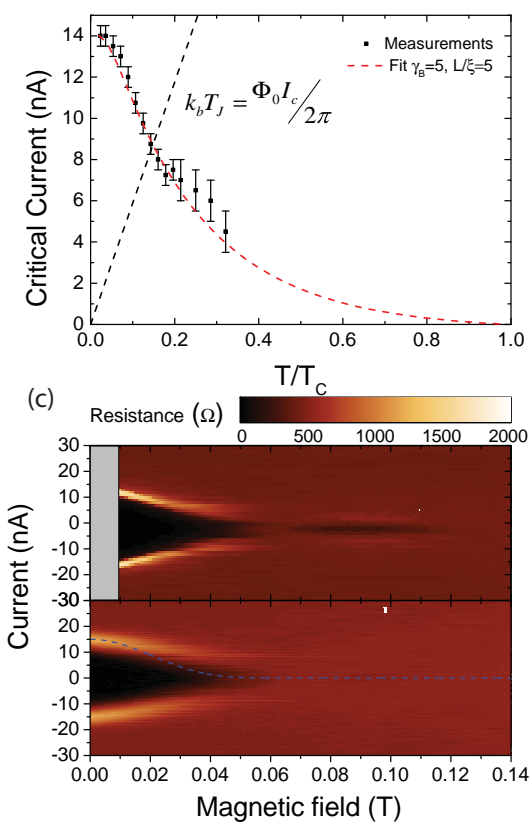

(b)

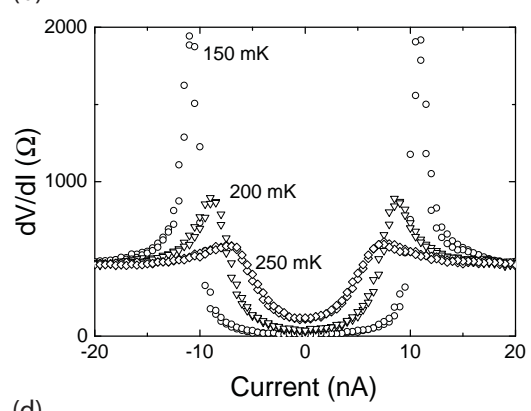

(d)

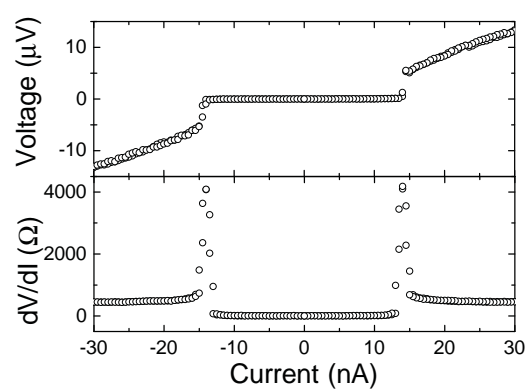

Figure 4.3: JOSEPHSON CHARACTERISTICS OF THE $\mathrm{NB} / \mathrm{BI}_{1.5} \mathrm{SB}_{0.5} \mathrm{Te}_{1.7^{-}}$ $\mathrm{SE}_{1.3} / \mathrm{Nb}$ JUnction illustrated in Figure 4.2. (a) Temperature dependence of the critical current. Above the thermal limit, indicated by the black dashed line, the critical current is determined by the resistance peaks. Below the thermal limit the width of the resistance dip is used as estimate. The red dashed line is a fit for diffusive junctions with the Usadel equation, see main text. (b) Derivative of the current-voltage characteristic above, at, and below the thermal limit. Below the thermal limit the resistance is zero and coherence peaks are visible. These peaks disappear above the thermal limit of $200 \mathrm{mK}$ and only a resistance valley remains. (c) Modulation of the critical current by an applied perpendicular magnetic field. The upper and lower frames are measurements during different cooldown cycles. The upper frame was the first cooldown using $\mathrm{Al}$ bond wires which have reduced cooling power below $10 \mathrm{mT}$, resulting in a sharp step in the critical current at this point. The bottom frame uses Au bond wires. This second cooldown exhibited a slightly reduced critical current and ergo reduced visibility of the first lobe of the diffraction pattern. The dotted line is a model of the critical current by Barzykin et al. [51] using the junction parameters found in (a). (d) Current-voltage characteristic and derivative at base temperature. The critical current of $14 \mathrm{nA}$ and normal state resistance of $460 \Omega$ result in an $\mathrm{I}_{\mathrm{C}} \mathrm{R}_{\mathrm{N}}$ product of $6.4 \mu \mathrm{V}$, consistent with a junction with high $\gamma_{B}$ and $L \geqslant \xi_{N}$. 
$S$ and $N$ layers. Finally, $\xi_{N}$ is the coherence length in the normal region at $T_{C}$. For the junction illustrated in figures 4.2(a) and 4.3 , the best fit is obtained for $\gamma_{B}=5$ and $\xi_{N}=11.4 \mathrm{~nm}$. The other junctions give values of the same order of magnitude. The large $\gamma_{\mathrm{B}}$ is in good agreement with the low excess current in the IV curves which implies a large barrier or low transparency at the interface. Taking the $T_{c}$ of the $\mathrm{Nb}$ wire, we get for $\xi_{N}\left(T_{c}\right)=\sqrt{\frac{\hbar D}{2 \pi k_{B} T_{c}}}$ a value of $28 \mathrm{~nm}$ which is also in good agreement with the fitting parameters. We note, that the junctions have a small $\mathrm{I}_{\mathrm{C}} \mathrm{R}_{\mathrm{N}}$ product, $\sim 7 \mu \mathrm{V}$. We argued that $\gamma \ll 1$. Together with the fitted values of $\gamma_{B}$ and $\xi_{N}$ the $I_{C} R_{N}$ product is either of the form $\propto V_{0} / \gamma_{B}$ or $I_{C} R_{N} \propto V_{0} e^{-L / \xi_{N}}$ [56]. Substitution of $\gamma_{B}, \xi_{N}$ and the junction length $L$ we estimate from this relation that the $I_{C} R_{N}$ should be in the order of $1-10 \mu V$ which is in good agreement with the measured product value.

\subsubsection{Critical current as a function of magnetic field}

In Fig. 4.3(c) measurements during two separate cooldowns of the $\mathrm{I}_{\mathrm{C}}(\mathrm{B})$ pattern at $30 \mathrm{mK}$ are shown for the same junction. The changes in the supercurrent in the second cooldown indicate a slight evolution in time. The upper frame shows the Fraunhofer pattern of the first measurement. Due to the use of $\mathrm{Al}$ bond wires, the cooling of the sample is reduced below the critical field of Al. These measurements are greyed out because the temperature increased in that region, we estimate by 100 $\mathrm{mK}$. A second cooldown using Au bonds results in the lower panel Fraunhofer pattern. Here, the critical current has reduced slightly resulting in a decreased visibility of the lobes of the Fraunhofer pattern. The Fraunhofer patterns are fitted by the critical current derived from the Usadel equations for arbitrary 
W and $\mathrm{L}$ with open boundaries, as described by Barzykin et al. [51],

$$
\begin{aligned}
I_{c} \sim & \sum_{l=-\infty}^{\infty}(-1)^{l} S_{l}(L / 2) S_{l}^{\prime}(L / 2) \times \\
& \left(\frac{\sin \pi(v+l) / 2}{\pi(v+l) / 2)}-(-1)^{l} \frac{\sin \pi(v-l) / 2}{\pi(v-l) / 2)}\right)^{2}, \\
S_{l}(u)= & \sqrt{|u| / 2 \pi}\left(q_{T}^{2}+\pi^{2} l^{2} / W^{2}\right)^{1 / 4} \times \\
& K_{1 / 2}\left(\sqrt{u^{2}\left(q_{T}^{2}+\pi^{2} l^{2} / W^{2}\right)}\right), \\
S_{l}^{\prime}(u)= & \frac{d}{d u} S_{l}(u),
\end{aligned}
$$

where $v=\phi / \phi_{0}$ with $\phi_{0}=\hbar / 2 e$ is the normalised flux, $\mathrm{q}_{\mathrm{T}}=$ $1 / \xi_{N}^{2}$ and $K_{1 / 2}$ is a modified Bessel function of the second kind. For our junction dimensions it follows from this model that the first period is tripled, i.e. the first minimum is at $\Phi=3 \Phi_{0}$ and the following minima are separated by $2 \Phi_{0}$ intervals. The doubling of the period is not restricted to diffusive junctions and was, in fact, also observed [57] and explained for [51] in ballistic junctions for width and length ratios in the order of one. The first minimum in the measurements occurs at a field value of $0.07 \mathrm{~T}$. The width of the junction is $37 \mathrm{~nm}$. For a flux of $3 \phi_{0}=6.2 \cdot 10^{-15} \mathrm{~Wb}$ this implies that the junction effective length (including the penetration depths) is about $2 \mu \mathrm{m}$. The obtained London penetration depth is then about $1 \mu \mathrm{m}$. This large London penetration depth compared to bulk $\mathrm{Nb}$ (47 $\mathrm{nm}$ ) [58] can be explained by the reduced dimensions of the junctions. The increase of the London penetration depth with decreasing film thickness is studied in Ref. [59]. A film thickness of $25 \mathrm{~nm}$ gives a London penetration depth above $100 \mathrm{~nm}$ and a decrease of the critical current to $8 \mathrm{~K}$. Due to the reduction of width of the junction in our devices, we end up with a $T_{C}$ of $1.4 \mathrm{~K}$ which consistently implies an even larger increase of the London penetration depth. The coherence length found from the fitting of the critical current together with the width and the length of the junction and London penetration depth serve as inputs for the model described in equation 4.3. The theoretical expectation is shown in figure 4.3 (c). The relative small critical current of the higher order peaks in the data is found to be well explained by the model. 


\section{$4 \cdot 4$ DISCUSSION}

We showed that the transport in the $\mathrm{Bi}_{1.5} \mathrm{Sb}_{0.5} \mathrm{Te}_{1.7} \mathrm{Se}_{1.3}$ flakes is dominated by surface states at low temperatures where we study proximity induced superconductivity. We prepared Josephson junctions with widths in the order of $40 \mathrm{~nm}$ and lengths in the order of 50 to $80 \mathrm{~nm}$ on several $\mathrm{Bi}_{1.5} \mathrm{Sb}_{0.5} \mathrm{Te}_{1.7} \mathrm{Se}_{1.3}$ flakes and measured them during several cooldowns to $30 \mathrm{mK}$. The Fraunhofer patterns unequivocally reveal that the supercurrent is a Josephson supercurrent. The measured critical currents are reproducibly observed on different devices and upon multiple cooldowns, and the measurement can be well explained by diffusive transport models and geometric effects. The predicted $4 \pi$ periodic Josephson effect can only be observed in the future for similar devices with just a single perpendicular mode [6o] and when measured faster than the relaxation rate of a quasiparticle inside an Andreev bound state.

The realisation of a Josephson supercurrent in junctions with dimensions in the order of tens of nanometers on a topological insulator dominated by surface states at low temperatures, is an important technological step towards advanced devices necessary for the observation of a quantised supercurrent and confirming the presence of a Majorana bound state in such devices [20]. Future work will focus on top and bottom gating of the surface states to eliminate potential additional trivial surface states from band bending.

BIBLIOGRAPHY

[1] L. Fu, C. L. Kane, and E. J. Mele, Phys. Rev. Lett. 98, 106803 (2007).

[2] L. Fu and C. L. Kane, Phys. Rev. B 76, 045302 (2007).

[3] D. Hsieh, D. Qian, L. Wray, Y. Xia, Y. S. Hor, R. J. Cava, and M. Z. Hasan, Nature 452, 970 (2008).

[4] Y. Xia, D. Qian, D. Hsieh, L. Wray, A. Pal, H. Lin, A. Bansil, D. Grauer, Y. S. Hor, R. J. Cava, and M. Z. Hasan, Nat Phys 5, 398 (2009).

[5] D. Hsieh, Y. Xia, L. Wray, D. Qian, A. Pal, J. H. Dil, J. Osterwalder, F. Meier, G. Bihlmayer, C. L. Kane, Y. S. Hor, et al., Science 323, 919 (2009). 
[6] H. Zhang, C.-X. Liu, X.-L. Qi, X. Dai, Z. Fang, and S.-C. Zhang, Nat Phys 5, 438 (2009).

[7] M. Z. Hasan and C. L. Kane, Rev. Mod. Phys. 82, 3045 (2010).

[8] C. Brüne, A. Roth, H. Buhmann, E. M. Hankiewicz, L. W. Molenkamp, J. Maciejko, X.-L. Qi, and S.-C. Zhang, Nat Phys 8, 485 (2012).

[9] G. Tkachov and E. M. Hankiewicz, physica status solidi (b) 250 (2013).

[10] D. Hsieh, Y. Xia, D. Qian, L. Wray, J. H. Dil, F. Meier, J. Osterwalder, L. Patthey, J. G. Checkelsky, N. P. Ong, A. V. Fedorov, et al., Nature 460, 1101 (2009).

[11] C. Jozwiak, C.-H. Park, K. Gotlieb, C. Hwang, D.-H. Lee, S. G. Louie, J. D. Denlinger, C. R. Rotundu, R. J. Birgeneau, Z. Hussain, and A. Lanzara, Nat Phys 9, 293 (2013).

[12] C. Li, O. van 't Erve, J. Robinson, Y. Liu, L. Li, and B. , Jonker, Nat Nano 9, 218 (2014).

[13] A. C. Potter and P. A. Lee, Phys. Rev. B 83, 184520 (2011).

[14] N. Read and D. Green, Phys. Rev. B 61, 10267 (2000).

[15] J. Nilsson, A. R. Akhmerov, and C. W. J. Beenakker, Phys. Rev. Lett. 101, 120403 (2008).

[16] Y. Tanaka, T. Yokoyama, and N. Nagaosa, Phys. Rev. Lett. 103, 107002 (2009).

[17] J. Alicea, Reports on Progress in Physics 75, 076501 (2012).

[18] C. Beenakker, Annual Review of Condensed Matter Physics 4, 113 (2013).

[19] S. Sasaki, M. Kriener, K. Segawa, K. Yada, Y. Tanaka, M. Sato, and Y. Ando, Phys. Rev. Lett. 107, 217001 (2011).

[20] L. Fu and C. L. Kane, Phys. Rev. Lett. 100, 096407 (2008).

[21] T. D. Stanescu, J. D. Sau, R. M. Lutchyn, and S. Das Sarma, Phys. Rev. B 81, 241310 (2010).

[22] D. Zhang, J. Wang, A. M. DaSilva, J. S. Lee, H. R. Gutierrez, M. H. W. Chan, J. Jain, and N. Samarth, Phys. Rev. B 84, 165120 (2011). 
[23] Z. Ren, A. A. Taskin, S. Sasaki, K. Segawa, and Y. Ando, Phys. Rev. B 82, 241306 (2010).

[24] Z. Ren, A. A. Taskin, S. Sasaki, K. Segawa, and Y. Ando, Phys. Rev. B 84, 165311 (2011).

[25] M. Veldhorst, M. Snelder, M. Hoek, T. Gang, V. K. Guduru, X. L. Wang, U. Zeitler, W. G. van der Wiel, A. A. Golubov, H. Hilgenkamp, and A. Brinkman, Nature Materials 11, 417 (2012).

[26] B. Sacépé, J. B. Oostinga, J. Li, A. Ubaldini, N. J. Couto, E. Giannini, and A. F. Morpurgo, Nat Communications 2, 575 (2011).

[27] F. Qu, F. Yang, J. Shen, Y. Ding, J. Chen, Z. Ji, G. Liu, J. Fan, X. Jing, C. Yang, and L. Lu, Sci. Rep. 2 (2012).

[28] J. R. Williams, A. J. Bestwick, P. Gallagher, S. S. Hong, Y. Cui, A. S. Bleich, J. G. Analytis, I. R. Fisher, and D. Goldhaber-Gordon, Phys. Rev. Lett. 109, 056803 (2012).

[29] V. Orlyanchik, M. P. Stehno, C. D. Nugroho, P. Ghaemi, M. Brahlek, N. Koirala, S. Oh, and D. J. Van Harlingen, ArXiv e-prints 1309.0163 (2013).

[30] S. Cho, B. Dellabetta, A. Yang, J. Schneeloch, Z. Xu, T. Valla, G. Gu, M. J. Gilbert, and N. Mason, Nat Commun 4, 1689 (2013).

[31] M.-X. Wang, C. Liu, J.-P. Xu, F. Yang, L. Miao, M.-Y. Yao, C. L. Gao, C. Shen, X. Ma, X. Chen, Z.-A. Xu, et al., Science 336,52 (2012).

[32] J. B. Oostinga, L. Maier, P. Schüffelgen, D. Knott, C. Ames, C. Brüne, G. Tkachov, H. Buhmann, and L. W. Molenkamp, Phys. Rev. X 3, 021007 (2013).

[33] L. Maier, J. B. Oostinga, D. Knott, C. Brüne, P. Virtanen, G. Tkachov, E. M. Hankiewicz, C. Gould, H. Buhmann, and L. W. Molenkamp, Phys. Rev. Lett. 109, 186806 (2012).

[34] L. Galletti, S. Charpentier, M. Iavarone, P. Lucignano, D. Massarotti, R. Arpaia, Y. Suzuki, K. Kadowaki, T. Bauch, A. Tagliacozzo, F. Tafuri, et al., Phys. Rev. B 89, 134512 (2014). 
[35] I. Sochnikov, A. J. Bestwick, J. R. Williams, T. M. Lippman, I. R. Fisher, D. Goldhaber-Gordon, J. R. Kirtley, and K. A. Moler, Nano Letters 13, 3086 (2013).

[36] M. Veldhorst, C. G. Molenaar, X. L. Wang, H. Hilgenkamp, and A. Brinkman, Applied Physics Letters 100, 072602 (2012).

[37] C. Kurter, A. D. K. Finck, P. Ghaemi, Y. S. Hor, and D. J. Van Harlingen, Phys. Rev. B 90, 014501 (2014).

[38] M. Veldhorst, M. Snelder, M. Hoek, C. G. Molenaar, D. P. Leusink, A. A. Golubov, H. Hilgenkamp, and A. Brinkman, physica status solidi (RRL) 7, 26 (2013).

[39] A. A. Taskin, Z. Ren, S. Sasaki, K. Segawa, and Y. Ando, Phys. Rev. Lett. 107, 016801 (2011).

[40] M. König, S. Wiedmann, C. Brüne, A. Roth, H. Buhmann, L. W. Molenkamp, X.-L. Qi, and S.-C. Zhang, Science 318, 766 (2007).

[41] A. Roth, C. Brüne, H. Buhmann, L. W. Molenkamp, J. Maciejko, X.-L. Qi, and S.-C. Zhang, Science 325, 294 (2009).

[42] C. Bouvier, T. Meunier, P. Ballet, X. Baudry, R. Bernd Günter Kramer, and L. Lévy, ArXiv e-prints 1112.2092 (2011).

[43] C. W. J. Beenakker, J. P. Dahlhaus, M. Wimmer, and A. R. Akhmerov, Phys. Rev. B 83, o85413 (2011).

[44] C. W. J. Beenakker and H. van Houten, Phys. Rev. Lett. 66, 3056 (1991).

[45] A. Furusaki, H. Takayanagi, and M. Tsukada, Phys. Rev. B 45, 10563 (1992).

[46] Y. Pan, et al. In preparation (2014).

[47] S. Hikami, A. I. Larkin, and Y. Nagaoka, Progress of Theoretical Physics 63, 707 (1980).

[48] M. S. Golden, Private communication (2014).

[49] R. Ferrell, Zeitschrift für Physik 182, I (1964).

[50] F. Mueller, R. N. Schouten, M. Brauns, T. Gang, W. H. Lim, N. S. Lai, A. S. Dzurak, W. G. van der Wiel, and F. A. Zwanenburg, Review of Scientific Instruments 84, 044706 (2013). 
[51] V. Barzykin and A. M. Zagoskin, Superlattices and Microstructures 25, 797 (1999).

[52] K. D. Usadel, Phys. Rev. Lett. 25, 507 (1970).

[53] A. Zaitsev, Sov. Phys. JETP 59, 1015 (1984).

[54] M. Y. Kupriyanov and V. Lukichev, Sov. Phys. JETP 67, 1163 (1988).

[55] A. A. Golubov, E. P. Houwman, J. G. Gijsbertsen, V. M. Krasnov, J. Flokstra, H. Rogalla, and M. Y. Kupriyanov, Phys. Rev. B 51, 1073 (1995).

[56] A. A. Golubov, M. Y. Kupriyanov, and E. Il'ichev, Rev. Mod. Phys. 76, 411 (2004).

[57] J. P. Heida, B. J. van Wees, J. J. Kuipers, T. M. Klapwijk, and G. Borghs, Phys. Rev. B 57, 11911 (1998).

[58] B. W. Maxfield and W. L. McLean, Phys. Rev. 139, A1515 (1965).

[59] A. I. Gubin, K. S. Il'in, S. A. Vitusevich, M. Siegel, and N. Klein, Phys. Rev. B 72, 064503 (2005).

[6o] M. Snelder, M. Veldhorst, A. A. Golubov, and A. Brinkman, Phys. Rev. B 87, 104507 (2013). 

We demonstrate topological insulator dc SQUIDs, based on superconducting $\mathrm{Nb}$ leads coupled to nanofabricated $\mathrm{Nb}-\mathrm{Bi}_{2} \mathrm{Te}_{3}-\mathrm{Nb}$ Josephson junctions. The high reproducibility of the fabrication process allows the creation of dc SQUIDs, which are in agreement with the design values. Clear critical current modulation of both the junctions and the SQUID with applied magnetic fields have been observed. We show that the SQUIDs have $\phi_{0}$ periodicity, of relevance to the Majorana fermion predicted to be a bound state in superconductor - topological insulator structures.

\subsection{INTRODUCTION}

Topological insulators [1-12] are characterised by an insulating bulk with a finite band gap and conducting edge or surface states, where charge carriers are protected against backscattering. These states give rise to the quantum spin Hall effect [2] without an external magnetic field, where electrons with opposite spins have opposite momentum at a given edge. The surface energy spectrum of a three-dimensional topological insulator $[3,4]$ is formed by an odd number of Dirac cones with the spin locked to the momentum. The long-sought yet elusive Majorana fermion [13] is predicted to appear in a combination of a superconductor and a topological insulator [14-16].

In the search for the Majorana fermion, efforts have been made to contact a topological insulator (TI) to a superconductor $(S)$ and supercurrents in S-TI-S junctions have been reported [17-19]. Evidences for supercurrents through the topological surface states are the demonstration of ballistic junctions over

This chapter is published by M. Veldhorst, C. G. Molenaar, X. L. Wang, H. Hilgenkamp \& A. Brinkman, Appl. Phys. Lett. 100072602 (2012).

The author of this PhD thesis has contributed to this publication by the measurements presented in figures 5.3-5.6, the analysis and interpretation of the data and by contributing in the writing of the manuscript. 


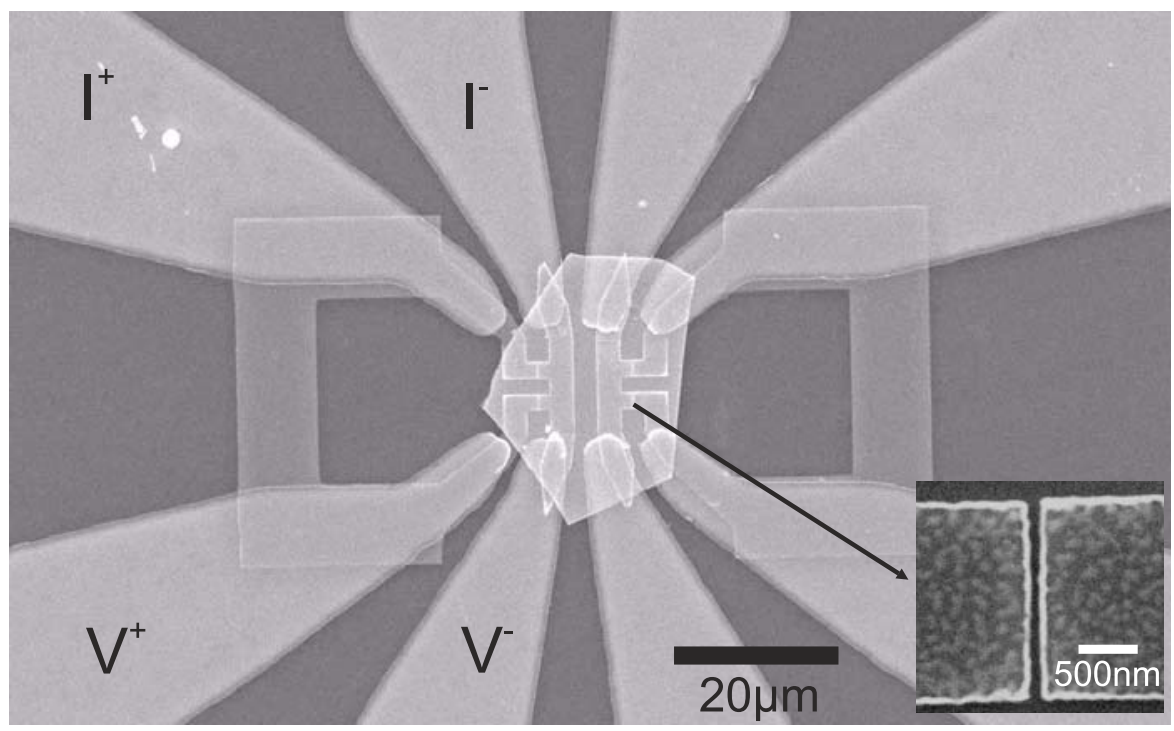

Figure 5.1: Topological INSUlator NANO DC SQUIDs. Two SQUIDs with junction seperation $1=120 \mathrm{~nm}$ are designed on an exfoliated $\mathrm{Bi}_{2} \mathrm{Te}_{3}$ (200 $\mathrm{nm}$ height) flake. The two $\mathrm{Nb}$ arms are differently sized resulting in an asymmetric SQUID. $\mathrm{Nb}$ is sputter deposited and defined by e-beam lithography.

length scales that are only consistent with the surface states [19]. Josephson effects have also been demonstrated [19].

In this chapter, we take a step towards the observation of the Majorana fermion with the fabrication of topological insulator dc SQUIDs. Majorana bound states may appear in the vortex of a topological superconductor and at the S-TI interface $[14,16]$. In the latter, time reversal symmetry in the topological insulator needs to be broken, for example by a magnetic insulator or an external magnetic field. Intriguing systems are proposed to identify the appearance of this exotic particle [14$16,20]$. Since the Majorana fermion is charge neutral and is a zero energy state, most proposals rely upon quantum interference devices. For example, a current-phase relationship with $4 \pi$ periodicity [14] might result from the interplay between the Majorana fermion and a superconductor. This periodicity might be detected in equilibrium states or in noise measurements [21]. Here, we present the fabrication of topological insulator SQUIDs and study their current-phase relationship. Furthermore, the interplay between superconductivity and strong spin orbit coupling in topological insulators might result in exotic phenomena and influence the current-phase relationship. 


\subsection{DEVICE FABRICATION*}

Figure 5.1 shows two superconductor $\mathrm{Nb}$ - topological insulator $\mathrm{Bi}_{2} \mathrm{Te}_{3}$ dc SQUIDs fabricated on one $\mathrm{Bi}_{2} \mathrm{Te}_{3}$ flake. Using mechanical exfoliation, $\mathrm{Bi}_{2} \mathrm{Te}_{3}$ flakes ${ }^{\dagger}$ ranging from $30 \mathrm{~nm}$ to $1 \mu \mathrm{m}$ are transferred to a $\mathrm{Si}$ substrate. $\mathrm{The}^{\mathrm{Bi}} \mathrm{Te}_{3}$ flakes are nanometer smooth on the $\mu \mathrm{m}$ scale. Figure 5.2 shows an AFM-image of the surface of a typical $\mathrm{Bi}_{2} \mathrm{Te}_{3}$ flake, revealing the 1 .o nm quintuple unit cell layers of $\mathrm{Bi}_{2} \mathrm{Te}_{3}$. After exfoliation, a superconducting $\left(\mathrm{T}_{\mathrm{C}}=\right.$ SIgK $) \mathrm{Nb}$-layer $(200 \mathrm{~nm})$ is sputter deposited with a $5 \mathrm{~nm}$ Pd layer deposited in situ on top to protect the $\mathrm{Nb}$ against oxidation. Electrodes are defined by photolithography. The $\mathrm{Nb}$ on top of the $\mathrm{Bi}_{2} \mathrm{Te}_{3}$ flake makes a strong superconducting contact with the $\mathrm{Nb}$ on the substrate. Finally, nanojunctions are defined by lift-off e-beam lithography and sputter deposition of $\mathrm{Nb}$. The substrate is slightly conducting $(\rho=5 \Omega \mathrm{cm})$ at room temperature to increase the resolution of e-beam lithography, but is completely insulating at low temperatures. Prior to deposition, in situ Ar-ion etching is performed in order to make transparent contacts. It is found that Ar etching roughens the $\mathrm{Bi}_{2} \mathrm{Te}_{3}$ surface, probably by preferential etching, but 2 minute etching at $50 \mathrm{eV}$ leaves a $\mathrm{Bi}_{2} \mathrm{Te}_{3}$ surface with $1-2 \mathrm{~nm}$ roughness and a transparent contact. Throughout the fabrication process the $\mathrm{Bi}_{2} \mathrm{Te}_{3}$ surface in between the $\mathrm{Nb}$ leads has only been covered by resist, leaving the surface of the junction barrier layer unaffected from Ar-ion etching and deposition steps. Two separate electrodes connected by $\mathrm{Nb}$ over the $\mathrm{Bi}_{2} \mathrm{Te}_{3}$ flake, for example the positive current and voltage leads, have a superconducting contact with a critical current exceeding $30 \mathrm{~mA}$, ensuring a large supercurrent through all layers.

\section{$5 \cdot 3$ CHARACTERISATION}

We have designed two SQUIDs on one $\mathrm{Bi}_{2} \mathrm{Te}_{3}$ flake (200 nm height), see figure 5.1. The nanojunctions are $2 \mu \mathrm{m}$ in width and $120 \mathrm{~nm}$ in length. The inductance ratio between the two arms is about $\lambda=0.2$. In a square washer approximation [22] the total enclosed area of the SQUID is $920 \mu \mathrm{m}^{2}$, and the estimated inductance $46 \mathrm{pH}$. Both SQUIDs showed similar behaviour, but in the rest of the paper we focus on the left SQUID.

${ }^{*}$ Device fabrication by M. Veldhorst.

${ }^{\dagger}$ Crystals grown by X. L. Wang. 


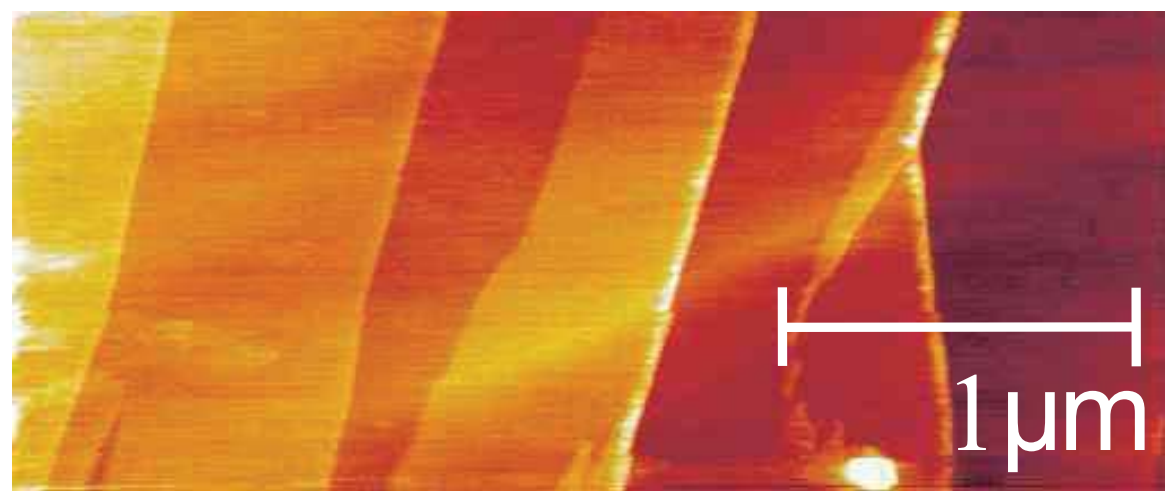

Figure 5.2: AFM-IMAGE of A BI $2 \mathrm{Te}_{3}$ FLAKE. Atomic force microscopy image of an exfoliated $\mathrm{Bi}_{2} \mathrm{Te}_{3}$ surface. The step edges are 1.0 $\mathrm{nm}$ high, corresponding to the $\mathrm{Bi}_{2} \mathrm{Te}_{3}$ quintuple unit cell. These nanometer flat surfaces span an area up to 50 $\times 50 \mu \mathrm{m}^{2}$.

Below $6 \mathrm{~K}$, the superconducting proximity effect induces a Josephson supercurrent through the junctions and at $1.4 \mathrm{~K}$ the dc SQUID has a critical current of $30 \mu \mathrm{A}$, as shown in figure 5.3. This results in critical current density of $7.5 \mathrm{~A} / \mathrm{m}$, which is within $10 \%$ of individually measured junctions on different flakes. The SQUID had a critical current constant within 10\% over three cooldowns running over two weeks. Due to shunting of the junctions we find a relatively low $I_{C} R_{N}=10 \mu \mathrm{V}$. One of the major reasons for this low $I_{C} R_{N}$ product is the large bulk shunt reducing the normal state resistance [19]. Ballistic junctions with $\xi_{0} \approx 80 \mathrm{~nm}, \mathrm{~T}_{\mathrm{C}}=6 \mathrm{~K}$ and $1=120 \mathrm{~nm}$, have an estimated $\mathrm{I}_{C} \mathrm{R}_{\mathrm{N}} \approx 130-260 \mu \mathrm{V}$, but the bulk conductance reduces the characteristic voltage to a few percent. In order to obtain higher values, electrical gating or chemical substitution, e.g. $\mathrm{Bi}_{2} \mathrm{Se}_{3} \mathrm{Te}_{3}$ [23], could be used. Nonetheless, clear supercurrents are observed.

Applying an external magnetic field causes oscillations of the superconducting critical currents due to interference of the two arms, shown in 5.4. This unequivocally demonstrates the Nb$\mathrm{Bi}_{2} \mathrm{Te}_{3}$ SQUID. Since these junctions are in the ballistic limit [19], we rule out superconducting transport through the diffusive bulk, and conclude that the supercurrent is carried by the topological surface states, which are present as demonstrated in high magnetic field measurements [19]. This allows us to study the current-phase relationship of superconductor - topological surface state structures. 


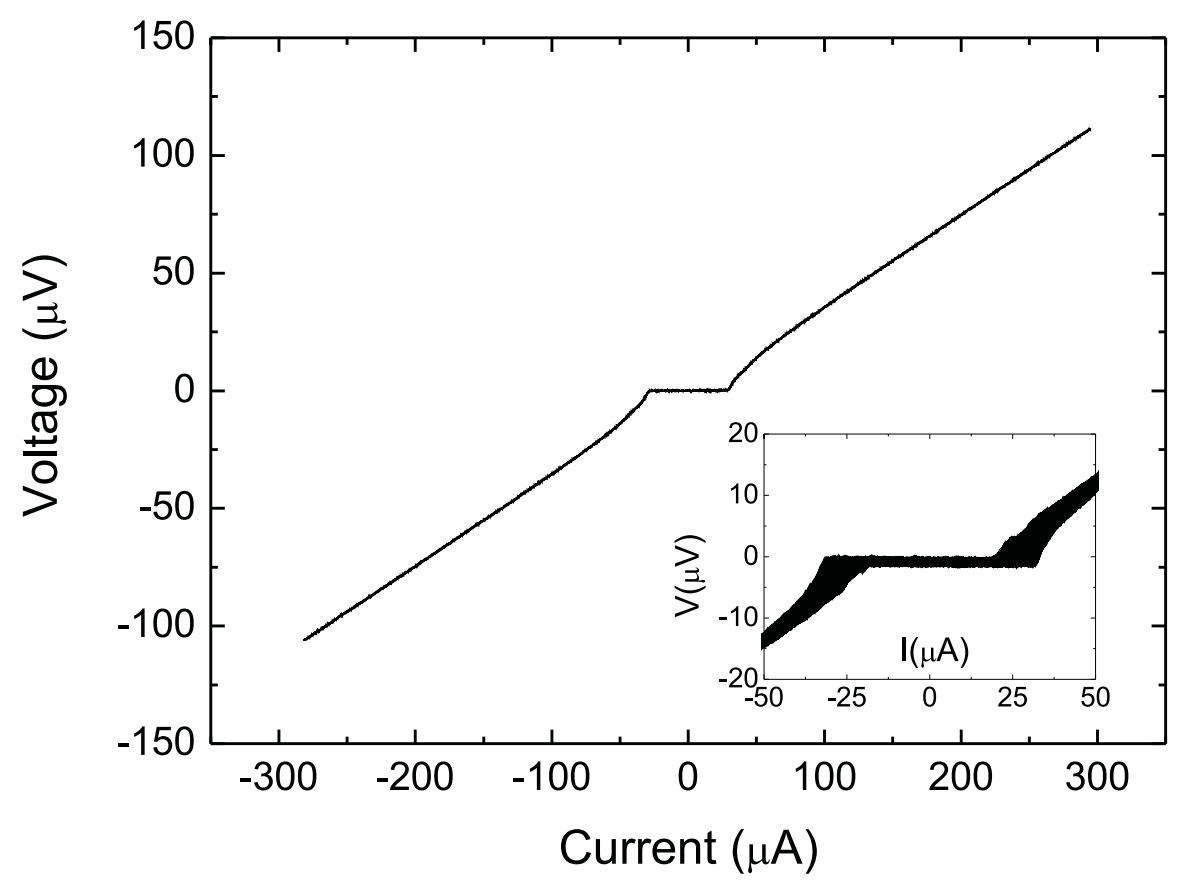

Figure 5.3: IV-CURVE OF THE DC SQUID. Typical SQUID critical current of $30 \mu \mathrm{A}$. The junctions are $\mu \mathrm{m}$ in width and have an electrode separation of $120 \mathrm{~nm}$. The intrinsic bulk shunt has reduced the $I_{C} R_{N}$ product to $10 \mu V$.

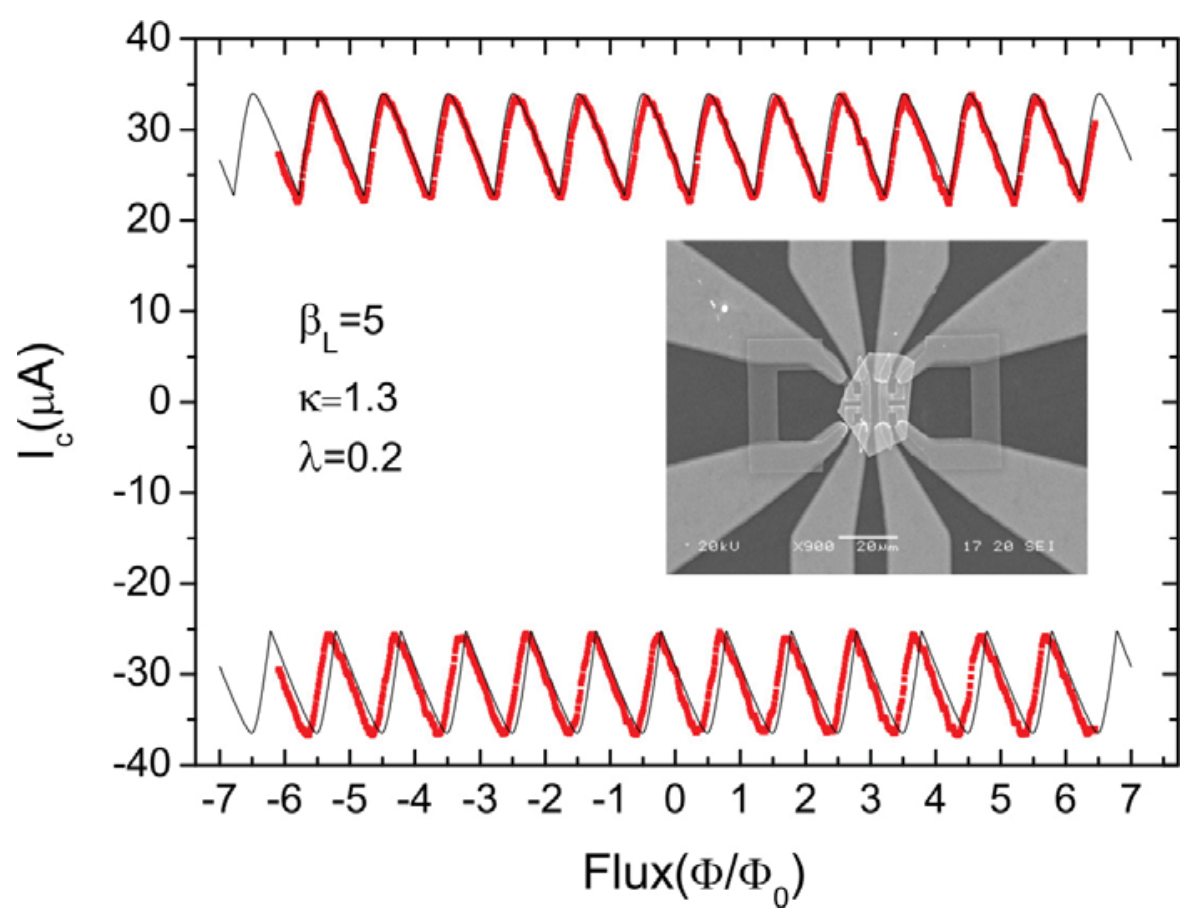

Figure 5.4: CRITICAL CURRENT OF THE DC SQUID. The critical current of the SQUID varies between 22 and $34 \mu \mathrm{A}$. The measurements, in red, are fitted with a model accounting for asymmetry in the arms, $\lambda$, and asymmetry in the junctions, $\mathrm{k}$. 


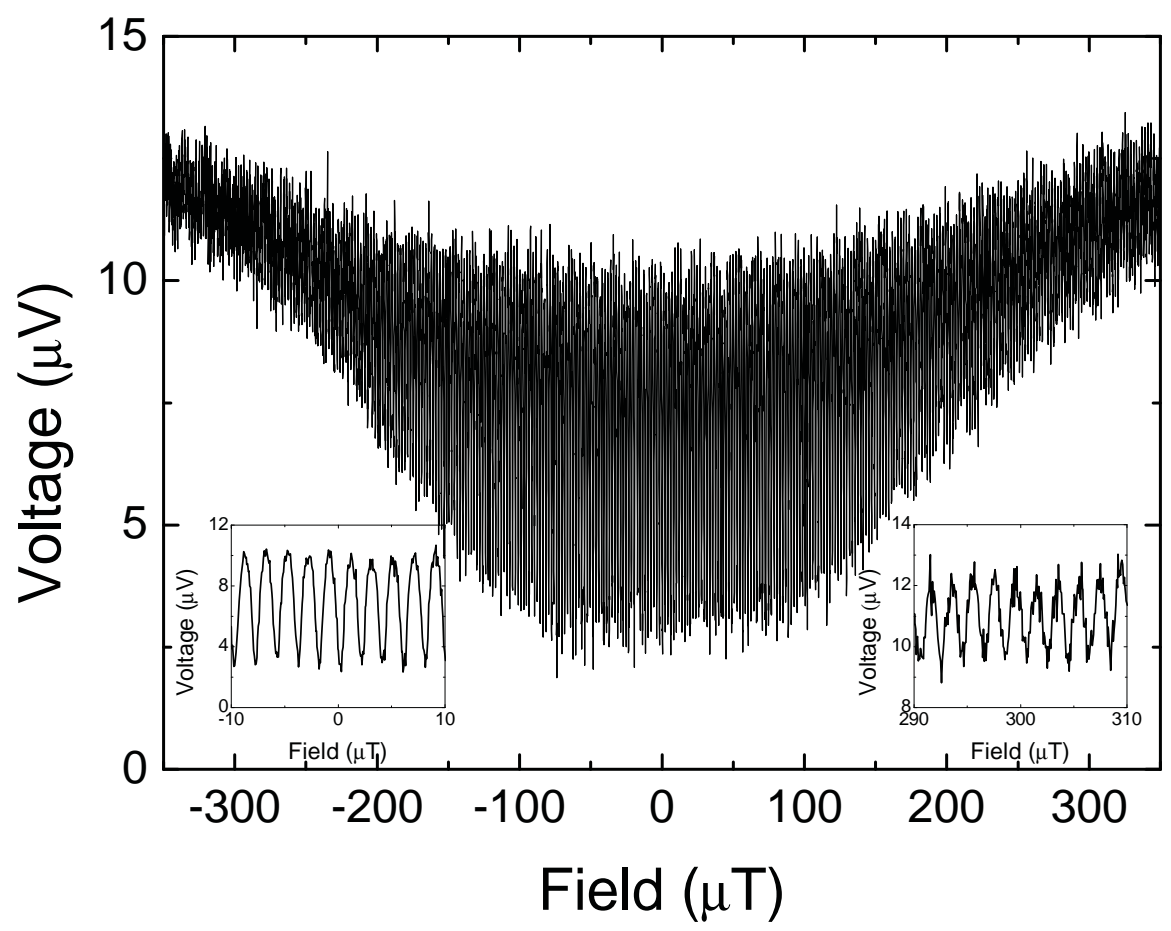

Figure 5.5: VolTAGE MODULATION OF THE DC SQUID AND INDIVIDUAL JUNCTIONS. The $\mathrm{V}-\Phi$ modulation of the SQUID is measured at a bias current of $34 \mu \mathrm{A}$. The SQUID oscillations are periodic in $2 \mu \mathrm{T}$, while the junction critical current is minimal at $350 \mu \mathrm{T}$, corresponding to the first minimum of the Fraunhofer pattern. SQUID oscillations are still observable. 


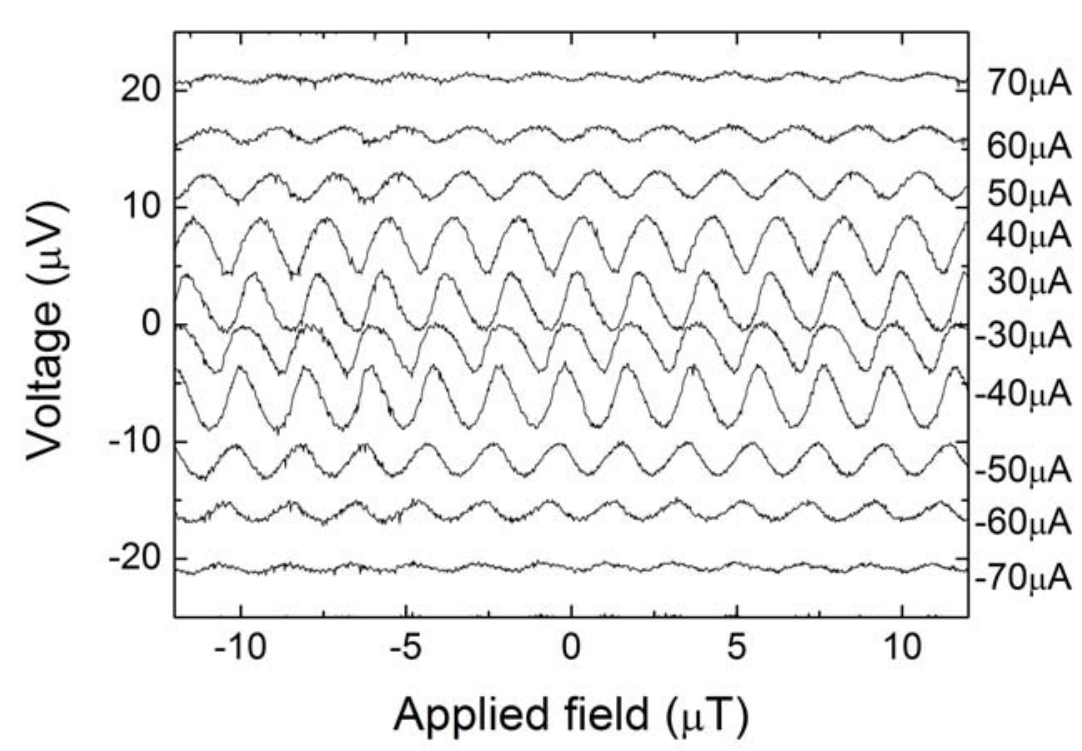

Figure 5.6: VolTAGE MOdULATION OF THE DC SQUID. The V- $\phi$ relationship reveals an asymmetric response as well as a shift for increasing bias currents. The solid lines represent equicurrents for $\pm 30-90 \mu \mathrm{A}$. The asymmetric response and shift is due to the asymmetric design of the SQUID arms.

The voltage modulation is strongest at a bias of approximately $40 \mu \mathrm{A}$ (figure 5.6), which corresponds to a current close to the critical current of the SQUID. The critical current modulation is $\sim 35 \%$ of the total critical current. Using a simple model based on $I_{C} \sin \phi, \beta_{L}$ together with the relative inductance, we can fit the field dependence with high accuracy. The asymmetrical bias causes asymmetry in the current phase relationship, Fig. 5.4. We also observe a shift in the current-phase relationship when increasing the bias current. The two arms of the SQUID have different inductances, which results in a growing field threading the SQUID for increasing current. The ${ }_{3} / 4 \Phi_{0}$ shift in the $\mathrm{V}-\phi$ relation at $90 \mu \mathrm{A}$ bias can be accounted for by a $10 \mathrm{pH}$ inductance difference between the two arms. This corresponds to the fitted values for the total inductance of the SQUID, $L=\frac{\Phi_{0}}{2 \pi} \frac{\beta_{L}}{I_{c}}=55 \mathrm{pH}$ and the asymmetry factor $\lambda=0.2$, resulting in an inductance difference of $11 \mathrm{pH}$. At the optimum bias, the sensitivity of the SQUID is $15 \mu \mathrm{V} / \Phi_{0}$. In the limit $\beta_{\mathrm{L}}(=5)>>1$, the sensitivity of a SQUID can be estimated by $\mathrm{R} / \mathrm{L}[24]$ which yields $12 \mu \mathrm{V} / \Phi_{0}$. 


\section{$5 \cdot 4$ ANALYSIS}

As expected, the SQUID modulation frequency is much larger than that of the individual junctions, see Fig. 5.5. A SQUID oscillation corresponds to $1.9 \mu \mathrm{T}$, while the critical current of the junctions is suppressed at $350 \mu \mathrm{T}$. This corresponds to about 180 SQUID oscillations in a junction oscillation, which is slightly lower than expected by comparing the enclosed areas. However, for the enclosed area of a junction, the Josephson penetration depth and flux focussing has to be included, also observed in [19]. Using the square washer approximation, given a total SQUID area of $920 \mu^{2}$, in combination with the calibrated field of the used coil, we calculate a $2 \pi$ periodic dependence within $7 \%$ accuracy, enough to exclude a $4 \pi$ periodicity. Future experiments will point towards noise measurements were a $4 \pi$ periodic dependence is predicted to exist in nonequilibrium [21] and on the incorporation of ferromagnetic insulators to break time reversal symmetry.

\section{$5 \cdot 5$ CONCLUSIONS}

In conclusion, we have demonstrated the first $\mathrm{Nb}-\mathrm{Bi}_{2} \mathrm{Te}_{3} \mathrm{SQUID}$. As shown previously, the supercurrent is carried by the topological surface states, thereby allowing the study of the currentphase relationship of the superconductor - topological insulator structures. From the $\mathrm{V}-\phi$ characteristics we deduced a $2 \pi$ current-phase relationship. The high reproducibility allows to study the noise properties of these devices in future experiments and to eventually include magnetic insulators in order to break time reversal symmetry to create Majorana bound states.

\section{BIBLIOGRAPHY}

[1] B. A. Bernevig, T. L. Hughes, and S.-C. Zhang, Science 314, 1757 (2006).

[2] M. König, S. Wiedmann, C. Brüne, A. Roth, H. Buhmann, L. W. Molenkamp, X.-L. Qi, and S.-C. Zhang, Science 318, 766 (2007).

[3] L. Fu, C. L. Kane, and E. J. Mele, Phys. Rev. Lett. 98, 106803 (2007). 
[4] H. Zhang, C.-X. Liu, X.-L. Qi, X. Dai, Z. Fang, and S.-C. Zhang, Nat Phys 5, 438 (2009).

[5] X.-L. Qi, R. Li, J. Zang, and S.-C. Zhang, Science 323, 1184 (2009).

[6] D. Hsieh, D. Qian, L. Wray, Y. Xia, Y. S. Hor, R. J. Cava, and M. Z. Hasan, Nature 452, 970 (2008).

[7] Y. L. Chen, J. G. Analytis, J.-H. Chu, Z. K. Liu, S.-K. Mo, X. L. Qi, H. J. Zhang, D. H. Lu, X. Dai, Z. Fang, S. C. Zhang, et al., Science 325, 178 (2009).

[8] D. Hsieh, Y. Xia, D. Qian, L. Wray, J. H. Dil, F. Meier, J. Osterwalder, L. Patthey, J. G. Checkelsky, N. P. Ong, A. V. Fedorov, et al., Nature 460, 1101 (2009).

[9] D. Hsieh, Y. Xia, L. Wray, D. Qian, A. Pal, J. H. Dil, J. Osterwalder, F. Meier, G. Bihlmayer, C. L. Kane, Y. S. Hor, et al., Science 323, 919 (2009).

[10] H. Peng, K. Lai, D. Kong, S. Meister, Y. Chen, X.-L. Qi, S.-C. Zhang, Z.-X. Shen, and Y. Cui, Nat Mater 9, 225 (2010).

[11] P. Cheng, C. Song, T. Zhang, Y. Zhang, Y. Wang, J.-F. Jia, J. Wang, Y. Wang, B.-F. Zhu, X. Chen, X. Ma, et al., Phys. Rev. Lett. 105, 076801 (2010).

[12] T. Zhang, P. Cheng, X. Chen, J.-F. Jia, X. Ma, K. He, L. Wang, H. Zhang, X. Dai, Z. Fang, X. Xie, et al., Phys. Rev. Lett. 103, 266803 (2009).

[13] E. Majorana, Il Nuovo Cimento 14, 171 (1937).

[14] L. Fu and C. L. Kane, Phys. Rev. Lett. 100, 096407 (2008).

[15] J. Nilsson, A. R. Akhmerov, and C. W. J. Beenakker, Phys. Rev. Lett. 101, 120403 (2008).

[16] Y. Tanaka, T. Yokoyama, and N. Nagaosa, Phys. Rev. Lett. 103, 107002 (2009).

[17] D. Zhang, J. Wang, A. M. DaSilva, J. S. Lee, H. R. Gutierrez, M. H. W. Chan, J. Jain, and N. Samarth, Phys. Rev. B 84, 165120 (2011).

[18] B. Sacépé, J. B. Oostinga, J. Li, A. Ubaldini, N. J. Couto, E. Giannini, and A. F. Morpurgo, Nat Communications 2, 575 (2011). 
[19] M. Veldhorst, M. Snelder, M. Hoek, T. Gang, V. K. Guduru, X. L. Wang, U. Zeitler, W. G. van der Wiel, A. A. Golubov, H. Hilgenkamp, and A. Brinkman, Nature Materials 11, 417 (2012).

[20] C. Beenakker, Annual Review of Condensed Matter Physics 4, 113 (2013).

[21] D. M. Badiane, M. Houzet, and J. S. Meyer, Phys. Rev. Lett. 107, 177002 (2011).

[22] M. Ketchen, W. Gallagher, A. Kleinsasser, S. Murphy, and J. Clem, SQUID 85-Superconducting Quantum Interference Devices and their Applications (Berlin: de Gruyter, 1985), p. 865.

[23] S. Jia, H. Ji, E. Climent-Pascual, M. K. Fuccillo, M. E. Charles, J. Xiong, N. P. Ong, and R. J. Cava, Phys. Rev. B 84, 235206 (2011).

[24] C. Tesche and J. Clarke, Journal of Low Temperature Physics 29, 301 (1977). 
We have modelled SQUIDs with topologically nontrivial superconducting junctions and performed an optimisation study on the Majorana fermion detection. We find that the SQUID parameters $\beta_{\mathrm{L}}$, and $\beta_{\mathrm{C}}$ can be used to increase the ratio of Majorana tunnelling to standard Cooper pair tunnelling by more than two orders of magnitude. Most importantly, we show that dc SQUIDs including topologically trivial components can still host strong signatures of the Majorana fermion. This paves the way towards the experimental verification of the theoretically predicted Majorana fermion.

\section{I INTRODUCTION}

Superconducting junctions with topologically non-trivial barriers are predicted to host Majorana bound states [1, 2]. Nontrivial states include the edge or surface of the recently discovered topological insulators [3-12] and semiconducting nanowires in the presence of Rashba spin orbit coupling and a Zeeman field $[13,14]$. Candidates with high potential for the detection and manipulation of the Majorana fermion [15] are superconducting quantum interference devices (SQUIDs) [16, 17]. The appearance of Majorana bound states in superconducting junctions enables tunnelling of quasiparticles with charge $e$ across the junction, which doubles the Josephson periodicity, $I_{c}=$ $\mathrm{I}_{0} \sin (\phi / 2)[1]$. The doubled periodicity is predicted to lead to the absence of odd integer Shapiro steps in individual junctions, and a SQUID modulation period of $2 \Phi_{0}$ instead of the

This chapter is published by M. Veldhorst, C. G. Molenaar, C. J. M. Verwijs, H. Hilgenkamp \& A. Brinkman, Phys. Rev. B 86, 024509 (2012).

The author of this $\mathrm{PhD}$ thesis has contributed to this publication by contributing to the model, the interpretation of the results and by contributing in the writing of the manuscript. 
usual $\Phi_{0}$ periodicity, with $\Phi_{0}=\frac{h}{2 e}$ the magnetic flux quantum in superconductivity [16-18].

Experimental efforts have been made to contact superconductors to topologically non-trivial states [19-21]. Josephson effects have been observed, and SQUIDs have been reported [22]. The first signatures of a Majorana fermion, characterised by a zero bias conductance peak, have been observed in superconductor - semiconducting nanowire junctions [23]. Nonetheless, so far only $\Phi_{0}$ periodic dependences have been observed. Relaxation to equilibrium states $[16,18]$, quantum phase slips [17], and the large bulk shunt present in contacts with topological insulators so far, may reduce the $2 \Phi_{0}$ periodicity.

A key question therefore is how to optimise the Majorana character. Here, we study extrinsic parameters that can be controlled to optimise the $\sin (\phi / 2)$ signal from the Majorana fermion in dc SQUIDs composed of junctions containing both $\sin (\phi)$ and $\sin (\phi / 2)$ components in different proportions. Our main observation is that the SQUID parameters $\beta_{\mathrm{L}}$ and $\beta_{\mathrm{C}}$ are important parameters altering the periodicity. Furthermore, a superconducting interferometer will have the periodicity of the component with the smallest periodicity. Nonetheless, even in dc SQUIDs with topologically trivial components the Majorana character strongly influences the dc SQUID characteristics. This study is also of relevance for dc SQUIDs composed of junctions with higher order periodicities, occurring in SNS and SFS systems [24].

\subsection{MODEL}

In the next session we introduce fluxoid quantisation in superconducting rings composed of topologically trivial and nontrivial parts. Then we will use the fluxoid quantisation conditions to determine the critical current in the SQUID under applied magnetic fields and derive the voltage state assuming that the junctions can be described with the resistively and capacitively shunted junction model.

\subsubsection{Fluxoid quantisation in topologically (non)-trivial rings}

The fluxoid quantisation in a superconducting loop $\Gamma$ leads to $\gamma_{p l} \Phi_{0}$ periodicity, with $\gamma_{p l}$ related to the charge carrier $q=\frac{2 e}{\gamma_{p l}}$ in the loop. In macroscopic systems, $\gamma_{\mathrm{pl}}=1$, but for mesoscopic 

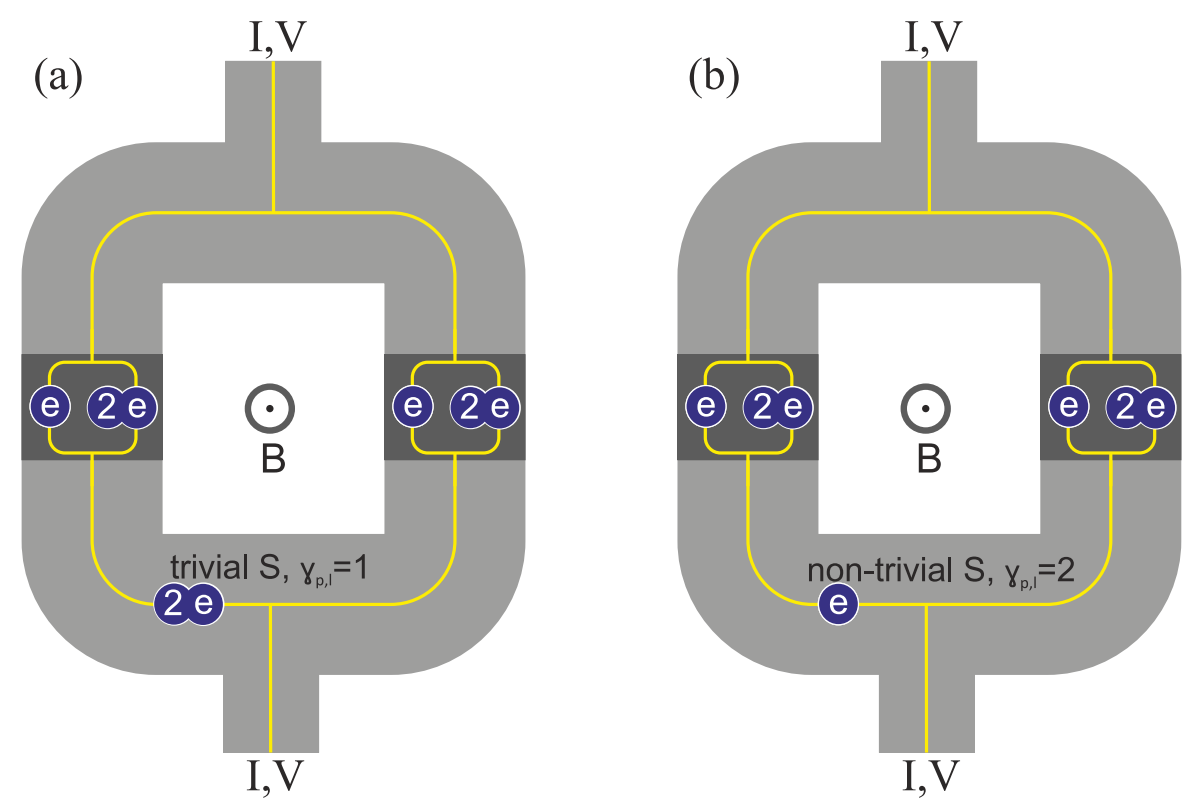

Junction: trivial $2 e \alpha \sin (\phi)$

non-trivial $\Theta(1-\alpha) \sin (\phi / 2)$

Figure 6.1: SCHEMATIC REPRESENTATION OF THE CONSIDERED DC SQUID. The dc SQUID is composed of a superconducting ring interrupted by two Josephson junctions. Charge transport through the Josephson junction is characterised by standard Cooper pair tunnelling $(\sin (\phi))$ and single electron tunnelling by virtue of the Majorana fermion $(\sin (\phi / 2))$. The relative contribution of these two processes is determined by the factor $\alpha$. We analyse two scenarios, in (a) the superconductor is macroscopic and is in the trivial state, $\gamma_{\mathrm{pl}}=1$ and in (b) the superconductor is in the topologically non-trivial state, $\gamma_{\mathrm{pl}}=2$. Doubled periodicity is only observed when there is a topologically non-trivial state in the entire ring, as in (b). However, the unusual current-phase relation changes the dc SQUID characteristics even when the ring is not entirely topologically nontrivial. 
systems on the order of the superconducting coherence length $\xi[25,26]$ and systems including Majorana fermions, $\gamma_{\mathrm{pl}}$ can be either 1 or 2 depending on parity conservation [27]. Integrating the phase of a superconducting loop containing $\mathrm{N}$ Josephson junctions results in:

$$
\oint \nabla \frac{\phi}{\gamma_{\mathrm{pl}}} \cdot \mathrm{d} \mathbf{l}=-\frac{2 \pi}{\gamma_{\mathrm{pl}} \Phi_{0}} \int_{\Gamma^{\prime}} \Lambda \mathbf{J}_{\mathrm{s}} \cdot \mathrm{d} \mathbf{l}-\frac{2 \pi}{\gamma_{\mathrm{pl}} \Phi_{0}} \oint \mathbf{A} \cdot \mathrm{d} \mathbf{l}-\sum_{i=1}^{\mathrm{N}} \frac{\phi_{\mathrm{i}}}{\gamma_{\mathrm{pj}}}
$$

Here, $\Gamma^{\prime}$ denotes the contour of the superconducting ring with the Josephson junctions excluded, and $\Lambda$ is a normalisation constant for the current. The phase-drop over junction $i$ is given by $\frac{\phi_{i}}{\gamma_{p j}}$, with $\gamma_{p j}$ connected to the charge carrier $q=\frac{2 e}{\gamma_{p j}}$ in the junction. We will consider scenarios where the superconductor is either trivial $\gamma_{\mathrm{pl}}=1$ or topologically non-trivial $\gamma_{\mathrm{pl}}=$ 2. Also we will consider the junctions to be trivial $\gamma_{p j}=1$, topologically non-trivial $\gamma_{p j}=2$ or that both charge carrier types are present in the junctions. When the junctions are topologically non-trivial, but the superconductor is macroscopic, quantum phase slips can occur in the superconductor so that $\gamma_{\mathrm{pl}}$ can be different from $\gamma_{p j}$. Contour integration over the magnetic vector potential A results in the total flux $\Phi$. Then, in the limit $\mathbf{J}_{s}=0$, assuming thick superconducting leads, fluxoid quantisation reduces to: $\frac{1}{2 \pi} \sum_{i=1}^{N} \frac{\phi_{i}}{\gamma_{p j}}+\frac{\Phi}{\gamma_{p l} \Phi_{0}}=n$. The flux $\Phi$ is the sum of the external flux and the self-flux induced by current flowing through the ring.

Now we will consider the case of a ring containing two junctions, as depicted in Fig. 6.1. We consider the dc SQUID to be symmetric, except for the current-phase relationship of the individual junctions. Inclusion of asymmetry (e.g. inductance, critical current or capacitance asymmetry) is easily included. However, inclusion will only lead to asymmetrical SQUID characteristics, and will not change the periodicity. The total flux of the considered system is given by $\Phi=\Phi_{e}+\mathrm{LI}_{c} \chi_{1}-\mathrm{LI}_{\mathrm{c}} \chi_{2}$. Here, $\Phi_{e}$ is the externally applied flux, L the inductance of a single arm and $I_{c}$ the critical current of the individual junctions 1 and $2, I_{c}=I_{c 1}=I_{c 2}$. The factors $\chi_{1,2}$ denote the current dependence on the phase difference of the individual junctions, which we limit to $\chi \chi_{0}^{-1}=\alpha \sin (\phi)+(1-\alpha) \sin (\phi / 2)$, with $\alpha \in[0,1]$ the relative amplitude and $\chi_{0}$ a normalisation factor to have $\max (\chi)=1$. The $\sin (\phi)$ component is the standard Josephson relation, and SNS-junctions are well described by this sinusoidal relation, but it can include higher order components 
due to $n$ Cooper pairs tunnelling [24], described by $\mathrm{I}_{s}(\phi)=$ $\sum_{n=1}^{\infty} I_{c}^{n} \sin \left(n \phi_{n}\right)$. Our simplification includes the lowest frequency, which is enough for our conclusions. The $\sin (\phi / 2) \mathrm{com}-$ ponent is due to single electron tunnelling by virtue of the Majorana fermion resulting in the $4 \pi$ current-phase relationship periodicity.

\subsubsection{SQUID characteristics in the superconducting and voltage state}

The critical current for an applied external field is obtained by finding the solution of the fluxoid quantisation equation with the maximal critical current. The junctions in the voltage state are modeled with the resistively and capacitively shunted junction (RCSJ) model, assuming an ideal Josephson junction shunted by a resistor $R$ and a capacitor $C: I=C \frac{d V}{d t}+I_{c} \chi_{1,2}+\frac{V}{R}$. The voltage is related to the time derivative of the phase by $V=\frac{\Phi_{0}}{2 \pi} \frac{d \phi}{d t}$, the same for a topologically trivial and non-trivial ring since we have written the phase as $\frac{\phi}{\gamma_{p j 1,2}}$. The RCSJ model leads to the expression $\frac{\mathrm{d}^{2} \phi}{\mathrm{dt}^{2}}+\frac{1}{\mathrm{RC}} \frac{\mathrm{d} \phi}{\mathrm{dt}}+w_{\mathrm{p}}^{2}\left(\chi_{1,2}-\frac{\mathrm{I}}{\mathrm{I}_{\mathrm{c}}}\right)=0$, with the plasma frequency $w_{p}=\sqrt{\frac{2 \pi}{\Phi_{0}} \frac{I_{c}}{C}}$. The SQUID parameters are defined as

$$
\begin{aligned}
& \beta_{\mathrm{L}}=\frac{2 \pi \mathrm{LI}_{\mathrm{c}}}{\Phi_{0}}, \\
& \beta_{\mathrm{C}}=\frac{2 \pi}{\Phi_{0}} \mathrm{I}_{\mathrm{C}} \mathrm{R}^{2} \mathrm{C} .
\end{aligned}
$$

Applying the fluxoid quantisation equation, the voltage state can be described by the two differential equations which we have solved numerically

$$
\beta_{C} \frac{d^{2} \phi_{2,1}}{d t^{2}}+\frac{d \phi_{2,1}}{d t}+\chi_{2,1}-\frac{1}{2} \frac{I}{I_{c}} \pm \beta_{L}^{-1}\left(\phi_{2}-\phi_{1}-2 \pi \frac{\Phi_{e}}{\Phi_{0}}\right)=0 .
$$

\subsection{RESULTS}

In this section we show the SQUID characteristics. We will start analysing a dc SQUID composed of a topologically trivial ring, and two non-trivial junctions. In this regime there is no doubled fluxoid quantisation, however the unusual current-phase 


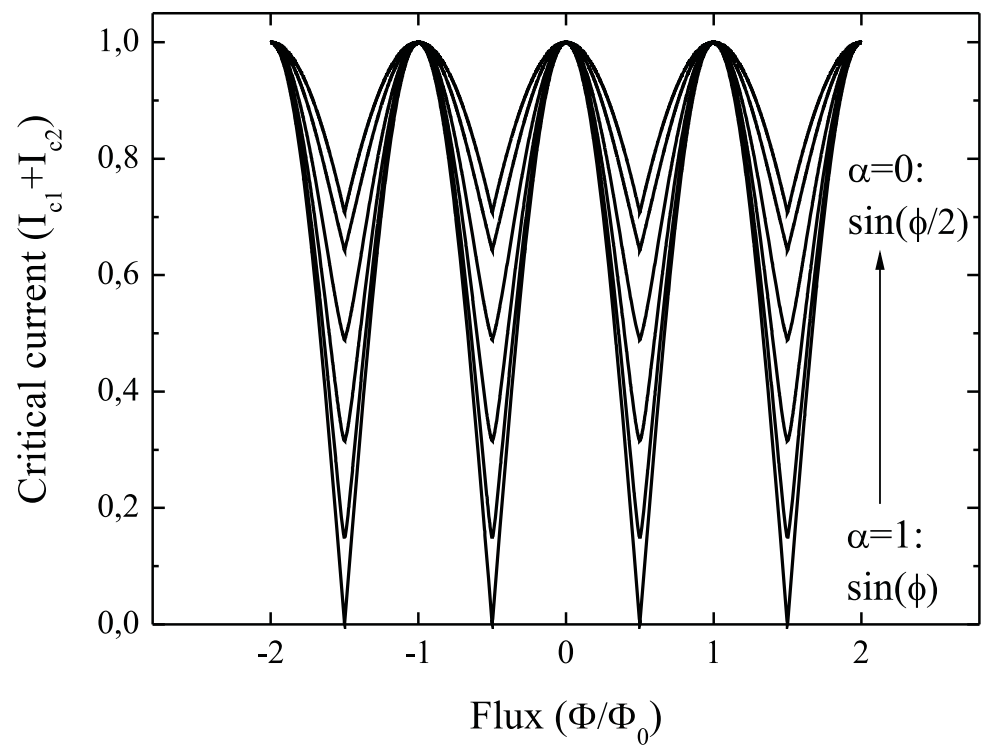

Figure 6.2: CURRENT-PHASE CHARACTERISTICS OF A DC SQUID COMPOSED OF A TOPOLOGICALLY TRIVIAL SUPERCONDUCTING RING AND NON-TRIVIAL JUNCTIONS. The current-phase relationship of the junctions is shown for $\alpha \sin (\phi)+(1-$ $\alpha) \sin (\phi / 2)$ in steps $\delta \alpha=0.2, \beta_{\mathrm{L}}=0$. The increase of the $\sin (\phi / 2)$ component causes a decrease of the oscillation amplitude without introducing a $2 \Phi_{0}$ component.

relationship causes a deviation from standard SQUID characteristics. After that, we will consider the SQUID in the entirely non-trivial regime, where doubled periodicity is observed due to the appearance of the Majorana fermion. Finally, we move to the voltage state and consider both cases in this regime.

\subsection{1 dc SQUIDs composed of trivial and non-trivial elements}

The considered dc SQUID is composed of a topologically trivial ring, and two non-trivial junctions, as shown in Fig. 6.1a. Figure 6.2 shows the critical current dependence of the dc SQUID. In this figure, the non-trivial junctions develop their current-phase relationship from pure $\sin (\phi)$ to pure $\sin (\phi / 2)$ in steps $\delta \alpha=0.2$. Note that all graphs are calculated for $\beta_{\mathrm{L}}=0$, a situation which for standard SQUIDs leads to a complete critical current modulation. The $2 \Phi_{0}$ periodicity due to the $\sin (\phi / 2)$ component, 
tends to be completely obscured by the trivial superconducting ring. Quantum phase slips cause the usual $\Phi_{0}$ periodicity, equivalent to what is calculated by Heck et al. [28] when one of the junctions is topologically trivial. When $\chi_{1}=\sin (\phi / 2)$ and $\chi_{2}=\sin (\phi)$ we obtain the result of Fig. 6.2 for $\alpha$ approximately 0.7 , shifted by an additional $\frac{1}{4} \Phi_{0}$. Instead of $2 \Phi_{0}$ periodicity, the $\sin (\phi / 2)$ component influences the modulation depth of the SQUID. There is no appearance of asymmetry as is the case for asymmetric SQUIDs, with different critical currents of the individual junctions [29]. The decrease in modulation depth by increasing the $\sin (\phi / 2)$ component looks similar to increasing $\beta_{\mathrm{L}}$ in standard SQUIDs. However, this is a parameter that can be controlled externally, and a large $\beta_{\mathrm{L}}$ results in more triangular oscillations. If one junction is topologically trivial, the same effect occurs, combined with a phase shift due to asymmetry between the junctions. Therefore, even in rings including topologically trivial components, a $\sin (\phi / 2)$ current-phase relationship can be detected, although the effect is more subtle than a $2 \Phi_{0}$ periodicity.

\subsubsection{Topologically non-trivial SQUIDs}

In the case when the ring is completely topologically non-trivial, corresponding to Fig. 6.Ib, $2 \Phi_{0}$ periodicity is to be observed. In the limiting case $\beta_{\mathrm{L}}=0$ and $\mathrm{I}=\mathrm{I}_{\mathrm{c}} \sin (\phi / 2)$, the critical current dependence on field can be written as $\mathrm{I}=2 \mathrm{I}_{\mathrm{c}}\left|\cos \left(\pi \frac{\Phi}{2 \Phi_{0}}\right)\right|$, resulting in the $2 \Phi_{0}$ periodicity. If both $\sin (\phi)$ and $\sin (\phi / 2)$ components are present in the junctions, both periodicities are observed, as shown in Fig. 6.3a for equal ratios in the junctions. Interestingly, increasing $\beta_{\mathrm{L}}$ results in a larger $2 \Phi_{0}$ component and a reduced $\Phi_{0}$ component. In Fig. 6.3b the ratio dependence on $\beta_{\mathrm{L}}$ is shown, where the ratio is defined using the frequency amplitude after Fourier transformation. The screening parameter $\beta_{\mathrm{L}}$ is composed of the critical current of the junctions, and the inductance of the ring determined by geometrical factors, but is also dependent on the charge carrier in the ring. Since Majorana tunnelling is with charge $e$ instead of $2 e$ as is the case for Cooper pair transport, the effective screening is reduced by a factor 2. By optimizing $\beta_{\mathrm{L}}$ using the tunable inductance of the ring, it is therefore possible to dramatically increase the $2 \Phi_{0}$ component relative to the standard $\Phi_{0}$-periodic component, ideal for the observation of the Majorana fermion. 

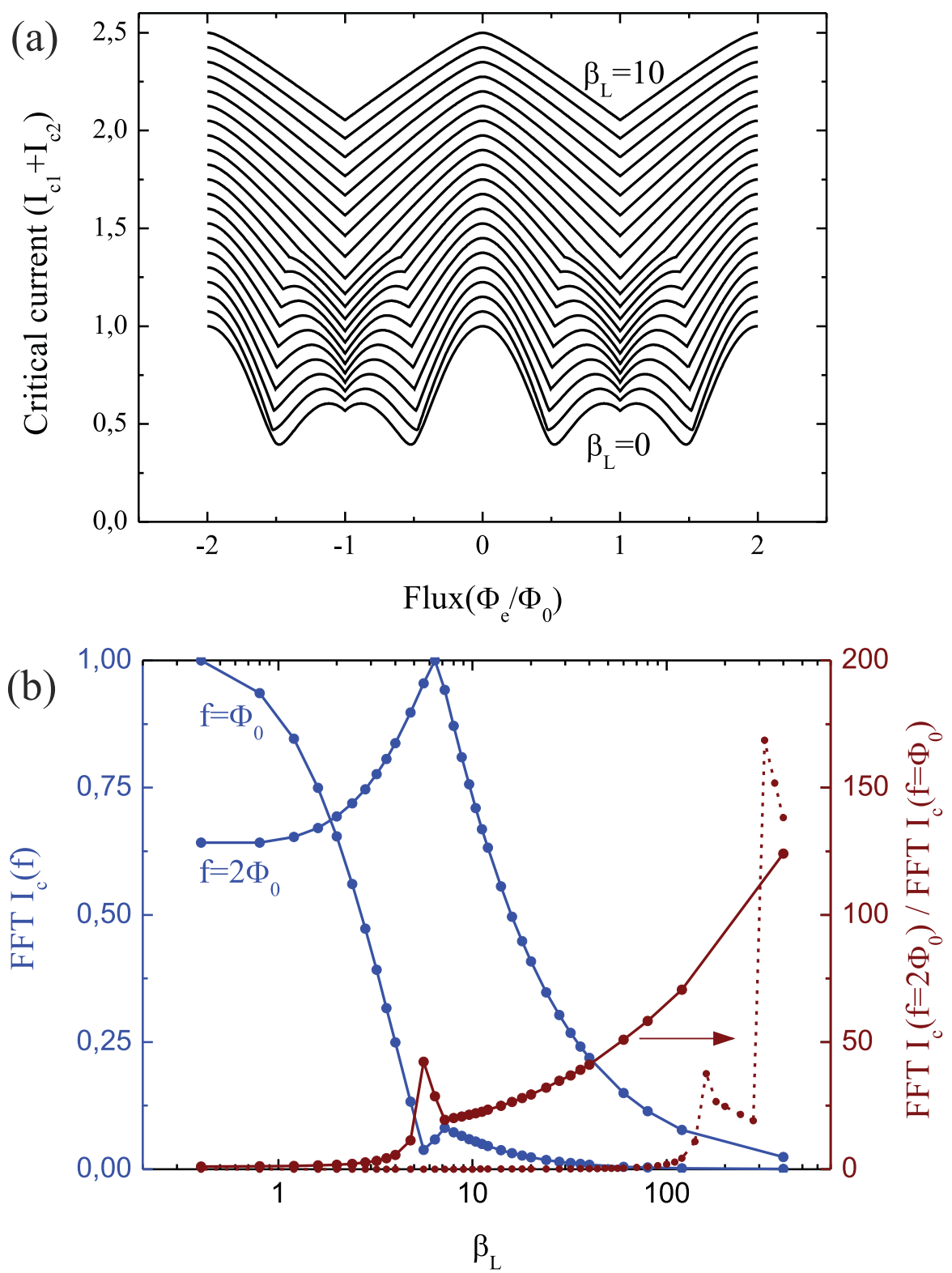

Figure 6.3: CURRENT-PHASE CHARACTERISTICS OF A DC SQUID COMPOSED OF A TOPOLOGICALLY NON-TRIVIAL SUPERCONDUCTING RING AND NON-TRIVIAL JUNCTIONS. (a) dc SQUID oscillations for two symmetric junctions with equal amplitude $\sin (\phi)$ and $\sin (\phi / 2)$ components, $\alpha=0.5$. Increasing $\beta_{\mathrm{L}}$ (in steps $\delta \beta_{\mathrm{L}}=0.5$, and shifted for clarity) promotes the $2 \Phi_{0}$ period, since the effective screening is smaller for Majorana tunnelling than Cooper pair tunnelling. (b) (blue) FFT amplitude of the two components as function of $\beta_{\mathrm{L}}$. (red) Evolution of the FFT amplitude ratio as a function of $\beta_{\mathrm{L}}$. The dashed lines represent the ratio when the junctions have only $5 \% \sin (\phi / 2)$ component contribution. The $2 \Phi_{0}$ component can be more than 2 orders of magnitude larger than the $\Phi_{0}$ component. 
(a)

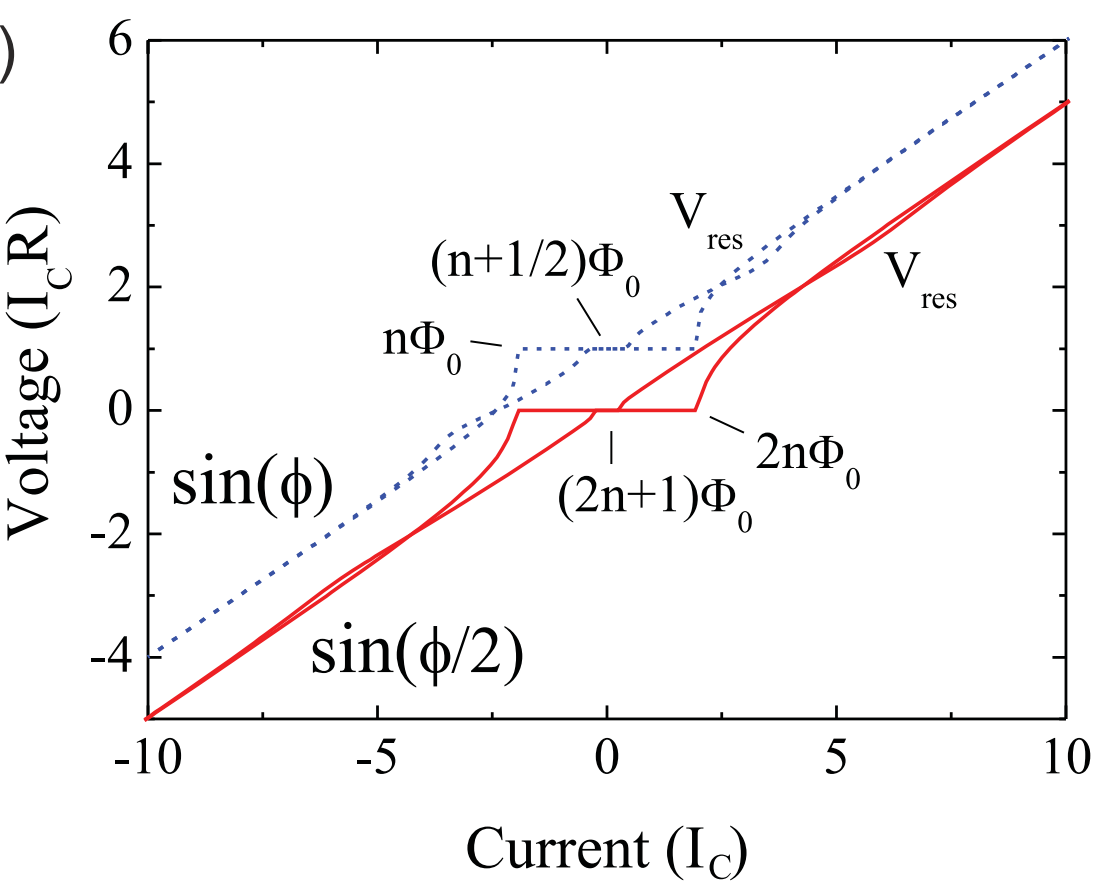

(b)

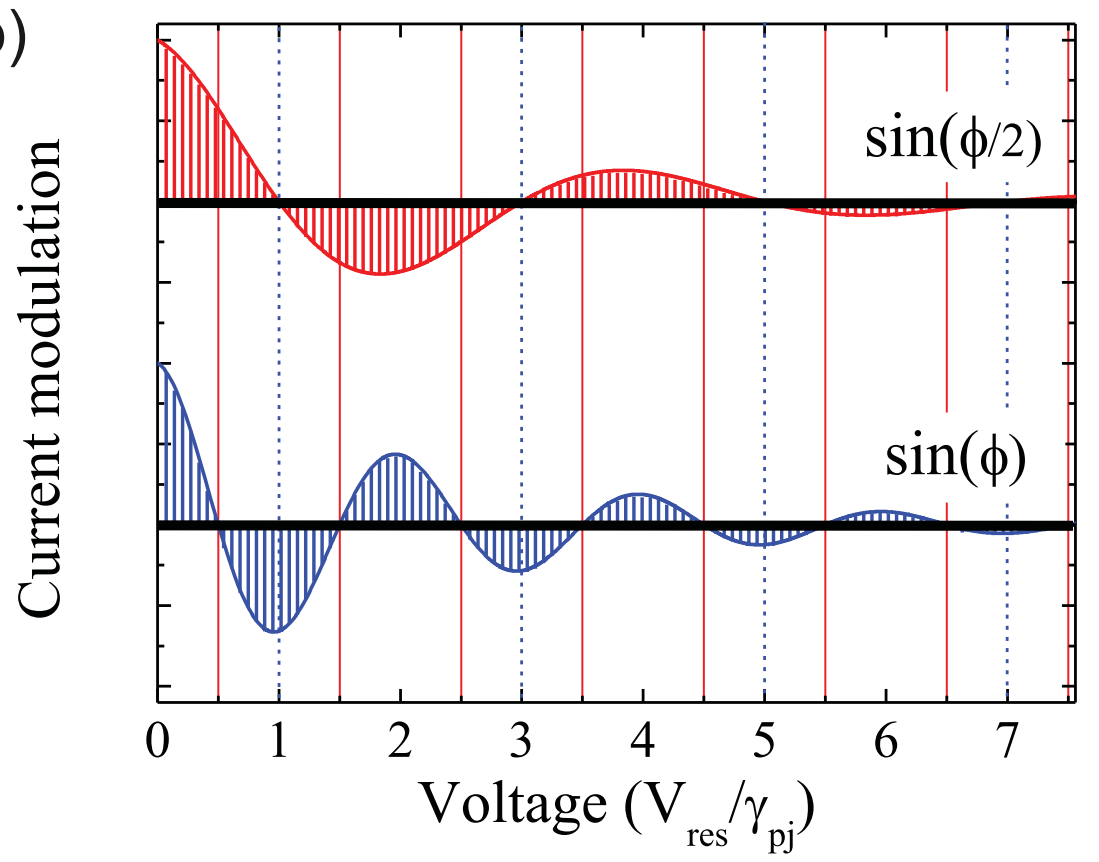

Figure 6.4: VOLTAGE CHARACTERISTICS OF NON-TRIVIAL DC SQUIDS WITH MIXED JUNCTIONS. (a) dc SQUID IV characteristics for junctions with $\sin (\phi)$ and $\sin (\phi / 2)$ current-phase relationships; $\beta_{C}=1, \beta_{L}=1$. The $\sin (\phi / 2)$ (solid line) component doubles the resonance voltage $V_{\text {res }}$ (dashed line) with respect to the standard SQUID. The IV characteristic of the standard SQUID is shifted for clarity. (b) Damping and resonance in the SQUID. The $\sin (\phi / 2)$ component oscillates with half the frequency, so that the $\sin (\phi)$ and $\sin (\phi / 2)$ components have their minima and maxima at different voltages (solid red are maxima for the $\sin (\phi / 2)$ component and dashed blue maxima for the $\sin (\phi)$ component. 


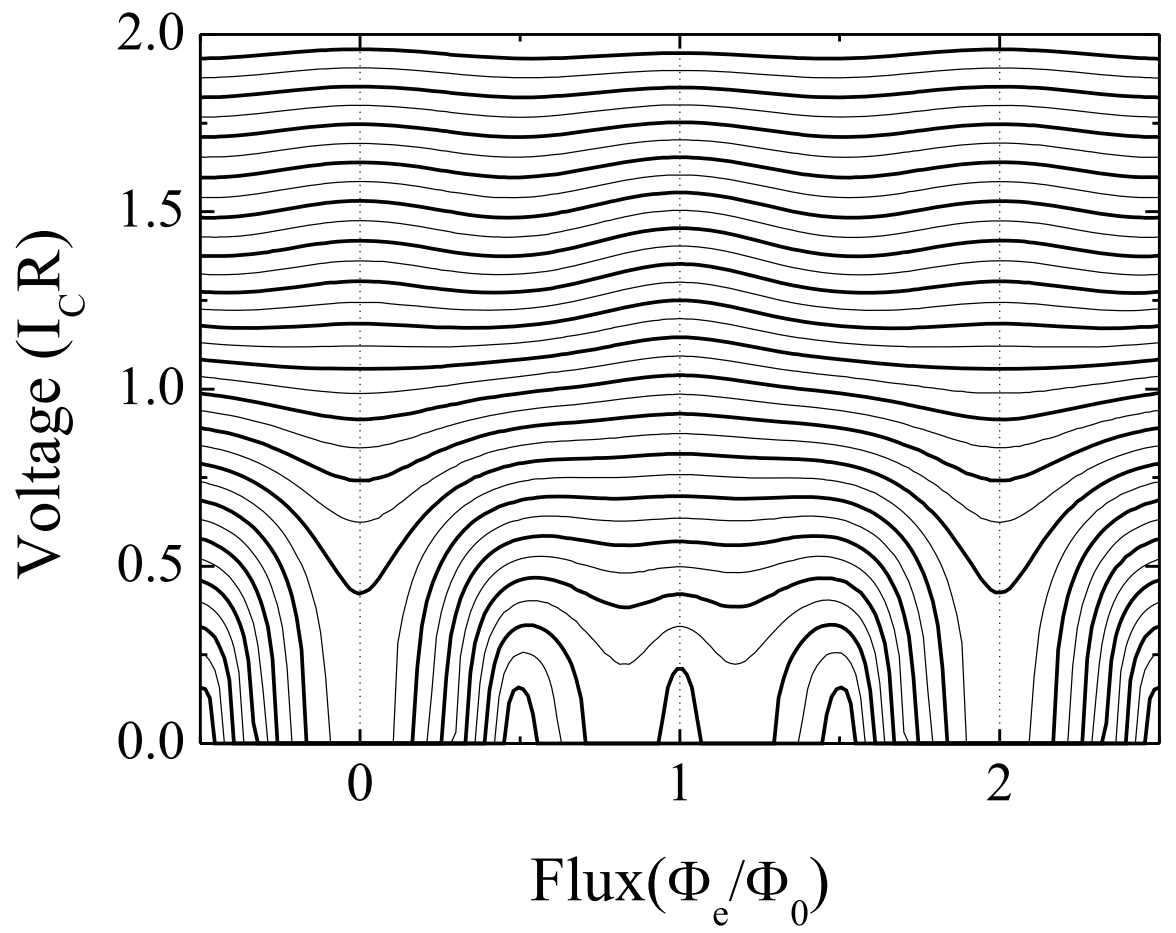

Figure 6.5: TOPOLOGICALLY NON-TRIVIAL DC SQUID IN THE VOLTAGE MODE. $V \phi$ characteristics of a dc SQUID with equal $\sin (\phi)$ and $\sin (\phi / 2)$ components in the junctions, and $\beta_{\mathrm{C}}=1, \beta_{\mathrm{L}}=1$. The SQUID shows both $\Phi_{0}$ and $2 \Phi_{0}$ periodicity, depending on the voltage close to $V_{\text {res, } \phi}$ or $V_{\text {res, } \phi / 2}$, which is controlled by the bias current. 


\subsubsection{The voltage state}

When the SQUID is operated in the voltage mode, both $\Phi_{0}$ and $2 \Phi_{0}$ periodicity can be observed, even if the ring includes trivial components. The relative amplitude depends on the voltage, controlled by the bias current. Figure 6.4a shows the IV characteristics for a dc SQUID with either pure $\sin (\phi)$ or $\sin (\phi / 2)$ components. The current modulation for high voltages is inverted with respect to the modulation for small voltages. This is the result of the nonlinear interaction of the ac Josephson current with the resonant circuit formed by the loop inductance $\mathrm{L}$ and the junction capacitance $C$. This resonance voltage is given by $V_{\text {res }}=\gamma_{p j} \sqrt{\frac{2}{\beta_{C} \beta_{L}}} I_{c} R$. As a result, the $\sin (\phi)$ and $\sin (\phi / 2)$ components cause oscillations with different frequency as a function of voltage, see Fig. 6.4b. Consequently, the relative amplitude of the $2 \pi$ and $4 \pi$ periodicity of the $\mathrm{V}(\phi)$ characteristics, shown in Fig. 6.5 for $\beta_{C}=1$, and $\beta_{L}=1$ depend on the resonance voltages $V_{\text {res, } \phi}$ and $V_{\text {res }, \phi / 2}$, which is controlled by the bias current. The resonance voltage is independent of the loop parity, but for $\gamma_{\mathrm{pl}}=1$ the oscillation amplitude is reduced by quantum phase slips. This is similar to the cause of the incomplete critical current modulation shown in Fig. 6.2.

The damping of the current modulation with increasing bias voltage is characterised by $\zeta=\sqrt{\frac{2 \beta_{C}}{\beta_{L}}}$, independent on the current-phase periodicity, and SQUIDs with $\sin (\phi / 2)$ component junctions have therefore a smaller current modulation where the modulation is inverted, compared to standard SQUIDs. Choosing a large $\beta_{\mathrm{L}}$ will decrease the damping term and increase the amplitude of the voltage resonances. A small $\beta_{C}$ will reduce the damping term but increase the resonance voltage.

\subsection{APPLICATIONS TO TOPOLOGICALLY NON-TRIVIAL SYS- TEMS}

We now discuss the implications of our proposals. The possible realisation of a new emergent particle in condensed matter physics together with the potential for quantum computation, boosted the search for superconducting systems hosting the Majorana fermion. Superconductor - semiconductor structures in the presence of strong spin orbit coupling and Zeeman fields are currently quite successful and signatures of Majorana fermions have been observed, characterised by zero bias 
conductance peaks [23]. In superconductor - topological insulator junctions, Josephson supercurrents have also been observed [19-21, 30]. In the case of topological insulator systems, bulk shunting likely introduces $\sin (\phi)$ terms in the current-phase relation. Quantum phase slips [17] and quasiparticle poisoning [16] will be relevant for all proposals, relaxing the system to $\sin (\phi)$ periodicity. Therefore, increasing the $\sin (\phi / 2)$ component is important for all these proposals.

The parameter $\beta_{\mathrm{L}}$ is easily tunable. This SQUID parameter depends on the inductance, determined by the SQUID geometry, and independent of the individual junctions. The parameter $\beta_{C}$ is a junction parameter and therefore more difficult to tune. However, varying the length and width of the junctions and controlling the interface transparency tune $\beta_{C}$.

\subsection{CONCLUSIONS}

In conclusion, we have studied dc SQUIDs containing two topologically non-trivial barriers. The $2 \Phi_{0}$ periodicity stemming from the Majorana fermion can only be detected in non-trivial dc SQUIDs. However, even loops containing topologically trivial elements are influenced by the presence of junctions with $\sin (\phi / 2)$ components. This is observed both in the critical current modulation by flux and the resonance voltage. The SQUID parameters can be used to increase the relative component; increasing $\beta_{\mathrm{L}}$ is found to largely increase the component with the largest periodicity. The $\mathrm{V}(\phi)$ relation is altered when both components are present, and both components can be maximised at different bias currents, determined by the resonance voltage. In recently fabricated S-TI-S junctions, $\beta_{C}$ is usually low due to bulk shunting. This increases the resonance voltage to a regime where the damping is higher, which complicated observing a clear difference between $\Phi_{0}$ and $2 \Phi_{0}$ periodicities. Decreasing this bulk shunt would therefore simplify the $2 \Phi_{0}$ periodicity observation. Nonetheless, the strong effect of $\beta_{\mathrm{L}}$, large $\beta_{\mathrm{L}}$ increases the $2 \Phi_{0}$ component over 100 times, allows the detection of the Majorana fermion, under the right intrinsic circumstances (e.g. no relaxation to equilibrium), in dc SQUIDs composed of present day S-TI-S junctions. This result is also of relevance to devices where the Majorana character is induced via other means, such as in semiconducting nanowires with strong Rashba spin orbit coupling. Tuning $\beta_{\mathrm{L}}$ in combination with ac 
measurements to prevent relaxation paves the way to the observation of exotic properties of the Majorana fermion.

\section{BIBLIOGRAPHY}

[1] L. Fu and C. L. Kane, Phys. Rev. Lett. 100, 096407 (2008).

[2] Y. Tanaka, T. Yokoyama, and N. Nagaosa, Phys. Rev. Lett. 103, 107002 (2009).

[3] B. A. Bernevig, T. L. Hughes, and S.-C. Zhang, Science 314, 1757 (2006).

[4] L. Fu, C. L. Kane, and E. J. Mele, Phys. Rev. Lett. 98, 106803 (2007).

[5] H. Zhang, C.-X. Liu, X.-L. Qi, X. Dai, Z. Fang, and S.-C. Zhang, Nat Phys 5, 438 (2009).

[6] X.-L. Qi, R. Li, J. Zang, and S.-C. Zhang, Science 323, 1184 (2009).

[7] D. Hsieh, D. Qian, L. Wray, Y. Xia, Y. S. Hor, R. J. Cava, and M. Z. Hasan, Nature 452, 970 (2008).

[8] Y. L. Chen, J. G. Analytis, J.-H. Chu, Z. K. Liu, S.-K. Mo, X. L. Qi, H. J. Zhang, D. H. Lu, X. Dai, Z. Fang, S. C. Zhang, et al., Science 325, 178 (2009).

[9] D. Hsieh, Y. Xia, D. Qian, L. Wray, J. H. Dil, F. Meier, J. Osterwalder, L. Patthey, J. G. Checkelsky, N. P. Ong, A. V. Fedorov, et al., Nature 460, 1101 (2009).

[10] H. Peng, K. Lai, D. Kong, S. Meister, Y. Chen, X.-L. Qi, S.-C. Zhang, Z.-X. Shen, and Y. Cui, Nat Mater 9, 225 (2010).

[11] P. Cheng, C. Song, T. Zhang, Y. Zhang, Y. Wang, J.-F. Jia, J. Wang, Y. Wang, B.-F. Zhu, X. Chen, X. Ma, et al., Phys. Rev. Lett. 105, 076801 (2010).

[12] T. Zhang, P. Cheng, X. Chen, J.-F. Jia, X. Ma, K. He, L. Wang, H. Zhang, X. Dai, Z. Fang, X. Xie, et al., Phys. Rev. Lett. 103, 266803 (2009).

[13] J. D. Sau, R. M. Lutchyn, S. Tewari, and S. Das Sarma, Phys. Rev. Lett. 104, 040502 (2010).

[14] J. Alicea, Phys. Rev. B 81, 125318 (2010). 
[15] E. Majorana, Il Nuovo Cimento 14, 171 (1937).

[16] L. Fu and C. L. Kane, Phys. Rev. B 79, 161408 (2009).

[17] C. Beenakker, Annual Review of Condensed Matter Physics 4, 113 (2013).

[18] D. M. Badiane, M. Houzet, and J. S. Meyer, Phys. Rev. Lett. 107, 177002 (2011).

[19] D. Zhang, J. Wang, A. M. DaSilva, J. S. Lee, H. R. Gutierrez, M. H. W. Chan, J. Jain, and N. Samarth, Phys. Rev. B 84, 165120 (2011).

[20] B. Sacépé, J. B. Oostinga, J. Li, A. Ubaldini, N. J. Couto, E. Giannini, and A. F. Morpurgo, Nat Communications 2, 575 (2011).

[21] M. Veldhorst, M. Snelder, M. Hoek, T. Gang, V. K. Guduru, X. L. Wang, U. Zeitler, W. G. van der Wiel, A. A. Golubov, H. Hilgenkamp, and A. Brinkman, Nature Materials 11, 417 (2012).

[22] M. Veldhorst, C. G. Molenaar, X. L. Wang, H. Hilgenkamp, and A. Brinkman, Applied Physics Letters 100, 072602 (2012).

[23] V. Mourik, K. Zuo, S. M. Frolov, S. R. Plissard, E. P. A. M. Bakkers, and L. P. Kouwenhoven, Science 336, 1003 (2012).

[24] A. A. Golubov, M. Y. Kupriyanov, and E. Il'ichev, Rev. Mod. Phys. 76, 411 (2004).

[25] K. Kang, EPL (Europhysics Letters) 51, 181 (2000).

[26] F. Loder, A. P. Kampf, and T. Kopp, Phys. Rev. B 78, 174526 (2008).

[27] L. Fu, Phys. Rev. Lett. 104, 056402 (2010).

[28] B. van Heck, F. Hassler, A. R. Akhmerov, and C. W. J. Beenakker, Phys. Rev. B 84, 180502 (2011).

[29] C. Tesche and J. Clarke, Journal of Low Temperature Physics 29, 301 (1977).

[30] F. Qu, F. Yang, J. Shen, Y. Ding, J. Chen, Z. Ji, G. Liu, J. Fan, X. Jing, C. Yang, and L. Lu, Sci. Rep. 2 (2012). 


\section{CRITICAL BEHAVIOUR AT THE DYNAMIC MOTT TRANSITION}

In a superconducting array of niobium islands on a gold surface we have realised the vortex equivalent of an electron Mott insulator. Control over the magnetic field controls the vortex density and accesses fractional Mott insulator states. The role of a bias current in a vortex system is dual to the role of electric potential in an electron system, and induces an insulator to metal transition. The critical exponent describing the phase transition of the integer vortex Mott insulator is that of a temperature-driven Mott transition. For the fractional Mott insulator state a different critical exponent is found, reflecting the anti-ferromagnetic ordering of vortices compared to the ferromagnetic ordering of the integer state.

\subsection{INTRODUCTION}

Mott insulators $[1-3]$ are at the heart of the physics of strongly correlated systems [4], encompassing phenomena and systems that range from high temperature superconductivity [5] and quantum transitions of ultracold atomic gases [6-12] to quantum spin liquid states [13]. Furthermore, there is an increasing interest in the Mott insulator-to-metal transition induced by applied electric fields, as an exemplary representative of a nonequilibrium phase transition [14-16]. However, interfering effects such as disorder, environmental noise and an imprecise control over external drives, impede the understanding of the fundamental mechanisms governing nonequilibrium phase transitions and their respective drives.

Using a square proximity array of superconducting islands placed on a normal metal, we have created a vortex Mott insulator which offers a unique opportunity for control of the tuneable parameters of Mott phase and its dynamic state. We discover a current-induced vortex Mott transition and find a critical scaling $|i| \propto|h|^{2 / 3}$, where $i$ and $h$ are the respective de- 
viations from the transition current and the matching magnetic fields at which the vortex Mott insulator forms.

The critical exponent exhibits a striking similarity with the scaling of the equilibrium electronic temperature-driven Mott transition [17], $|t| \propto|p|^{2 / 3}$, where $t$ and $p$ are the deviations from the temperature and applied pressure, controlling parameters of the transition, from their critical values. This establishes a deep connection between the thermodynamic phase transitions governed by the temperature and its out-of-equilibrium dynamic counterpart induced by applied current.

In the same superconducting array we observe novel fractional vortex Mott insulating states at partial fillings of pinning sites as a dual to fractional quantum Hall electronic states. We observe strikingly exhibit different critical exponents governing the dynamic phase transitions of these fractional states. Our results mark an important step towards an understanding of the physics governing transitions between equilibrium and non-equilibrium states. As well as that, these results offer a new experimental tool for controlling fractional Mott states and investigating their dynamic phase transitions.

\subsection{MOTT PHENOMENA AND TRANSITION}

A deep understanding of the Mott transition is the key ingredient for a wide range of physical phenomena, such as unravelling the origin of high temperature superconductivity, the quantum phase transition in a Bose-Einstein condensate [8], and as a base for metal-insulator-semiconductor field-effect transistors and related applications [18]. The field of phase transitions in strongly correlated systems is growing with the study of nonequilibrium phase transitions. Potential induced insulator to metal transitions have been described theoretically using several approaches $[16,19,20]$. Phase transitions have been observed in one-dimensional [14] and narrow band [15] Mott insulators, and a potential gradient was studied to probe the excitation spectrum in a cold atom gas [8].

A general approach to dynamic phase transitions was taken by Chtchelkatchev et al. [21], where a non-equilibrium meanfield Ginzburg-Landau (GL)-type theory was constructed for an electronic Mott insulator. The main result obtained in the context of fluctuation superconductivity was that the nonequilibrium order parameter vanishes as $\sqrt{\left|V-V_{c}\right|}$ in the out-ofequilibrium state, $\mathrm{V}$ being a voltage bias, replacing the cus- 
tomary $\sqrt{\left|T_{c}-T\right|}$-dependence of the equilibrium state. A different realisation of a Mott system is a vortex Mott insulator [22], where there is a mathematical mapping between $3 \mathrm{D}$ vortices and the strongly repulsive charges of an electronic Mott insulator. According to vortex-charge mapping, the applied current plays the role of the electric field, and the dynamic resistance, $\mathrm{dV} / \mathrm{dI}$, probes the density of the mobile vortex states as a mirror image to dynamic conductivity, $d I / d V$, which measures the density of the electronic states in electronic Mott insulators [24, 25]. Since the derivation by Chtchelkatchev et al. [21] was done on a generic symmetry ground without assuming any specifics of a particular system, we make a general conjecture that in a mean field approximation the duality between the equilibrium and non-equilibrium phase transitions manifests itself through temperature $\leftrightarrow$ external drive mapping. In the case of a dynamic vortex Mott transition we thus expect $\left|\mathrm{T}-\mathrm{T}_{\mathrm{c}}\right| \leftrightarrow\left|\mathrm{I}-\mathrm{I}_{0}\right|$ correspondence. Upon increasing the current the critical scaling of dynamic magnetoresistance is revealed which surprisingly coincides exactly with the scaling of the equilibrium Mott transition [17] up to the correspondence current $\leftrightarrow$ temperature. Our observation establishes a unifying connection between equilibrium and dynamic phase transitions as well as a duality between vortex and quantum strongly correlated systems.

\subsection{SAMPLE DESIGN AND FABRICATION*}

To realise a vortex Mott insulator we prepared a proximity array which localises vortices at the energy dimples of an egg crate potential [23]. Standard photolithographic tools have been employed to create a $40 \mathrm{~nm}$ thick Au template (see Fig. 7.I) on a $\mathrm{Si} / \mathrm{SO}_{2}$ substrate of $1 \times 1 \mathrm{~cm}^{2}$. The template consists of a central square of $82 \times 82 \mu \mathrm{m}^{2}$, with the corners connected to 4 terminals in a Van der Pauw configuration for electrical characterisation. The size of the terminals is $200 \times 200 \mu \mathrm{m}^{2}$, large enough for $\mathrm{mi}$ cro bonding. The Au pattern is overlaid with a square array of superconducting niobium $(\mathrm{Nb})$ islands $45 \mathrm{~nm}$ thick. The $\mathrm{Nb}$ pattern is created on top of the central Au square employing electron beam lithography and DC sputtering, via a standard PMMA lift off procedure. Transport between the $\mathrm{Nb}$ islands can take place via the Au template that lies underneath. The result-

\footnotetext{
*Sample fabrication by F. Coneri and N. Poccia.
} 
a
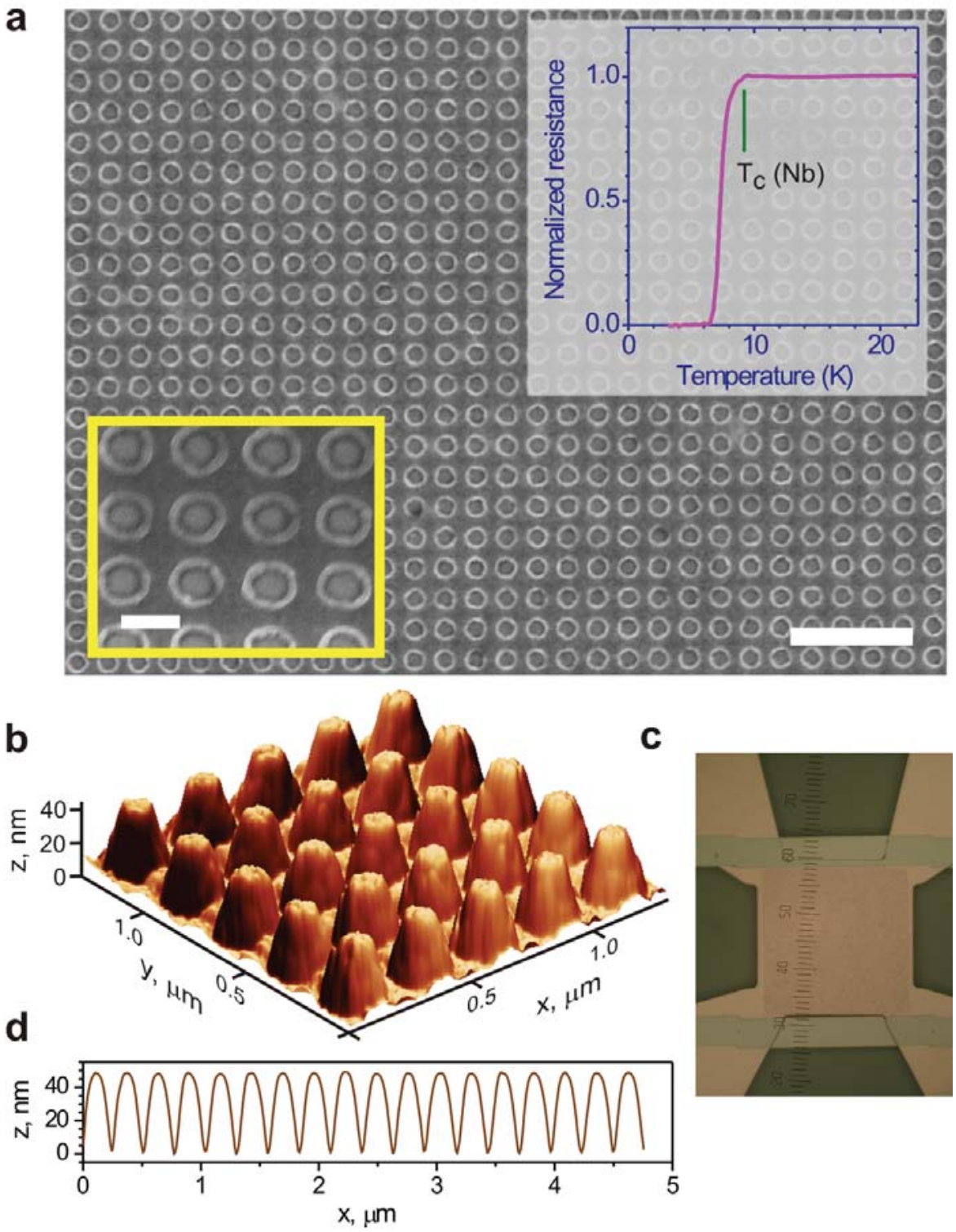

Figure 7.1: EXPERIMENTAL SET UP. (a) Scanning electron microscopy (SEM) image of square array of $\mathrm{Nb}$ islands on $\mathrm{Au}$. The scale bar is $1 \mu \mathrm{m}$. The bottom-left inset shows a magnified image of the array (the scale bar is $200 \mathrm{~nm}$ ). The topright inset shows the temperature dependence of the resistance near the superconducting transition. The superconducting critical temperature of bulk $\mathrm{Nb}$ is marked by the vertical bar. (b) $1.4 \times 1.4 \mu \mathrm{m}^{2}$ AFM topography of the superconductor islands on the metallic template of the same sample. (c) Optical image of the Au template with four contacts (yellow), $\mathrm{Nb}$ bus bars (light green), the array of $300 \times 300 \mathrm{Nb}$ islands barely visible as a red square shadow on a $\mathrm{Si} / \mathrm{SiO}_{2}$ substrate (dark green). (d) Maximum height profile along the principle axis of the array. 
ing $\mathrm{Nb}$ array is $80 \times 80 \mu \mathrm{m}^{2}$ large and comprises $300 \times 300$ sites. The 90,000 $\mathrm{Nb}$ islands are placed with a period $\mathrm{a}=267 \mathrm{~nm}$. The diameter of an island is $220 \pm 3 \mathrm{~nm}$ and the island separation is $47 \pm 3 \mathrm{~nm}$. Panels a-d of Fig. 7.I show scanning electron, atomic force microscopy, and optical images of the sample and the height profile along one of the principal axis of the array.

\subsection{DEVICE CHARACTERISATION}

At zero field, the temperature and bias dependence of a superconducting array is that of a BKT transition [27-29]: vortexantivortex pairs are unbound by either temperature or current, and in turn contribute to dissipation. A background magnetic field linearly controls the number of vortices in the system and hence controls the (fractional) Mott phase, going well beyond the regime of BKT physics. These fractional Mott phases are formed when vortices can arrange themselves periodically in the egg-crate potential provided by the superconducting island lattice. Careful control over the driving force enables us to closely follow the evolution of the Mott phase in the critical region.

We first investigate the temperature dependence of the array resistance. Four-point probe resistivity measurements to determine the transition temperature are performed in a Quantum Design PPMS system, warming from $1.9 \mathrm{~K}$ at a rate of $0.3 \mathrm{~K} / \mathrm{min}$. The superconducting transition temperature of the array, determined as the midpoint of the temperature resistance curve in the upper inset in Fig. 7.1a, is $T_{c}=7.3 \mathrm{~K}$ which is $2 \mathrm{~K}$ lower than $T_{c}=9.3 \mathrm{~K}$ of bulk niobium. This moderate downward shift of the transition temperature implies that the array is a strongly coupled network of superconducting islands [26].

In order to identify the critical behaviour of the dynamic Mott transition we perform transport measurements in the currentvoltage-magnetic field ( $\mathrm{I}, \mathrm{V}, \mathrm{B})$ space scanning I and $\mathrm{B}$ in small steps. To probe the density of mobile vortices the differential resistance, $d V / d I$, is used $[24,25]$. To ensure a uniform current injection into the array during magnetoresistance measurements, two $45 \mathrm{~nm} \mathrm{Nb}$ bus bars are deposited along two opposite sides of the array, as shown in Fig.7.1c; the gap between strips and array is less that $0.5 \mu \mathrm{m}$. When cooled below $9 \mathrm{~K}$, the bars become superconducting and effectively short the corresponding current and voltage leads. This results in a 2-point measurement that yields magnetoresistance data shown in Fig. 7.2. The 
IV measurements are carried out in a shielded cryostat at $1.4 \mathrm{~K}$. A current bias is applied using a ramp generator at several $\mathrm{Hz}$. The measured voltage waveform is amplified using homebuilt low-noise electronics, and subsequently digitised with a National Instruments PXI-4461. The IV characteristics are nonhysteretic and averaged over several current cycles. The dynamic resistance is found by numerically differentiating the averaged IV characteristics. A magnetic field is applied by placing a solenoid around the sample with the field perpendicular to the sample plane. The current through the solenoid increases in a stepwise fashion and separate IV traces are recorded at each field step. The obtained IV curves are nonlinear, similar to superconducting junction characteristics but with a residual resistance less than $50 \mathrm{mOhm}$. This resistance corresponds to the finite distance between the superconducting bus bars and the $\mathrm{Nb}$ island array.

Panel a of Fig. 7.2 shows colour plots of the differential resistance as function of the applied current and the magnetic field in units of the frustration parameter $f=B / B_{0}$, where $\mathrm{B}_{0}=\Phi_{0} / \mathrm{a}^{2}$, and $\Phi_{0}=\pi \hbar / e$ is the magnetic flux quantum. In our array $\mathrm{B}_{0}=28.6 \mathrm{mT}$. The data presented in Fig. 7.2 a enable a meaningful scaling analysis which will reveal the nature of the Mott insulator-to-metal transition in the dynamic regime. To highlight the transition we display isocurrent cuts as $d V / d I$ vs. B plots in Fig. 7.2b. At modest currents we observe the expected dips in $\mathrm{dV} / \mathrm{dI}$, or equivalently minima in the density of states, at rational $f=f_{c} \equiv p / q$ reflecting modulation of the ground state energy $E_{g}$ and formation of periodic vortex patterns in a magnetic field (Fig. 7.2c,d). The $E_{g}(f)$ dependence is given by the minimum energy as function of field of the Harper equation spectrum $[31,32]$. This is the same spectrum that corresponds to tight-binding electrons on a two-dimensional square lattice under a magnetic field perpendicular to the plane and which is a signature of a fractional quantum Hall effect [33], and a manifestation of the duality between vortices and electrical charges [22, 34]. Viewing Fig. $7.2 \mathrm{~d}$ as a visualisation of the Mott-Hubbard model for electrons the case $f=1$ would correspond to a Mott insulator. Accordingly, for its vortex counterpart, the case of complete filling of the pinning sites array $\left(f_{c}=1,2, \ldots\right)$ was conjectured to form a vortex Mott insulator [22]. Our data enable us to extend this conjecture onto fractional filling and propose a fractional Mott insulator, a mirror 

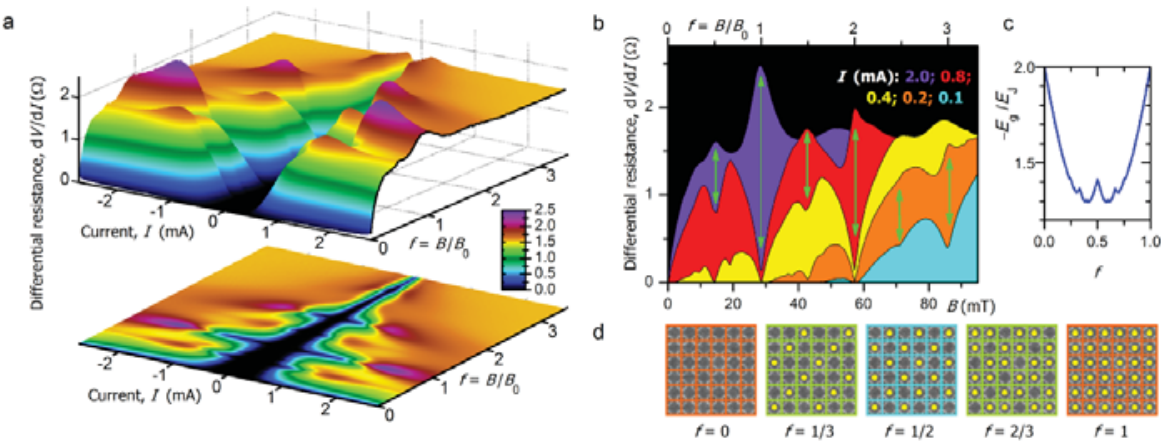

Figure 7.2: Vortex Mott insulator-to-Metal transition. a: Color plots of the differential resistance as function of the applied current and magnetic field in units of $\mathrm{B}_{0}=$ $\pi \hbar /\left(e a^{2}\right)=28.6 \mathrm{mT}$. The color bar is given in Ohms. b: Representative differential resistances as functions of the frustration factor $f=B / B_{0}$ taken at different bias currents. At low current bias (blue, orange, and yellow), the differential resistance minima at commensurate $f=1 / 3,1 / 2,2 / 3,1,4 / 3,3 / 2,5 / 3,2,7 / 3,5 / 2,8 / 3,3$ are visible. Increasing the current flips the minima into maxima (red and violet). It manifests the Mott-insulator-to metal transitions and is highlighted by vertical arrows (the details of the insulator-to metal evolution at $f=1 / 2,1,2$ are shown below in the upper row of Fig. 7.4). c: Energy of the ground state as function of $f \in[0,1]$ obtained by solving the Harper equation [32]. (d) Vortex configurations at commensurate frustration factor $f=0,1 / 3,1 / 2,2 / 3,1$; at integer $f$ the conventional vortex Mott insulator forms, partial fillings correspond to fractional Mott insulator states. 
image of fractional quantum Hall states. The profound low current minima in $d V / d I$ at rational $f$ support this conclusion.

Our key finding is that the transition from the Mott insulating to metallic state manifests itself as a reversal of the minima in $\mathrm{dV} / \mathrm{dI}$ at rational $\mathrm{f}_{\mathrm{c}}$ into maxima upon increasing the current bias, indicating the population of free vortex states. The effect is most pronounced at integer and half-integer $f$, although at $f=1 / 3,2 / 3, \ldots$ the $d V / d I$ flips are also still distinguishable (Fig. 7.2b). Note that the observed dip-to-peak evolution upon increasing current exists only in $\mathrm{dV} / \mathrm{dI}$, but not in the $\mathrm{R}=\mathrm{V} / \mathrm{I}$ quantity, see figure 7.3. The absence of the peak in $R$ implies that depinning of vortices at elevated currents, which would have resulted in the dramatic increase of dissipation, cannot explain the observed evolution in $\mathrm{dV} / \mathrm{dI}[35,36]$.

\subsection{SCALING ANALYSIS ${ }^{\dagger}$}

We present detailed plots of $d V / d I$ vs. B curves in the vicinity of $f_{c}=1 / 2,1,2$ in the critical bias region in the upper row panels of Fig. 7.4. There is an asymmetry between the left-hand-side and right-hand-side vicinities of the commensurability points which we consider independently. In either of them there is an apparent separatrix between insulating-like and metallic-like regions. We identify the critical currents $\mathrm{I}_{0}^{ \pm}$, corresponding to this divide between insulating and metallic behaviour from the condition $d(d V / d I) /\left.d f\right|_{f=f_{c}^{ \pm}}=0$, as currents at which the dynamic Mott transition occurs. These separatrices are highlighted by dots in Fig. 7.4a-c. For $\mathrm{I}>\mathrm{I}_{0}^{ \pm}$the $\mathrm{dV} / \mathrm{dI}$ traces display minima at $\left|f-f_{c}\right|>0$, marked by strokes in Fig. 7.4a-c, encompassing the region of metallic behaviour. These critical regions widen upon increasing current similar to the standard quantum phase transition critical region which increases with growing temperature [37].

Now we demonstrate that $\left(I_{0}, f_{c}\right)$ is indeed the Mott transition point that exhibits critical behaviour and we determine the corresponding critical exponents. A scaling theory states that in the critical region of a continuous phase transition physical observables follow the scaling laws, so that their respective correlation lengths diverge on approach to the critical point [38, 39]. The Mott insulator-to-metal transition is driven by tuning the ratio $\mathrm{U} / \mathrm{J}$ between the repulsion strength $\mathrm{U}$ and the character-

\footnotetext{
${ }^{\dagger}$ Key ideas proposed by T. I. Baturina and V. M. Vinokur.
} 

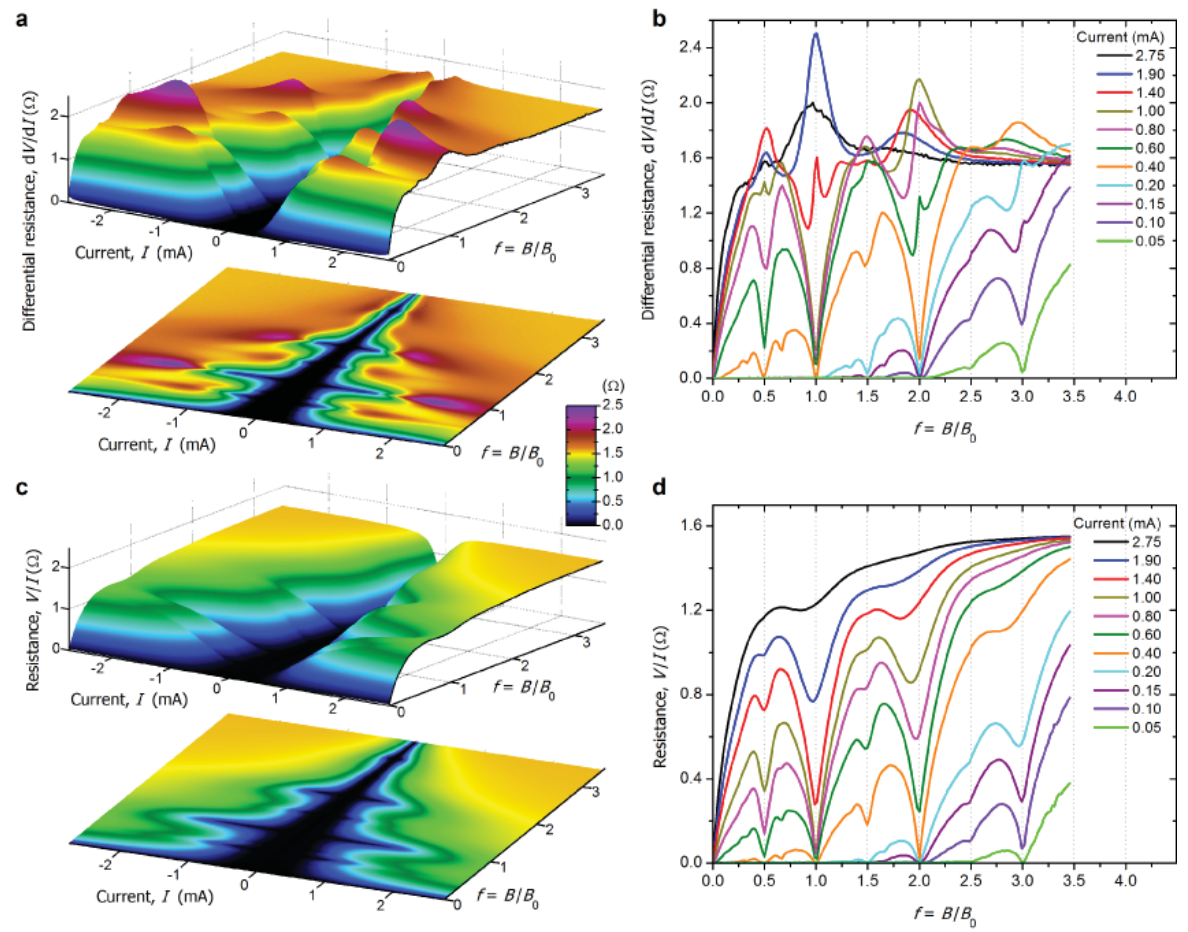

Figure 7.3: DifFERENTIAL RESISTANCE AND RESISTANCE AS FUNCTION OF FRUSTRATION. (a) and (c): colour plots of differential resistance and resistance. Colour scales are the same for both cases. (b) and (d): representative curves of differential resistance and resistance as functions of the frustration factor $\mathrm{f}=\mathrm{B} / \mathrm{Bo}$. Both panels present the curves measured at the same dc currents, which are denoted by the same colours. The dip-to-peak evolution at rational $\mathrm{f}$ upon increasing the current is experienced only by the differential resistance, $\mathrm{dV} / \mathrm{dI}$, but not by the resistance, $\mathrm{V} / \mathrm{I}$. 

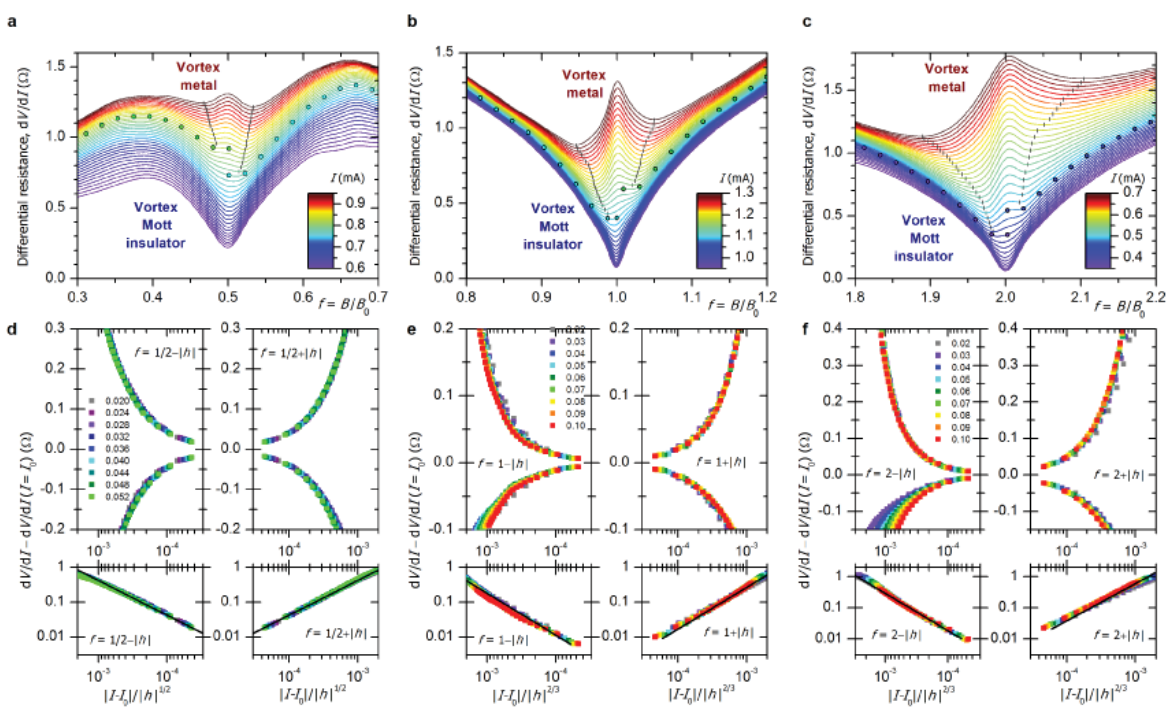

Figure 7.4: SCALING AT THE DYNAMIC vORTEx MotT TRANSITION a-c: Differential resistance $d V / d I$ vs. frustration parameter $f$ in the vicinity of $f_{c}=1 / 2,1,2$, respectively. Each panel contains 36 traces in $0.01 \mathrm{~mA}$ steps. The colors refer to magnitude of the current. The dots mark $(d V / d I)\left(I=I_{0}\right)$-s separating insulating-like and metallic-like behaviours. The separatrix traces are chosen so that $\mathrm{dV} / \mathrm{dI}$ start to turn upwards on approach to $f_{c}$ at I $>I_{0}, d V / d I$ maintain downward trend near $f_{c}$ at $I<I_{0}$, and $d(d V / d I) /\left.d f\right|_{f=f_{c}}=0$ at $\mathrm{I}=\mathrm{I}_{0}$. In the metallic regions $\mathrm{dV} / \mathrm{dI}$ shows minima marked with strokes which enclose the critical regions around $f_{c}$. The traces are not symmetric with respect to $f_{c}$ so the critical currents for left-, $I_{0}^{-}$, and right-hand side, $\mathrm{I}_{0}^{+}$, vicinities are different and assume the values a: $I_{0}^{-}=0.83 \mathrm{~mA}, f=1 / 2_{-} ; I_{0}^{+}=0.78 \mathrm{~mA}, f=1 / 2_{+}$; b: $I_{0}^{-}=1.10 \mathrm{~mA}, f=1_{-} ; I_{0}^{+}=1.14 \mathrm{~mA}, f=1_{+} ; \mathbf{c}:$ $I_{0}^{-}=0.45 \mathrm{~mA}, f=2_{-} ; I_{0}^{+}=0.48 \mathrm{~mA}, f=2_{+}$. d-f: Scaling of the same data after subtracting the corresponding separatrix', $d V / d I-(d V / d I)\left(I=I_{0}\right)$ with respect to variable $\left|\mathrm{I}-\mathrm{I}_{0}\right| /|\mathrm{h}|^{(\delta-1) / \delta}$ with $\mathrm{h}=\mathrm{f}-\mathrm{f}_{\mathrm{c}}$ and $\delta=2$ for $f_{c}=1 / 2(d)$ and $\delta=3$ for $f_{c}=1$ and $f_{c}=2(e, f)$. The lefthand side (right-hand side) subpanels present the scaling plots in the $f_{c}-|h|(+|h|)$ vicinities of the critical frustration parameters. Upper panels employ the linear scales of $d V / d I-(d V / d I)\left(I=I_{0}\right)$, whereas in the lower panels the logarithmic scales are used. The scales are the same for each $f_{c}$ pairwise for left-hand side and right-hand side subpanels, The black lines in the lower panels correspond to powers $\mu=1$ for $f_{c}=1 / 2$, and to $\mu=1.2 \pm 0.03$. 
istic tunnelling energy or bandwidth, J. Accordingly, the static Mott transition exhibits scaling behaviour with respect to temperature, $T$ and the repulsion strength $\mathrm{U}$. The mean-field version of the Hubbard model predicts [40] that near the Mott critical point $\left(T_{c}, U_{c}\right)$, where $T_{c}$ and $U_{c}$ are critical temperature and critical particle repulsion strength respectively, the deviation $\Delta \mathrm{U}=\left|\mathrm{U}-\mathrm{U}_{\mathrm{c}}\right|$ should scale as $\left(\mathrm{T}_{\mathrm{c}}-\mathrm{T}\right)^{\delta /(\delta-1)}$, where $\delta$ is the exponent relating the scaling of the source field with the order parameter. To figure out the critical behaviour of the dynamic vortex Mott transition, we note that the role of $U$ is taken by the magnetic field $B$ which sets the vortex density and, as such, controls the vortex repulsion. The corresponding critical field values are at commensurate $f_{c}=p / q$. Using a mean field approximation [21] we conjecture a mapping between temperature and bias current, with the critical temperature $T_{c}$ replaced by the critical current $\mathrm{I}_{0}$ separating distinct dynamic states. We thus posit the following mapping of the critical behaviour of the static Mott transition to that of dynamic vortex Mott transition: $\left|\mathrm{U}-\mathrm{U}_{\mathrm{c}}\right| \rightarrow \mathrm{h}=\left|\mathrm{f}_{\mathrm{c}}-\mathrm{f}\right|,\left|\mathrm{T}-\mathrm{T}_{\mathrm{c}}\right| \rightarrow\left|\mathrm{I}-\mathrm{I}_{0}\right|$, and the critical point $\left(\mathrm{T}_{\mathrm{c}}, \mathrm{U}_{\mathrm{c}}\right) \rightarrow\left(\mathrm{I}_{0}, \mathrm{f}_{\mathrm{c}}\right)$ yielding critical scalings $\left|\mathrm{I}-\mathrm{I}_{0}\right| \sim|\mathrm{h}|^{(\delta-1) / \delta}$.

To show the validity of this assumption, we scale our $d V / d I$ data into a universal form

$$
(d V / d I)(f, I)-(d V / d I)\left(f, I_{0}\right)=\mathcal{F}_{ \pm}\left(\frac{\left|I-I_{0}^{ \pm}\right|}{|h|^{(\delta-1) / \delta}}\right)
$$

taking the exponent $\delta$ as an adjustable fitting parameter. The best collapse of the data onto a single curve near $f_{c}=1 / 2$ is achieved at $\delta=2$ (Fig. $7 \cdot 4 \mathrm{~d}$ ). Importantly, the independent scaling procedures at both the left- and right-hand sides of the critical frustration yield the same value of $\delta$, despite the different $\mathrm{I}^{ \pm}$separatrices. The same procedure for the left- and right-hand sides of $f_{c}=1$ and 2 gives rise to $\delta=3$, see Fig. 7.4e,f. Doublelogarithmic plots in the vicinity of the above values of $f_{c}$ display a power-law functional form of $\mathcal{F}_{ \pm}(x) \propto \chi^{\mu}$, as shown in the lower panels of Fig. 7.4d-f. For $f_{c}=1 / 2$ we find $\mu=1 \pm 0.03$, and for $f_{c}=1,2$, we determine the same $\mu=1.2 \pm 0.03$ for all four plots. The revealed universal scaling properties of the current and magnetic field dependent differential resistance, following Eq.7.1, experimentally establishes the dynamic vortex Mott transition and the existence of the vortex Mott insulator in superconducting networks. 


\subsection{CONCLUSION}

To conclude we consider the implications of our results. Firstly, the energy spectrum of the fractional vortex Mott insulator shown in Fig.7.1c is the Hofstadter butterfly spectrum of the Harper equation [30]. The latter is also the spectrum of the fractional quantum Hall electronic state [33]. This correspondence between the fractional vortex Mott insulating- and fractional quantum Hall electronic states and offers a 'vortex-side' evidence for the charge-flux duality previously observed near the quantum Hall liquid to insulator transition [34].

Secondly, our value of $\delta=3$ for the integer dynamic vortex Mott transition notably coincides with the mean-field value of $\delta$ for the equilibrium, temperature-pressure driven Mott transition $[17,41]$. It reveals a deep correspondence between the dynamic and thermodynamic Mott transitions. It supports the conjecture $[21,42]$ that the external drive takes the role of effective temperature in far-from-equilibrium systems. Furthermore, our finding offers experimental evidence of the quantum mechanical vortex mapping establishing a duality between vortices and $2 \mathrm{D}$ quantum particles [22].

Finally, turning to the difference between critical exponents at integer and fractional frustration parameters $\left(\delta=3\right.$ for $f_{c}=1,2$ and $\delta=2$ for $f_{c}=1 / 2$ ) we review the difference in filling of the pinning sites at these commensurate fields. An integer vortex Mott insulator corresponds to full filling of all the sites with vortices, and can be viewed as a 'ferromagnetic' state. On the other hand the case of $f=1 / 2$ represents an 'antiferromagnetic' state since the direction of currents in "empty" cells corresponds to vortices of the opposite direction. Therefore, the critical behaviour of these two distinct vortex Mott insulating phases expectedly belongs in different universality classes with correspondingly different exponents. In arrays with more profound dips at further $f_{c}$ the phase transition of the corresponding fractional vortex Mott states and their respective critical exponents will likely reveal more insights into Mott physics in general. The gained insights open a route to in-depth investigation of dynamic phase transitions in strongly correlated fermionic and bosonic systems. 
[1] N. F. Mott and R. Peierls, Proc. Phys. Soc. 49 (4S), 72-73 (1937).

[2] N.F. Mott , Proc. Phys. Soc. A 62, 416-422 (1949).

[3] N. F. Mott, Metal-Insulator Transitions. (Taylor and Francis, London, 1990).

[4] M. Imada, A. Fujimori, and Y. Tokura, Rev. Mod. Phys. 70, 1039-1263 (1998).

[5] P. A. Lee, N. Nagaosa, and X.-G. Wen, Rev. Mod. Phys. 78, 17-85 (2006).

[6] D. Jaksch, C. Bruder, J. I. Cirac, C. W. Gardiner and P. Zoller, Phys. Rev. Lett. 81, 3108-3111 (1998).

[7] W. Hofstetter, J. I. Cirac, P. Zoller, E. Demler and M.D. Lukin, Phys. Rev. Lett. 89, 220407 (2002).

[8] M. Greiner, O. Mandel, T. Esslinger, T.W. Hänsch and I. Bloch, Nature, 415, 39-44 (2002).

[9] I. Bloch, J. Dalibard and W. Zwerger, Rev. Mod. Phys. 8o, 885-964 (2008).

[10] R. Jördens, N. Strohmaier, K. Günter, H. Moritz and T. Esslinger, Nature 455, 204-207 (2008).

[11] J. F. Sherson, C. Weitenberg, M. Endres, M. Cheneau, I. Bloch and S. Kuhr, Nature 467, 68-72 (2010).

[12] C. Weitenberg, M. Endres, J. F. Sherson, M. Cheneau, P. Schauß, T. Fukuhara, I. Bloch and S. Kuhr, Nature 471, 319$324(2011)$.

[13] D. Watanabe, M. Yamashita, S. Tonegawa, Y. Oshima, H. M. Yamamoto, R. Kato, I. Sheikin, K. Behnia, T. Terashima, S. Uji, T. Shibauchi and Y. Matsuda, Nature Communications, 3, 1090 (2012).

[14] Y. Taguchi, T. Matsumoto and Y. Tokura, Phys. Rev. B62, 7015-7018 (2000).

[15] V. Guiot, L. Cario1, E. Janod, B. Corraze, V. Ta Phuoc, M. Rozenberg, P. Stoliar, T. Cren and D. Roditchev Nature Comm, 4, 1722 (2013) 
[16] M. Eckstein, T. Oka and P. Werner Phys. Rev. Lett. 105, 146404 (2010).

[17] P. Limelette, A. Georges, D. Jérome, P. Wzietek, P. Metcalf and J. M. Honig Science 302, 89-92 (2003).

[18] M. Nakano, K. Shibuya1, D. Okuyama1, T. Hatano1, S. Ono, M. Kawasaki, Y. Iwasa and Y. Tokura Nature, 487, 459462 (2012).

[19] T. Oka and H. Aoki, Phys. Rev. B 81, 033103 (2010).

[20] F. Heidrich-Meisner, I. Gonzalez, K. A. Al-Hassanieh, A. E. Feiguin, M.J. Rozenberg and E. Dagotto Phys. Rev. B82, 20511 (2010).

[21] N. Chtchelkatchev and V. M. Vinokur Europhys. Lett., 88, 47001 (2009).

[22] D. R. Nelson and V.M. Vinokur, Phys. Rev. B 48, 1306013097 (1993).

[23] R. S. Newrock, C. J. Lobb, U. Geigenmüller and M. Octavio Solid State Phys. 54, 263-512 (2000).

[24] V. V. Deshpande, B. Chandra, R. Caldwell, D. S. Novikov, J. Hone and M. Bockrath, Science 323, 106-110 (2009).

[25] In an electronic Mott insulator the density of states is probed by the differential conductivity, dI/dV [24]. In the insulating state the density of states has a dip at zero bias. Upon the transition to a metallic state it turns into a peak. In a vortex system, according to the quantum mechanical mapping correspondence [22], the differential conductance is substituted by the differential resistance.

[26] S. Eley, S. Gopalakrishnan, P. M. Goldbart and N. Mason, Nature Phys. 8, 59-62 (2012).

[27] V. L. Berezinskii, Sov. Phys. JETP 32 (3): 493-500 (1971)

[28] J.M. Kosterlitz and D. J. Thouless, Journal of Physics C: Solid State Physics 6, 1181-1203 (1973)

[29] D. J. Resnick, J. C. Garland, J. T. Boyd, S. Shoemaker and R.S. Newrock, Phys. Rev. Lett. 471542 (1981)

[30] D. R. Hofstadter, Phys. Rev. B 14, 2239-2249 (1976). 
[31] P. G. Harper, Proc. Phys. Soc. A 68 874-878 (1955).

[32] T.I. Baturina, V.M. Vinokur, A. Yu. Mironov, N.M. Chtchelkatchev, D. A. Nasimov and A.V. Latyshev, Europhys. Lett. 93, 47002 (2011).

[33] C.R. Dean, L. Wang, P. Maher, C. Forsythe, F. Ghahari, Y. Gao, J. Katoch, M. Ishigami, P. Moon, M. Koshino, T. Taniguchi, K. Watanabe, K. L. Shepard, J. Hone and P. Kim, Nature 497, 598-602 (2013).

[34] D. Shahar, D. C. Tsui, M. Shayegan, E. Shimshoni and S. L. Sondhi, Science 274, 589-592 (1996).

[35] S.P. Benz, M.S. Rzchowski, M. Tinkham, and C.J. Lobb, Phys. Rev. B 42, 6165-6171 (1990).

[36] Flips of the dips in $d V / d I$ at commensurate fillings $f<1$ upon increasing current in a proximity array has been noticed in [35] and attempted to explain by depinning of vortices at currents exceeding the corresponding depinning current. This, however, should have resulted also in the peak of the resistance and not only in $\mathrm{dV} / \mathrm{dI}$.

[37] S. L. Sondhi, S. M. Girvin, J. P. Carini and D. Shahar, Rev. Mod. Phys. 69, 315-333 (1997).

[38] L.P. Kadanoff, W. Götze, D. Hamblen, R. Hecht,E. A.S. Lewis, V. V. Palciauskas, M. Rayl, J. Swift, D. Aspnes and J. Kane, Rev. Mod. Phys. 39, 395-431 (1967).

[39] M.E. Fisher Rev. Mod. Phys. 46, 597-616 (1974).

[40] G. Kotliar, E. Lange and M. J. Rozenberg, Phys. Rev. Lett. 84, 5180-5183 (2000).

[41] In the equilibrium case the critical behavior is demonstrated in [17] by scaling of the conductivity as function of temperature $T$ and the applied pressure $P$ (serving to change the electron density) in a form $\sigma(\mathrm{P}, \mathrm{T})-\sigma_{\mathrm{c}}=$ $(\delta h)^{1 / 3} f_{ \pm}\left(\delta h / r^{3 / 2}\right)$ (the 'effective field' $\delta h=\left(P-P_{I}\right) / P_{c}, P_{I}$ and $P_{c}$ were spinodal and critical pressures, respectively, and $\left.\mathrm{r}=\left|\mathrm{T}-\mathrm{T}_{\mathcal{c}}\right| / \mathrm{T}_{\mathcal{c}}\right)$.

[42] A. E. Koshelev and V. M. Vinokur, Phys. Rev. Lett. 73, 35803583 (1994). 

In Josephson Junction arrays composed of $\mathrm{Nb}$ superconducting islands on a Au surface we investigate the effect of $\mathrm{Nb}$ island removal. The removal of islands reveals that there is appreciable coupling between next-nearest neighbours, which reduces the percolation threshold from 0.59 to 0.45 . Island removal destroys the translational symmetry of the system, a prerequisite for using the Hofstadter approach for explaining the structure in the magnetoresistance at fields commensurate with the physical lattice. However, in our samples structure remains visible in magnetoresistance traces for arrays with up to $60 \%$ of all islands removed. This is attributed to the existence of $n \times n$ islands plaquettes in these arrays.

\subsection{BACKGROUND}

The behaviour of arrays of Josephson junctions at low temperatures $[1,2]$ and zero field is dominated by a BerezinskiiKosterlitz-Thouless phase transition [3-5], when a quasi long range ordering of the phases of the superconducting islands develops. Excitations of the array take the form of vortices threading the array and distorting the phases on the islands. Below a threshold temperature $\mathrm{T}_{\mathrm{BKT}}$, excitations are only feasible as vortex-anti-vortex pairs. Above this temperature free single vortices are also present. Applying a magnetic field creates a majority vortex population. The behaviour in a magnetic field reveals a rich structure: where a single superconducting loop or dcSQUID show oscillations in $\Phi_{0}$ in resistance, supercurrent and transition temperature $[6,7]$, an array exhibits oscillations with commensurate fields, or when a rational fraction of flux quanta threads a unit area [8-11]. The behaviour of this system can be described using the Harper equation [12, 13], which is a one dimensional reduction of the Hofstadter problem [14].

One of the simplest situations to visualise in such a system is when half a flux quantum threads a unit area set by the lattice 

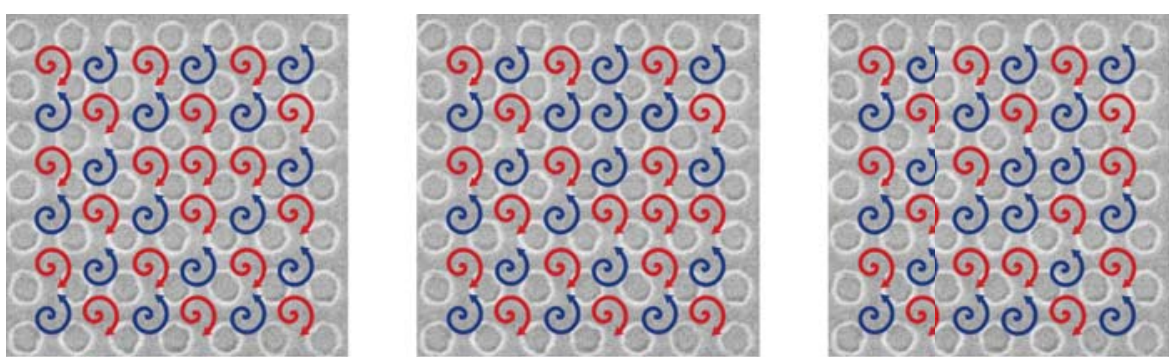

Figure 8.1: Excitations IN A FULLY FRUSTRATED ARRAy. The left and middle panel illustrate an isolated vortex and a vortexantivortex pair disturbing the checkerboard pattern. The right panel shows two competing checkerboard patterns.

spacing a. This is also known as a fully frustrated array. In this situation, a checkerboard pattern of vortices will form in the array $[5,8]$, resulting in a checkerboard of anti-vortices as well. The system has gained the ordering associated with an antiferromagnetic system. Several excitations can occur in such a system, shown in figure 8.1. An isolated vortex (left panel) or a vortex-antivortex pair (middle panel) are associated with a BKT system. However, due to the presence of a double degeneracy in the ground state (the whole checkerboard pattern can translate by a distance a) at finite temperatures both patterns may exist (right panel). These are excitations associated with an Ising system.

In an Ising system one can look at the influence of site disorder: removing spins from the $2 \mathrm{D}$ system such as in a dilute (anti)ferromagnet. Larger clusters can show local order without the presence of global order throughout the system. Only when the coupling between clusters is sufficiently large will there be global order [15]. The behaviour of high temperature superconductors may be placed in this light. One of the ideas to explain the existence of a pseudogap phase are disconnected clusters showing individual ordering without global phase coherence [16]. Only below the critical temperature is there global phase coherence, and above this temperature individual clusters are responsible for the pseudogap. It has been proposed that the disorder in these systems is actually the key to understanding the behaviour of high temperature superconductors: at the percolative threshold there may be an enhancement of the critical temperature [17]. The percolation threshold here ensures that (nearly) all clusters are still connected, while the length scale associated with the hole cluster size, the areas without islands, diverges. 


\subsection{SUPERCONDUCTING TRANSITION TEMPERATURE*}

To directly test a possible enhancement in the transition temperature around the percolation threshold, the resistance of arrays consisting of $300 \times 300$ island positions with filling factors $p=1,0.75,0.70, \ldots, 0.4$ are measured as a function of temperature and illustrated in figure 8.2. Island positions are filled with $\mathrm{Nb}$ islands of $45 \mathrm{~nm}$ thick, $109 \mathrm{~nm}$ diameter and $44 \mathrm{~nm}$ spacing on a $40 \mathrm{~nm}$ thick Au substrate. Fabrication and measurement methods are identical to the ones used in chapter $7^{1}$. The patterns with the percolating arrays were provided by Bianconi [17] and are based on a scale free network.

It is clear from figure 8.2 that there is no enhancement of the transition temperature with reduced filling factor $p$. Instead, a reduction in the transition temperature and increase in transition width is evident. First, we look at the behaviour of the transition width and transition temperature for a completely filled array. In this case, the array undergoes a BKT transition, below which there are no individual excitations or vortices in the system. The transition temperature is given by:

$$
\mathrm{T}_{\mathrm{BKT}}=\frac{\pi \mathrm{E}_{\mathrm{J}}}{2 \mathrm{k}_{\mathrm{B}}},
$$

where $E_{J}$ is the Josephson coupling between two neighbouring islands. This can be well below the transition temperature of the individual islands resulting in a widely smeared transition $[2,18,19]$. For site reduced arrays there is a similar reduction and broadening $[20,21]$, and the filling factor $p$ dependent transition temperature is given by:

$$
\frac{\mathrm{T}_{\mathrm{BKT}}(\mathrm{p})}{\mathrm{T}_{\mathrm{BKT}}(1)} \sim\left(\mathrm{p}-\mathrm{p}_{\mathrm{c}}\right)^{\mathrm{t}},
$$

where $t=1.30$ and $p_{c}$ is the critical percolation fraction. For direct neighbour site percolation $p_{c} \approx 0.59$. For percolating systems holes exist in the lattice where unbound vortices can exist to lower temperatures, reducing the transition temperature. Below $p_{c}$ the holes are larger than the device dimensions and flux can penetrate the sample freely. Cooling down to $2 \mathrm{~K}$ only the devices with $p \geqslant 0.6$ show a transition to a zero resistance state, but the devices with $p<0.6$ can be expected to have a complete transition as well at even lower temperatures. On a tightly

\footnotetext{
* Sample preparation by Nicola Poccia and Francesco Coneri.

${ }^{1}$ The devices with filling factors between 1 and 0.55 were fabricated in one run, the other three filling factors were part of a different run.
} 


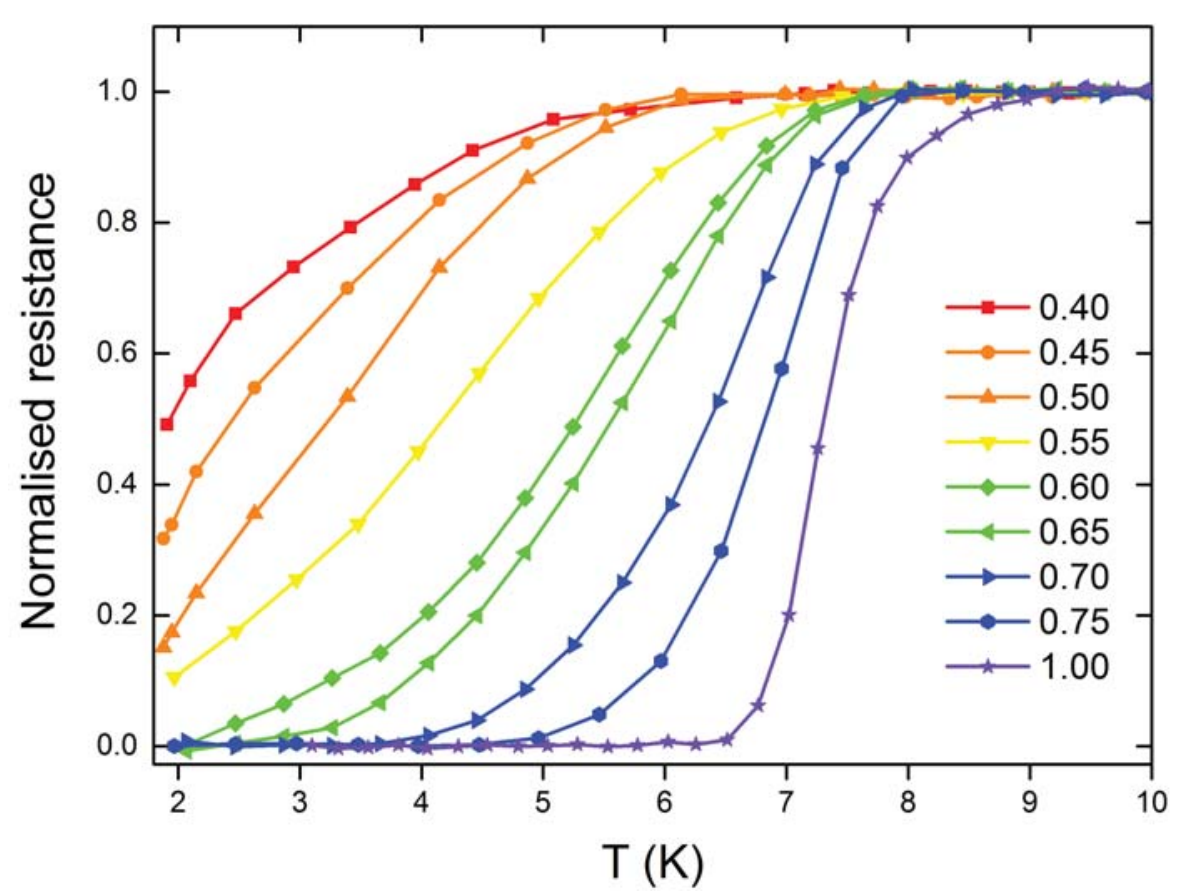

Figure 8.2: TRANSITION TEMPERATURE AS FUNCTION OF FILLING FACTOR $p$. From left to right, the various curves span increasing filling factors, from $p=0.4$ to $p=0.75$, with steps $\Delta p=0.05$. The curve for the regular pattern is also presented, with strong coupling between islands. For $p \geqslant 0.6$ the superconducting state sets in in the measured temperature range, with an onset temperature that increases with filling factor. A bias current of $5 \mu \mathrm{A}$ was applied while warming up from the base temperature of $1.9 \mathrm{~K}$, at a sweep rate of $0.3 \mathrm{~K} / \mathrm{sec}$. The curves are normalised to the $\mathrm{R}(1 \mathrm{OK})$ value. 
coupled island network without sharply defined junctions, as is the case in our devices, it is reasonable to assume that next nearest neighbour interaction plays a role as well. The supercurrent and Josephson coupling in a SNS junction decreases exponentially with distance [22] when the electrode separation exceeds the coherence length. Therefore there is a non-negligible coupling between diagonal neighbours and even between further removed neighbours. This reduces the percolation threshold $p_{c}$.

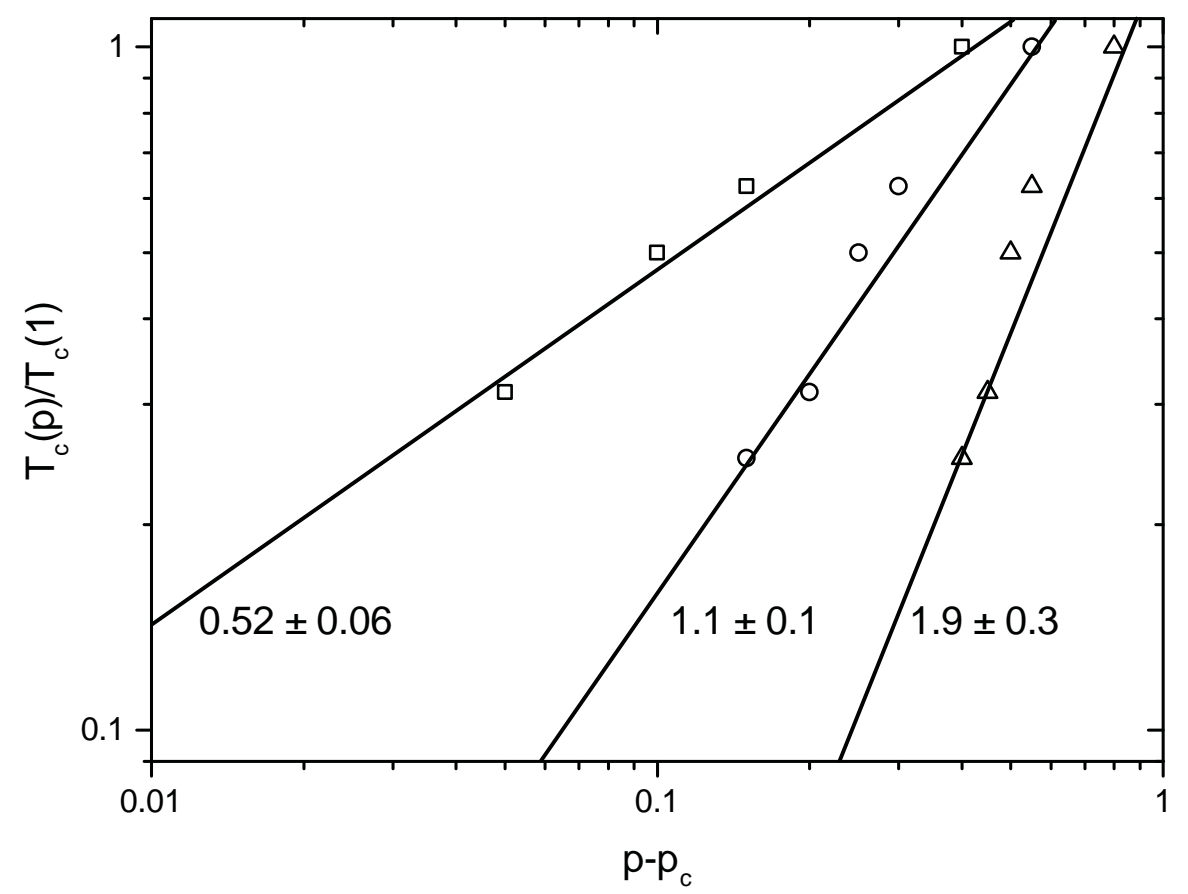

Figure 8.3: REDUCED TRANSITION TEMPERATURE AS A FUNCTION OF REDUCED DISORDER. The square, circle and triangle symbols are rescaled for different percolation thresholds $p_{\mathrm{c}}=$ $0.6,0.45,0.2$ respectively, determined by taking into account coupling between islands within a distance radius of $a, \sqrt{2} a$ and $\sqrt{8} a$. The solid lines are fits to equation 8.2 and the corresponding slopes/exponent $t$ is indicated as well. The expected value of $t$ is 1.3 [23].

The percolation threshold as a function of nearest neighbour coupling can be estimated using a universal formula [24, 25]. For nearest neighbours within a radius of $a, \sqrt{2} a$ and $\sqrt{8} a$ the percolation thresholds are $0.59,0.28$ and 0.1 respectively. For the devices used the percolation threshold is determined by solving the maximum flow problem for a graph of connected vertices (islands) with flow between vertices exponentially decreasing with separation and cut off at varying radii. The maximum flow is dual to the minimum cut of a graph, which gives 

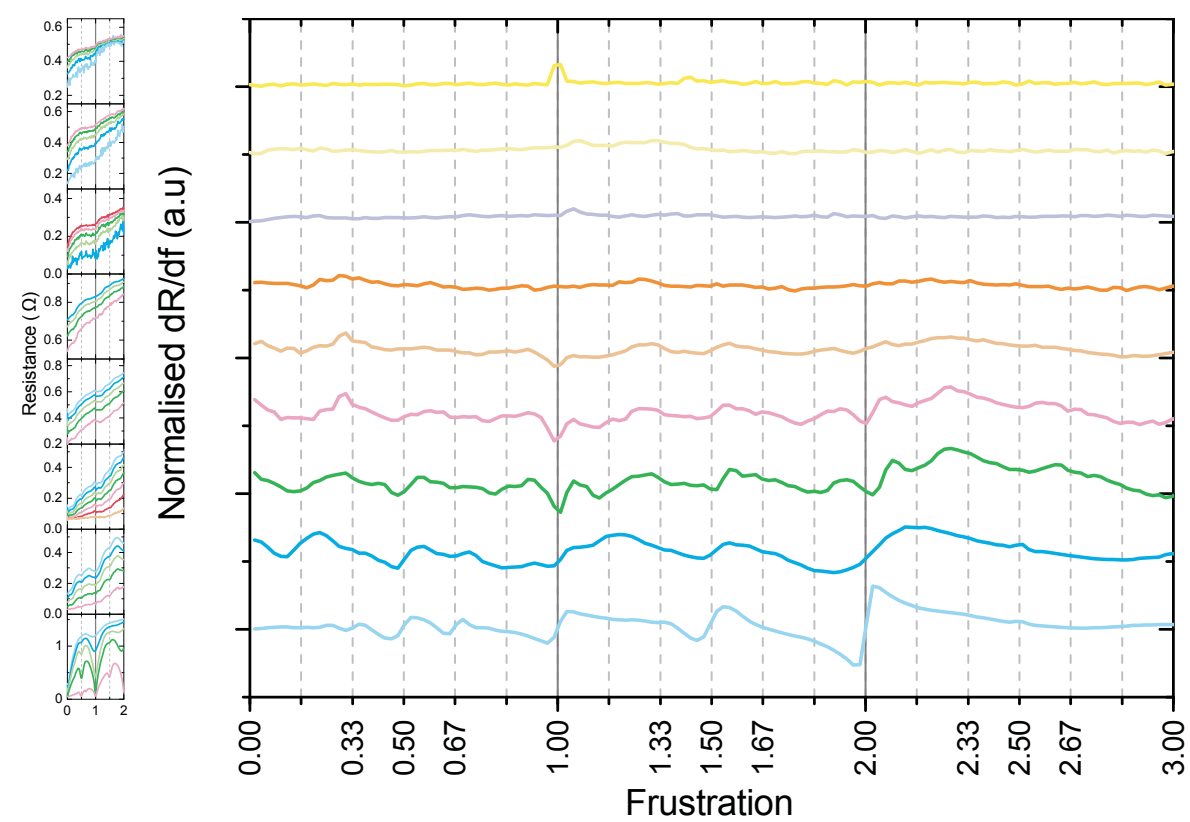

Figure 8.4: Resistance OsCillations. The left graph shows normalised resistance traces for the different arrays as function of frustration $f$. The completely filled array clearly shows a richer structure. The right graph shows the derivative $d R / d f$ as a function of $f$. Here, the persistence of the richer structure for the completely filled array into diluted arrays is clear. In both cases, from bottom to top, the filling factor $p$ increases: $1,0.75,0.70, \ldots, 0.4$.

the minimal number of removed connections to completely disconnect two points in a graph. In our case we find maximum flow between a point connected to all islands on the left of the array to a point connected to all islands on the right, to account for bus bars on the samples. These superconducting bus bars ensure a homogeneous current injection in the array. For the radii mentioned previously the thresholds are 0.6, 0.45 and 0.2. These thresholds have been used to plot the reduced transition temperature versus reduced sample disorder in figure 8.3.

The solid lines are fits using equation 8.2 using the different percolation thresholds. When including diagonally connected neighbours as well, or islands within $\sqrt{2} a$, the slope, $1.1 \pm 0.1$, approaches the expected value of 1.3. Deviations in fitting are expected due to finite sample dimensions [5] as well as uncertainty in determining the exact transition temperature. 


\subsection{BEHAVIOUR IN MAGNETIC FIELD}

We now turn to the behaviour of percolating arrays in magnetic fields to investigate if such systems show collective behaviour. An applied magnetic field increases the density of unpaired vortices in the system, $n_{f}=B / \Phi_{0}$. Flux can only penetrate a percolating system as flux quanta and thus frustrates the phases on the superconducting islands. Flux quanta repel each other and will arrange to minimise their potential energy, similar to Abrikosov lattices [26] in type-II superconductors, but with a physical lattice now limiting the positions they can inhabit.

Translational symmetry is broken in a percolating lattice. Referring back to the Hofstadter butterfly, the ansatz of a Bloch wave can no longer be made. The holes in the lattice provide lower energy positions for quanta to inhabit. In chapter 7 the fully filled device was investigated and revealed oscillations at rational frustration $f=p / q$. In figure 8.4 resistance and $d R / d f$ traces are presented for reduced filling factor. The peculiar behaviour of $\mathrm{dV} / \mathrm{dI}$ for increasing current at a fixed frustration, the subject of the previous chapter is not present in any reduced array.

The disappearance of rich structure at rational frustration is understandable as a consequence of the disappearance of translational symmetry. However, the persistence of the structure at integer frustration is understandable when considering an empty array save for one plaquette of four islands connected in a square. This $2 \times 2$ plaquette is subject to flux quantisation in the same way as a superconducting ring $[27,28]$ and subject to oscillations in the transition temperature with the applied field [6]. Even in a device below the percolation threshold the existence of such a structure will result in a periodic structure. In devices above the percolation threshold but lacking translational symmetry the system can be visualised as a collection of weakly coupled plaquettes. This is the situation which we find in our devices: despite the absence of translational symmetry we observe oscillations with commensurate fields.

The existence of larger plaquettes, $n \times n$ islands, allows construction of solutions for frustration $f=p /(n-1)$. This should result in structure in the magnetoresistance at these matching fields. For all disordered arrays down to $p=0.55$, structure is still present in $d R / d f$ traces at $f=1 / 3,1 / 2,2 / 3$. Despite the loss of global translational symmetry, the isolated plaquettes of 

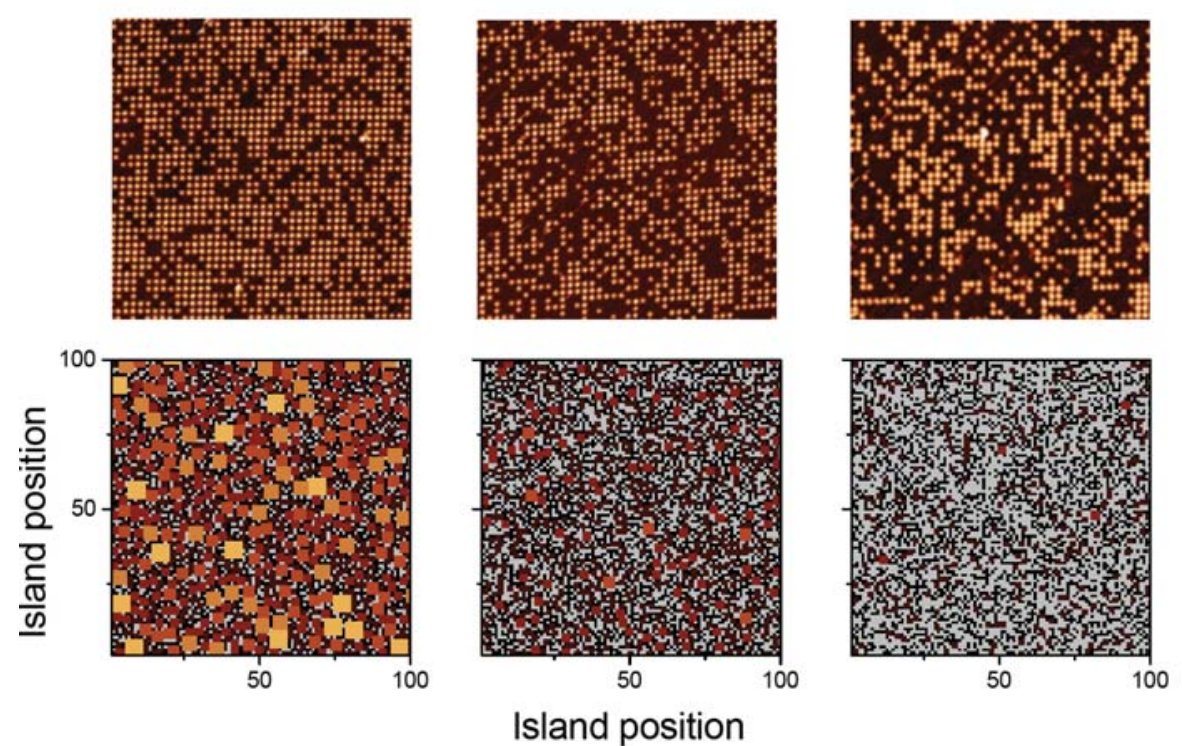

Figure 8.5: Visualisation of CLUSTER Sizes. The top row images are $10 \mu \mathrm{m} \times 10 \mu \mathrm{m}$ AFM images of arrays with $p=$ $0.75,0.55,0.4$. The light dots are $\mathrm{Nb}$ islands. The bottom row shows a visualisation of square cluster sizes, where clusters of similar dimensions are labelled with a colour. Empty positions are light grey. The top and bottom images do not correspond to the same area.

up to $4 \times 4$ present even in extremely diluted arrays may still provide structure in the resistance traces.

\subsection{CLUSTER SIZES IN RANDOM ARRAYS}

We attribute the persistence of oscillations at $f=1 / 3,1 / 2,2 / 3$, even in diluted arrays, to the existence of plaquettes of size $3 \times 3$ and $4 \times 4$ down to $p=0.4$. In the top panels of figure 8.5 AFM images of arrays with $p=0.75,0.55,0.4$ are visible, along with colour coded representations of the same filling factors in the bottom panels. In figure 8.6 the average cluster size and the cluster size distribution as function of filling factor are plotted. Included are also representations of higher and lower filling factors which were not measured.

For $p=0.75$ more than $62 \%$ of the islands are part of a square structure. For the lowest filling measured, $p=0.4$, this has fallen to $18 \%$, and there are $273 \times 3$ clusters present in an array of $300 \times 300$ islands. Only below $p=0.25$ do these clusters disappear. These $3 \times 3$ should result in oscillations at $f=1 / 2$. The clusters of size $4 \times 4$ only occur in arrays with $p>0.45$ : a 

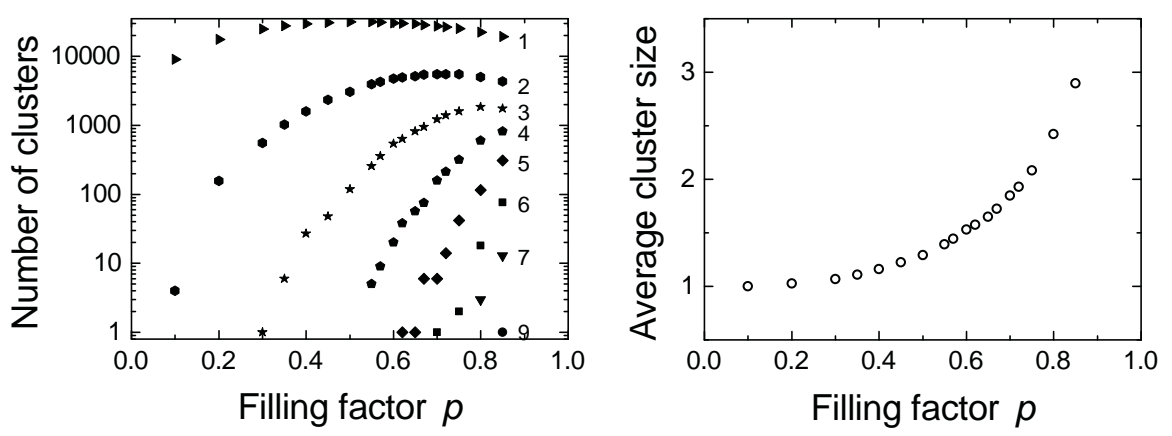

Figure 8.6: Cluster size Distribution. The left image shows the cluster size distribution as a function of array filling. As the filling factor decreases the maximum cluster size decreases, and initially the number of small clusters increases. As the array is diluted the number of smaller clusters also decreases. The right image shows the average cluster size: arrays with low filling are predominantly individual islands or part of a collection without any loops.

feature with the corresponding period is visible for $p \geqslant 0.55$ in transport measurements.

\subsection{CONCLUSION}

A scaling analysis of the zero voltage state as a function of temperature reveals that the percolation threshold in $\mathrm{Nb}$ islands / Au substrate arrays is well below the expected value of $p_{c} \sim 0.6$. Instead, a percolation threshold $p_{c}=0.45$ is found, indicating appreciable coupling to next-nearest, or diagonal, neighbours as well. This differentiates the investigated devices from previous work on Josephson junction arrays [5, 29].

When reducing the filling factor of devices the rich structure due to commensurability diminishes. However, the oscillations persist in all filling factors investigated, down to $p=0.4$. We attribute this to the existence of plaquettes of $2 \times 2,3 \times 3$ and $4 \times 4$ islands at these reduced filling factors, which in turn are responsible for oscillations at $f=1,1 / 2,1 / 3$ and multiples.

The existence of larger plaquettes weakly connected by single links to each other was proposed to enhance the critical temperature of the complete device. No such enhancement occurs in our devices. Instead, these plaquettes can be regarded individually; this explains the magnetoresistance behaviour of diluted arrays. They do not have to be connected to each other via a superconducting link. Rather, they form areas of zero re- 
sistance on a resistive background, and currents redistribute to minimise the total resistance. The modulation of the supercurrent with external current is reflected in a field modulated resistance.

\section{BIBLIOGRAPHY}

[1] D. Resnick, J. Garland, J. Boyd, S. Shoemaker, and R. Newrock, Phys. Rev. Lett. 47, 1542 (1981).

[2] D. W. Abraham, C. J. Lobb, M. Tinkham, and T. M. Klapwijk, Phys. Rev. B 26, 5268 (1982).

[3] V. Berezinskii, Sov. Phys. JETP 32 (3), 493 (1971).

[4] J. Kosterlitz and D. Thouless, Journal of Physics C: Solid State Physics 6, 1181 (1973).

[5] R. Newrock, C. Lobb, U. Geigenmüller, and M. Octavio, Solid State Physics 54, 263 (2000).

[6] W. A. Little and R. D. Parks, Phys. Rev. Lett. 9, 9 (1962).

[7] R. C. Jaklevic, J. Lambe, A. H. Silver, and J. E. Mercereau, Phys. Rev. Lett. 12, 159 (1964).

[8] M. Tinkham, D. Abraham, and C. Lobb, Phys. Rev. B 28, 6578 (1983).

[9] R. A. Webb, R. F. Voss, G. Grinstein, and P. M. Horn, Phys. Rev. Lett. 51, 690 (1983).

[10] S. Teitel and C. Jayaprakash, Phys. Rev. B 27, 598 (1983).

[11] B. Pannetier, J. Chaussy, R. Rammal, and J. C. Villegier, Phys. Rev. Lett. 53, 1845 (1984).

[12] P. G. Harper, Proceedings of the Physical Society. Section A 68, 874 (1955).

[13] T. I. Baturina, V. M. Vinokur, A. Y. Mironov, N. M. Chtchelkatchev, D. A. Nasimov, and A. V. Latyshev, EPL (Europhysics Letters) 93, 47002 (2011).

[14] D. R. Hofstadter, Phys. Rev. B 14, 2239 (1976).

[15] R. B. Griffiths, Phys. Rev. Lett. 23, 17 (1969). 
[16] M. Buchanan, Nature 409, 8 (2001).

[17] G. Bianconi, Journal of Statistical Mechanics: Theory and Experiment 2012, Po7021 (2012).

[18] C. J. Lobb, D. W. Abraham, and M. Tinkham, Phys. Rev. B 27, $150(1983)$.

[19] S. Eley, S. Gopalakrishnan, P. M. Goldbart, and N. Mason, Nat Phys 8, 59 (2012).

[20] D. C. Harris, S. T. Herbert, D. Stroud, and J. C. Garland, Phys. Rev. Lett. 67, 3606 (1991).

[21] I.-C. Baek, Y.-J. Yun, J.-I. Lee, and M.-Y. Choi, Phys. Rev. B 72, 144507 (2005).

[22] P. G. de Gennes, Rev. Mod. Phys. 36, 225 (1964).

[23] D. J. Frank and C. J. Lobb, Phys. Rev. B 37, 302 (1988).

[24] S. Galam and A. Mauger, Phys. Rev. E 53, 2177 (1996).

[25] K. Malarz and S. Galam, Phys. Rev. E 71, 016125 (2005).

[26] A. Abrikosov, Journal of Physics and Chemistry of Solids 2, 199 (1957).

[27] B. S. Deaver and W. M. Fairbank, Phys. Rev. Lett. 7, 43 (1961).

[28] R. Doll and M. Näbauer, Phys. Rev. Lett. 7, 51 (1961).

[29] P. Martinoli and C. Leemann, Journal of Low Temperature Physics 118, 699 (2000). 

This thesis explores two central themes: Josephson junctions on topological insulators and Josephson junction arrays. The promise of Majorana zero modes in topological insulator / superconductor systems is discussed in the first chapters of this thesis. A Majorana zero mode will potentially facilitate the realisation of quantum bits less sensitive to decoherence, reducing the number of error correction techniques necessary for the construction of a quantum computer. Before this stage can be reached the unambiguous detection and manipulation of these modes is necessary. In this thesis an experimental approach is pursued towards this goal. The emphasis on the methodological side is not on assuming the influence of Majorana zero modes, or a non-trivial topology, on the measured results, but by analysing the geometrical aspects of the experiments.

The second theme explored is the phases present in frustrated Josephson junction arrays. Here, the Josephson junction array serves as a model system for a Mott insulator, and the the frustration via an external magnetic field and bias current act as parameters to manipulate the phase diagram of a Mott system.

Concerning the first theme, a possible path towards Majorana zero modes is by combining bismuth based topological insulators with superconductors. To start, the progress in this field is reviewed. Topologically non-trivial surface states in materials such as $\mathrm{Bi}_{2} \mathrm{Te}_{3}, \mathrm{Bi}_{2} \mathrm{Se}_{3}$ and combinations of the two along with antimony were demonstrated using techniques such as ARPES. The realised Josephson junctions on these materials so far exhibit small characteristic energies $I_{C} R_{N}$ due to a non-superconducting channel. The non-insulating bulk of the topological insulators used in experiments acts as a shunt. The supercurrent is mediated by the surface connecting the superconducting electrodes. This strong coupling to the surface is a result of the anisotropic band structure in this class of materials. The current-phase relationship of the junctions has so far not demonstrated the contribution of a Majorana zero mode. Deviations in the magnetic interference or Fraunhofer pattern in several experiments are understood in the context of flux focussing, defects, and the geometry of the junctions on the surface of 
the topological insulator. A more careful investigation can be done by including Josephson junctions in loop structures. This allows for a better discrimination between intrinsic and extrinsic effects contributing to the current-phase relationship.

Keeping the electrode separation constant while varying the electrode width leads to varying aspect ratio Josephson junctions. This approach results in experimentally disentangling the shunt due to the conducting bulk and flux focussing in $\mathrm{Nb} / \mathrm{Bi}_{2} \mathrm{Te}_{3}$ Josephson junctions. This geometric consideration is sufficient for understanding the behaviour in magnetic field and the evolution of the $I_{C} R_{N}$ product as function of width. This result is in line with the estimated number of superconducting channels within the junction. A possible Majorana zero mode is not expected to be dominant compared to the order of hundred trivial modes in the junction.

To tackle the previous challenge of a conducting bulk, Josephson junctions were fabricated using a different topological insulator, $\mathrm{Bi}_{1.5} \mathrm{Sb}_{0.5} \mathrm{Te}_{1.7} \mathrm{Se}_{1.3}$. This material exhibits an insulating bulk, in contrast to $\mathrm{Bi}_{2} \mathrm{Te}_{3}$. However, the mean free path of the surface states is reduced in this material. The Josephson nature of the junctions, with a critical current of the order of IonA, is demonstrated by measuring the Fraunhofer pattern at $30 \mathrm{mK}$. The Fraunhofer pattern, with strongly suppressed side-lobes and periodicity of several $\Phi_{0}$, is explained using diffusive transport theory in a junction with open edges.

As previously mentioned, embedding Josephson junctions with a possible non-trivial character in a loop structure, also called a SQUID, allows for the determination of the currentphase relationship of the junctions. Using $\mathrm{Bi}_{2} \mathrm{Te}_{3}$ as the barrier material, the trivial nature of the Josephson junctions is demonstrated. The oscillations are $\Phi_{0}$ periodic, and the reduced modulation depth of the critical current is in line with the inductance of the loop. Models of different loop structures show that a $\Phi_{0}$ periodicity of a SQUID is not sufficient to determine that the junctions are trivial. Instead, the modulation depth of the SQUID needs to be analysed. A modulation depth reduced below the depth predicted by a large $\beta_{\mathrm{L}}$ is an indication of a $\Phi_{0} / 2$ current-phase relationship of the junctions. The amount of reduction is a measure of the ratio between $\Phi_{0} / 2$ and $\Phi_{0}$ periodic channels.

The second theme of this thesis is the behaviour of Josephson junction arrays in a magnetic field. These arrays, consisting of $300 \times 300$ niobium islands of $220 \mathrm{~nm}$ diameter and $45 \mathrm{~nm}$ thick- 
ness, are grown in a square lattice with a period of $267 \mathrm{~nm}$ on a gold substrate. When cooled below the transition temperature of the islands, the Josephson coupling between islands results in a egg-crate potential landscape for the excitations of this system, vortices. An applied magnetic field controls the number of vortices in the array, while an applied current results in a force acting on the vortices trapped in the array. For fields where there is integer frustration, or an integer number of vortices per egg-crate dimple, a phase transition as a function of applied current is observed. For half-integer frustration this phase transition occurs with different critical exponents. An appropriate mapping exists between this vortex-based Mott insulator and an electronic Mott insulator, translating the current and field to the potential and doping.

By removing niobium islands from the array, the egg-crate potential is disturbed. Translational symmetry is necessary for constructing solutions describing such systems. However, the existence of small clusters provides enough symmetry on a local scale to show oscillations reminiscent of an undisturbed array. The percolation threshold, determined by the relationship between the transition temperature and the fraction of removed islands, is lower than expected when only considering nearest neighbour coupling. This is due to Josephson coupling between islands not being directed only between nearest neighbours. Removing islands reveals a sizeable coupling between next nearest neighbours as well. 

In dit proefschrift komen twee thema's aan bod: Josephson juncties op topologische isolatoren en roosters van Josephson juncties. In de eerste hoofdstukken van dit proefschrift worden de zogenaamde Majorana nulmodi in systemen van topologische isolatoren en supergeleiders besproken. Een Majorana nulmodus kan een grote rol spelen in de realisatie van een kwantumbit die minder gevoelig is voor decoherentie, waardoor minder foutcorrectietechnieken nodig zijn om een kwantumcomputer te bouwen. Voordat men hier aan toe is, moet de aanwezigheid en beheersbaarheid van deze modi buiten kijf staan. In dit proefschrift wordt een experimenteel pad afgelegd richting dat doel. De nadruk ligt bij het verklaren van resultaten via geometrische beschouwingen, zonder de aanwezigheid van een niet-triviale topologie of een Majorana nulmodus.

Het tweede thema is het gedrag van verschillende fases in roosters van Josephson juncties. Een dergelijk rooster is een modelsysteem voor een Mott-isolator, waarbij de frustratie via een extern magneetveld en een aangelegde stroom onderzoek van het fase-diagram van een Mott-systeem mogelijk maken.

Voor het eerste thema is een mogelijke route naar een Majorana nulmodus één via een combinatie van op bismut gebaseerde topologische isolatoren en supergeleiders. Als eerste zijn de ontwikkelingen op dit gebied samengevat. De vereiste topologisch niet-triviale oppervlakte toestanden is reeds aangetoond met technieken zoals ARPES in materialen als $\mathrm{Bi}_{2} \mathrm{Te}_{3^{\prime}}$, $\mathrm{Bi}_{2} \mathrm{Se}_{3}$ en combinaties van die twee. Wat opvalt bij Josephson juncties op deze materialen is dat de niet-isolerende bulk in de gebruikte topologische isolatoren zorgt voor een lage karakteristieke energie (het $I_{C} R_{N}$ product) door een niet-supergeleidende kanaal mogelijk te maken. De supergeleidende koppeling tussen de supergeleidende elektrodes vindt plaats op het oppervlak. De sterke koppeling naar het oppervlak is het resultaat van de anisotrope bandenstructuur in topologische isolatoren. De stroom/fase relatie in de juncties heeft het bestaan van een Majorana nulmodus nog niet aangetoond. De afwijkingen in het magnetische interferentie of Fraunhofer patroon in verschillende experimenten kan worden verklaard met fluxfocussering, defecten en de geometrie van de juncties op de oppervlaktes. 
Een gevoeliger onderzoek kan worden uitgevoerd met de Josephson junctie in een loepstructuur. Hiermee kan beter onderscheid gemaakt worden tussen in- en extrinsieke effecten die de stroom/fase relatie beïnvloeden.

Door de afstand tussen de elektrodes constant te houden en de breedte van de elektrodes te veranderen, wordt de lengte/breedte verhouding gevarieerd. Met deze aanpak is de invloed van het niet-supergeleidende kanaal en fluxfocussering onderzocht in juncties van $\mathrm{Nb}$ en $\mathrm{Bi}_{2} \mathrm{Te}_{3}$. Deze geometrische aanpak is voldoende om het gedrag in magneetveld en van de karakteristieke energie met variërende breedte te begrijpen. Het geschatte aantal normale of triviale modi in de juncties is in de orde van honderd. Het gevonden resultaat is dus niet verrassend: een mogelijke Majorana modus kan het gedrag van de juncties niet domineren.

Met het gebruik van een andere topologische isolator, namelijk $\mathrm{Bi}_{1.5} \mathrm{Sb}_{0.5} \mathrm{Te}_{1.7} \mathrm{Se}_{1.3}$, wordt het probleem van de niet-supergeleidende kanalen aangepakt, wat wel aanwezig is in juncties van $\mathrm{Bi}_{2} \mathrm{Te}_{3}$. Dit materiaal heeft namelijk geen geleidende bulk. De vrije weglengte van de oppervlaktetoestanden is echter sterk afgenomen. Het Josephson gedrag van gerealiseerde juncties, met een kritische stroom in de orde van IonA, wordt aangetoond door het meten van een Fraunhofer patroon bij $30 \mathrm{mK}$. Het Fraunhofer patroon wordt gekenmerkt door sterk onderdrukte oscillaties en periodes van enkele $\Phi_{0}$. Dit wordt verklaard door gebruik te maken van een model waarin transport met verstrooiing en juncties met open randen zijn opgenomen.

De stroom/fase relatie van juncties kan bepaald worden door de juncties op te nemen in een loep of SQUID. Met $\mathrm{Bi}_{2} \mathrm{Te}_{3}$ als de topologische isolator in een Josephson junctie is het conventionele gedrag van de juncties aangetoond. De oscillaties zijn namelijk periodiek in $\Phi_{0}$ in plaats van $2 \Phi_{0}$, en de gereduceerde modulatiediepte blijkt het gevolg van de inductie van de loep te zijn. Modellen van verschillende loepstructuren laten zien dat niet de periodiciteit maar de modulatiediepte van een SQUID aanwijzingen geeft voor een veranderde stroom/fase relatie. Een modulatiediepte kleiner dan men zou verwachten op basis van de inductie van de loep, is een aanwijzing van een $\Phi_{0} / 2$ stroom/fase relatie van de juncties. De gereduceerde modulatiediepte is afhankelijk van de verhouding tussen het aantal $\Phi_{0} / 2$ en $\Phi_{0}$ kanalen. 
Het tweede thema van dit proefschrift is het gedrag van roosters van Josephson juncties in een magneetveld. Een vierkant rooster van $300 \times 300$ niobium eilanden, $220 \mathrm{~nm}$ diameter en $45 \mathrm{~nm}$ dik, is op een gouden oppervlak gegroeid met een periode van $267 \mathrm{~nm}$. Nadat het systeem onder de supergeleidende transitietemperatuur afgekoeld is, ontstaat er een zogenaamd eierdoospotentiaal voor de excitaties van het systeem, namelijk de "flux vortices". Dit resulteert in een vortex Mott isolator. Een aangelegd magneetveld bepaald het aantal vortices en de frustratie in het rooster, terwijl een stroom een kracht uitoefent op de vortices. Voor geheeltallige frustratie, oftewel één vortex per kuiltje van de eierdoospotentiaal, vindt er een faseovergang plaats. Voor halftallige frustratie vindt er een faseovergang plaats met andere kritische exponenten. Er bestaat een afbeelding tussen deze vortex Mott isolator en een elektronische Mott isolator, waardoor het gedrag als functie van stroom en veld begrepen kan worden in termen van potentiaal en dotering.

Als vervolgens eilanden uit het rooster worden verwijderd wordt de eierdoospotentiaal verstoord. Translatiesymmetrie is nodig om oplossingen te kunnen vinden die het gedrag van het rooster als functie van magneetveld kan beschrijven. Er blijven echter kleine clusters eilanden over die voor symmetrie op een lokale schaal zorgen. Dit zorgt voor oscillaties in magneetveld die doen denken aan het gedrag van een gaaf rooster. De percolatiegrens, bepaald door het verband tussen de transitietemperatuur en de vulling van het model met eilanden, is lager dan voor een rooster waar alleen interacties tussen naaste buureilanden worden meegenomen. Dit is het gevolg van de Josephson koppeling tussen verder gelegen eilanden: de verwijderde eilanden onthullen dat er een merkbare koppeling tussen de een-na-naaste buren bestaat. 



\section{DANKWOORD}

Een proefschrift vormt niet alleen de afsluiting van ruim vier jaar onderzoek, maar vooral een afscheid van een groep mensen die allemaal, op hun eigen manier, ervoor gezorgd hebben dat ik die vier jaar door ben gekomen. Uiteraard ben ik iedereen dankbaar die ervoor gezorgd heeft dat de randvoorwaarden voor vier jaar werk klopten. In het bijzonder wil ik iedereen bedanken die mij heeft laten lachen tijdens het werk die afgelopen jaren:

Als eerste wil ik een tweetal duo's bedanken die voor continuïteit qua sfeer in de groep zorgde: Alexander \& Hans en Frank \& Dick. Denk aan de jaarlijkse zeil- en schaatsuitjes, de reis naar Engeland, het bewaken van momenten zoals het bejaardenfruithapje en de fietstochten langs alle ijstentjes van Twente. Bedankt voor jullie geduld, uitleg en aanmoediging binnen en buiten het lab. Ik hoop dat jullie het kunnen stellen zonder de koffie-aio!

Binnen de vakgroep ICE is mijn promotiecarrière bijna begonnen als vacuümleidinginstallateur met twee vaardige compagnons, Diederik \& Marcel. Daarna heb ik erg fijn samengewerkt met M \& M: Menno \& Marieke. Jullie enthousiasme was altijd aanstekelijk! Talking about enthusiasm, thank you Nicola, for your stream of ideas, and Francesco, from trying to protect me from them. I enjoyed the postdoc quest by you and Martin for a name for 王骁/任骁/Renshaw! I wish you guys all the best!

In meer dan vier jaar heb ik heel wat kamergenoten versleten. Jullie zou ik graag allemaal bedanken voor de gezelligheid en de gesprekken die over van alles en nog wat konden gaan: Kris, Joost, Thijs, Sam, Roel, Julius, Sébastian, Joris \& Bob. Ook de rest van de vakgroep daarvoor hartelijk dank!

De altijd verwarrende E-/IMS vakgroepen stonden niet alleen altijd vooraan als het om borrels ging, maar ik kon ook bij jullie terecht voor van alles wat ik nodig had qua apparatuur. In het bijzonder ben ik erg blij dat ik bij de vakgroep NE hun mengkoeler kon gebruiken en de geduldige expertise, especially from Elia!

Twee duo's die op de achtergrond mijn leven makkelijker gemaakt hebben zijn Harry \& Jan en Ans \& Inke. Het ene duo deed mij vaak denken aan twee sheriffs die met een getrokken 
pistool de schrik waren van experimenterende aio's, terwijl bij het andere duo vaak het grote boek van Sinterklaas op tafel kwam waarin mijn wegen werden bijgehouden. Vul zelf maar in wie welk duo is!

Buiten de muren van de Carré ben ik natuurlijk blij geweest met de ondersteuning en afleiding van mijn familie en vrienden. Mijn familie ben ik dankbaar voor de interesse, feedback en rust die jullie boden. En mijn vrienden voor de bredere blik buiten de muren van de vakgroep.

Mijn twee paranimfen, Marcel \& Joost, hebben qua ondersteuning en afleiding een belangrijke rol gespeeld op de universiteit én daarbuiten. Ik hoop nog veel alpencollen en bergtoppen met jullie te bedwingen, waarschijnlijk in het laatste wiel of uitglijdend in de sneeuw in het zicht van de top.

Het thema van dit dankwoord is inmiddels duidelijk: duo's. Bedankt Denise, dat je met mij een duo wil zijn! 